\title{
Improving Virtual Reality Safety Precautions with Depth Sensing
}

\section{Author:}

Shaoyan Huang

\section{Senior Supervisor:}

Prof. Rob Lindeman

\section{Co-Supervisor:}

Dr. Huidong Bai 


\section{Abstract}

Virtual Reality (VR) technology provides a computer-generated three-dimensional environment, in which the user can interact with virtual objects in real time and walk naturally with the locomotion techniques. However, because of the fully immersive design of VR headsets, free walking with VR headsets may result in people colliding with surrounding obstacles and cause serious physical injury. Securing users' safety is a prerequisite for any VR experience no matter in which kinds of circumstances. To improve VR interaction safety, virtual safety walls textured with a grid, such as HTC Chaperone and Oculus Guardian, have been widely adopted by many VR companies to remind users of safe interaction boundaries. However, they cannot provide detailed geometrical information of the environment, and limit users to stay within a small interactive space.

In this thesis, we developed two Augmented Virtuality (AV) interfaces that integrate depth sensing of surroundings into the VR scene for safety precautions, 1) a 3D Virtual Monochrome Grid (3DVMG), and 2) a 3D Virtual Coloured Grid (3DVCG). For 3DVCG, colours vary according to the distance objects are away from the user. We then conducted a user study to evaluate the usability of our proposed interfaces, and to investigate whether VR interaction safety can be improved with depth sensing and colour cues while the user navigates in VR. 2D Outlines of Surroundings (2DOoS) was used as a control.

There are two main findings from the experiment. First, with either of 3D interfaces, participants can navigate the virtual environment more confidently. Second, the colour visualization provides more hints to users for determining distances between real objects and their own positions and provide better assistance in avoiding obstructions than only superimposing the depth clues. 


\section{Acknowledgements}

This thesis would not have been possible without the support and guidance of my senior supervisor Prof. Rob Lindeman and co-supervisor Dr. Huidong Bai. Their advice and suggestions are precious in leading me to solve problems and conduct this research. I am also grateful for the assistance and encouragement of HIT Lab NZ students and staff especially Alaeddin Nassani, Yuanjie Wu, Humayun Khan, Harish Mandalika and Sungchul Jung through a difficult time. Lastly, I would like to thank my family for their constant caring and support. 


\section{List of Contents}

Chapter 1 Introduction

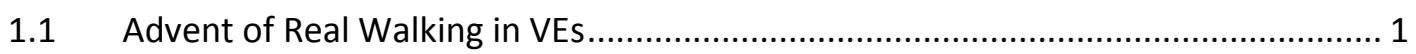

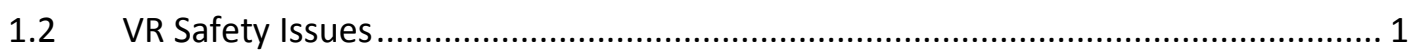

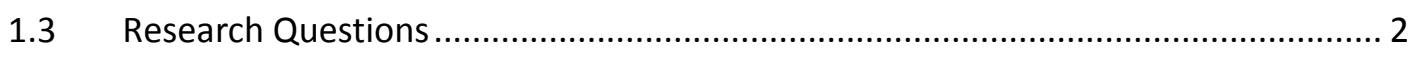

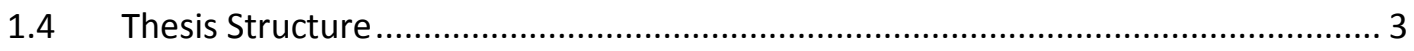

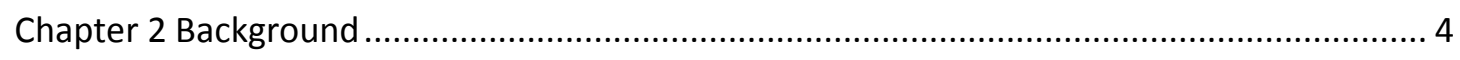

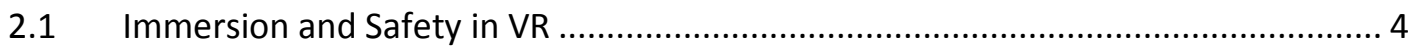

2.2 Immersion Regarding Walking ................................................................... 4

2.2.1 Increasing Sense of Presence with Natural Walking in VR ............................... 4

2.2.2 Assisting Users to Navigate in VR by Physically Walking ................................. 4

2.3 Importance of Safety Interfaces in VR ............................................................ 5

2.4 Implementation of Virtual Safety Interfaces ....................................................... 6

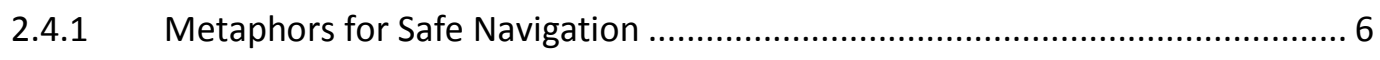

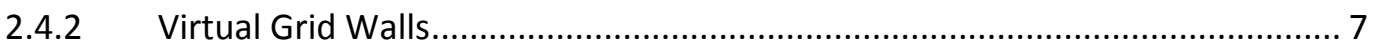

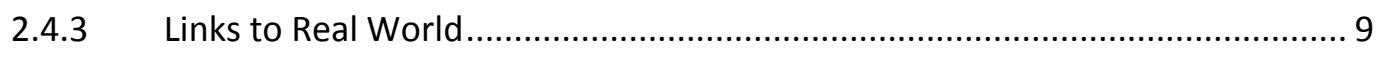

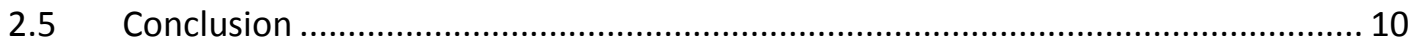

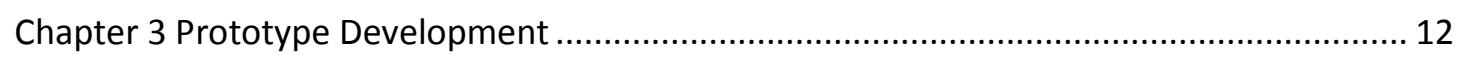

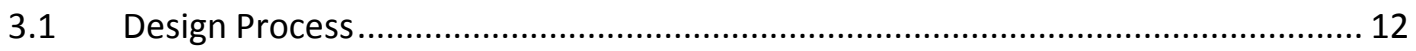




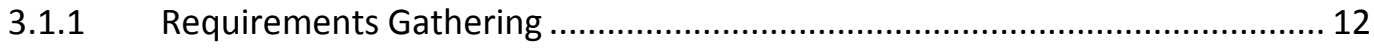

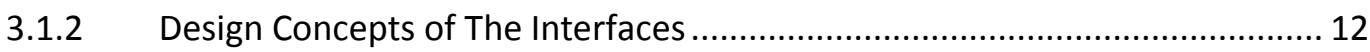

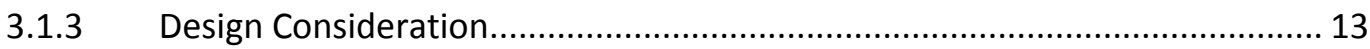

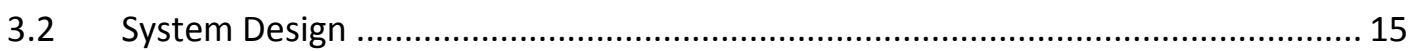

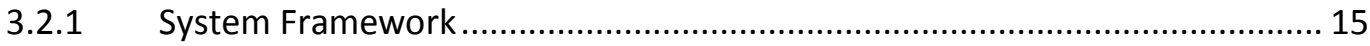

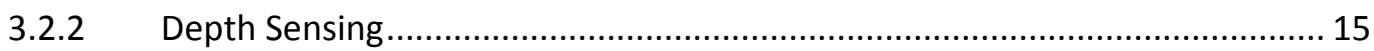

3.2.3 6 Degrees-of-Freedom Tracking .............................................................. 17

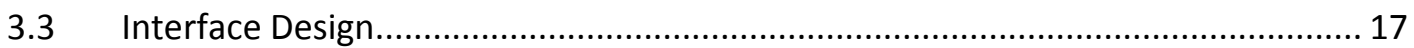

3.3.1 3D Spatial Mesh Reconstruction ……………........................................ 17

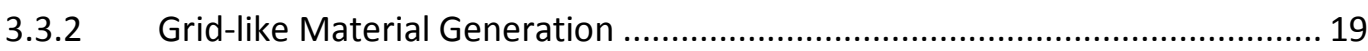

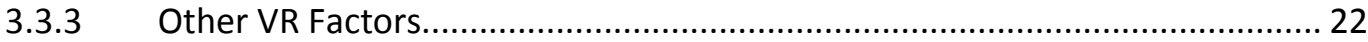

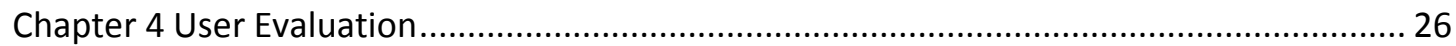

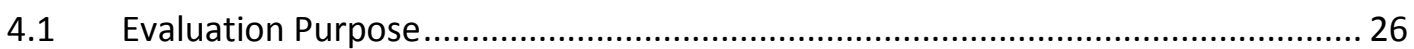

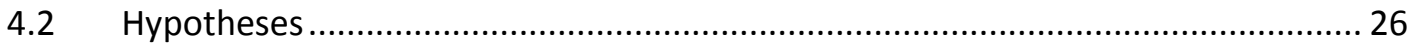

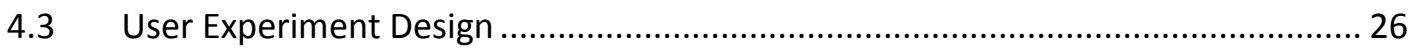

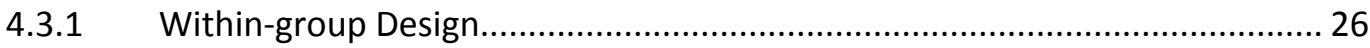

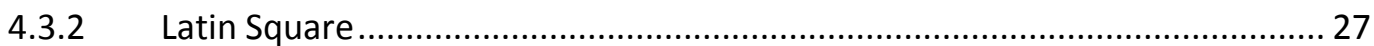

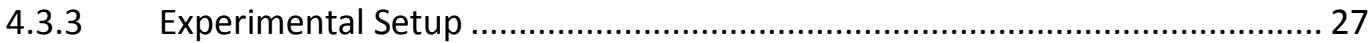

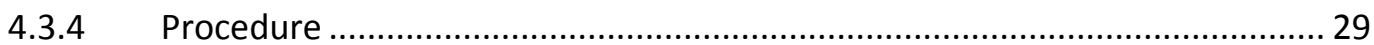




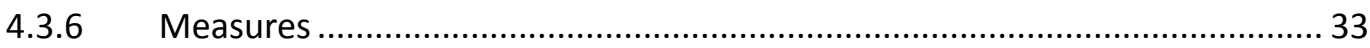

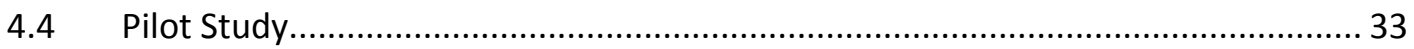

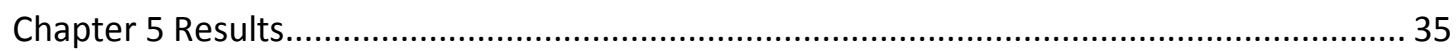

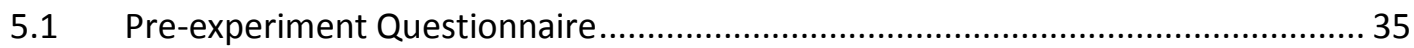

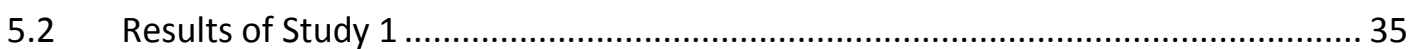

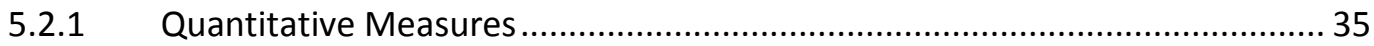

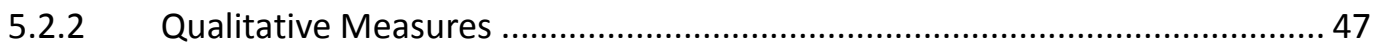

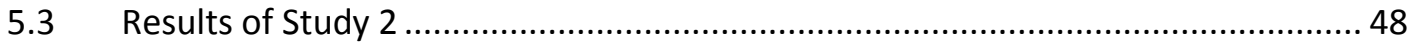

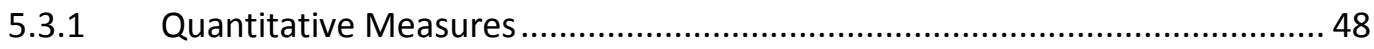

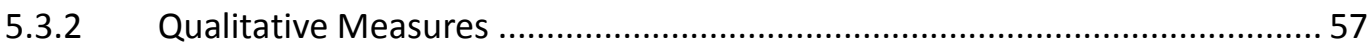

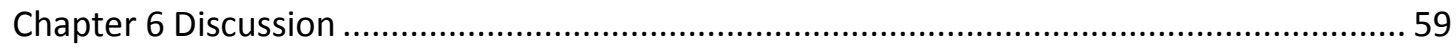

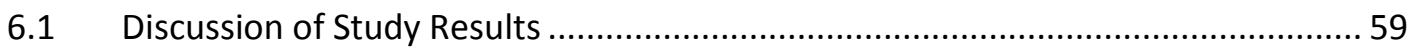

6.1.1 Users' Ability to Perceive the Obstacles in Study 1..................................... 59

6.1.2 Users' Ability to Perceive the Obstacles in Study 2 ......................................6 60

6.1.3 Comparison of The Usability of Two 3D Interfaces ...................................... 61

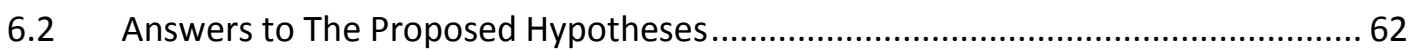

6.3 Answers to The Proposed Research Questions ................................................... 62

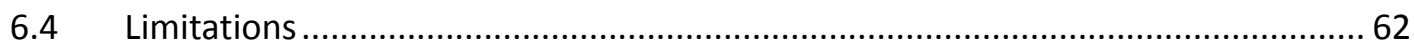




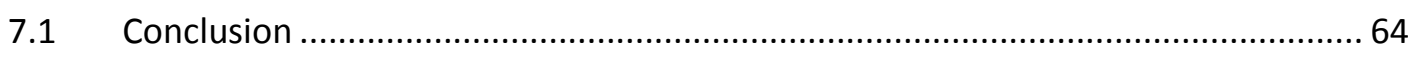

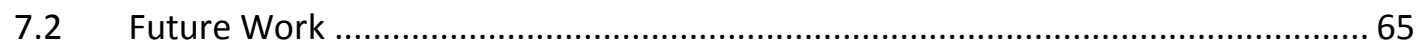

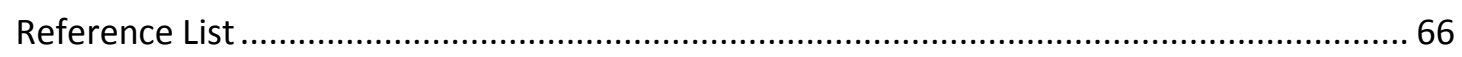

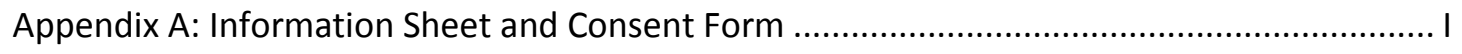

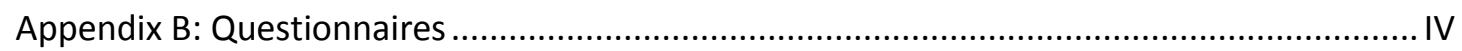




\section{List of Tables}

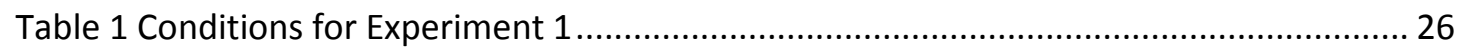

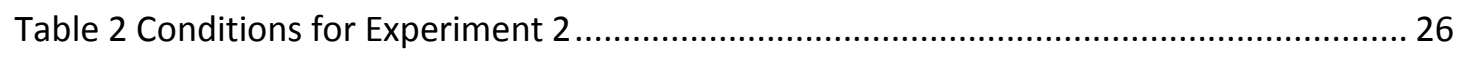

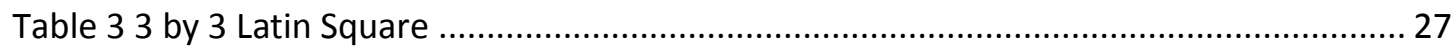

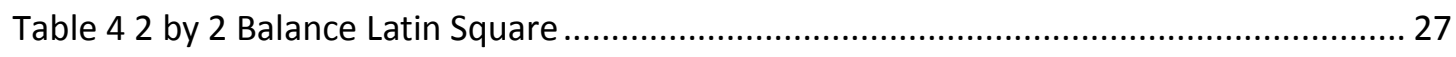

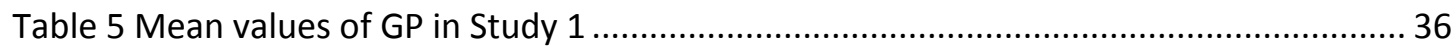

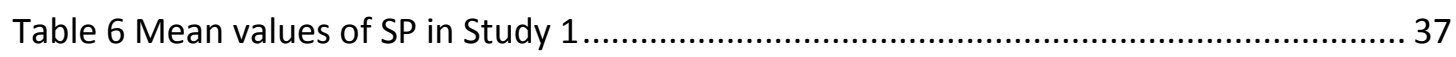

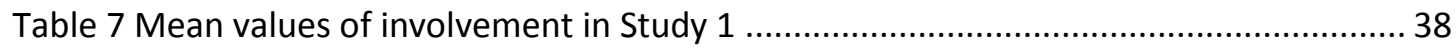

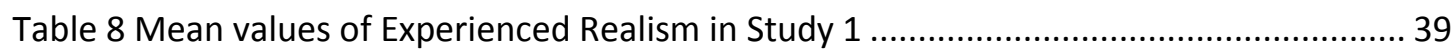

Table 9 Understanding of Real-space Information Questionnaire ....................................... 40

Table 10 Results of Understanding of Real-space Information in Study 1 ............................ 42

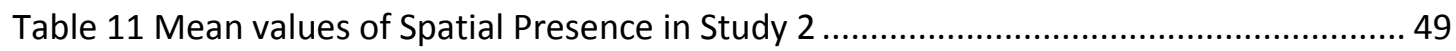

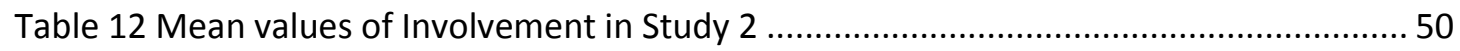

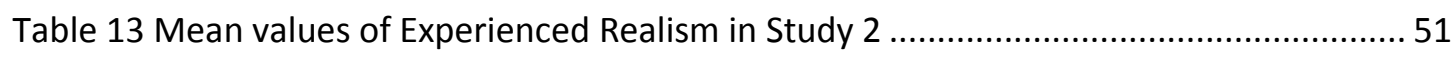

Table 14 Results of Understanding of Real-space information in Study 2 .......................... 53 


\section{List of Figures}

Figure 1 Setting virtual safety walls in the room-scale mode

Figure 2 Magic Barrier Tape [16]

6

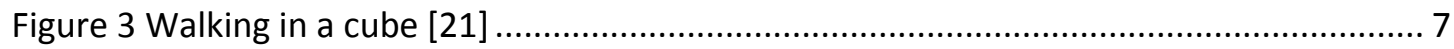

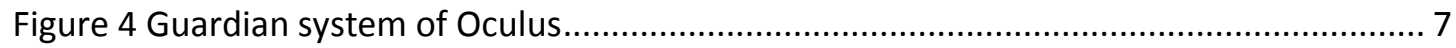

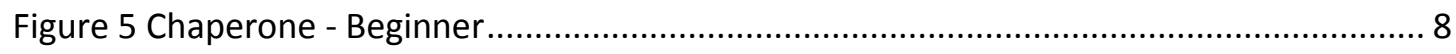

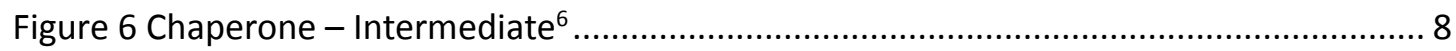

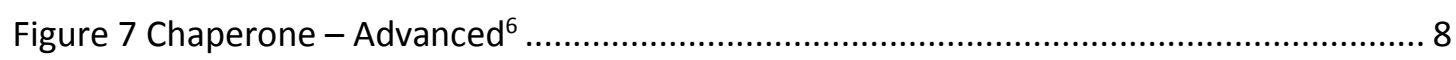

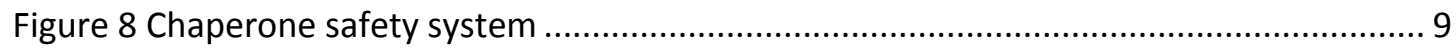

Figure 9 Tron effect, with a desk .......................................................................... 10

Figure 10 Tron effect, with a person ................................................................... 10

Figure 11 Augmented Virtual safety grids ................................................................. 12

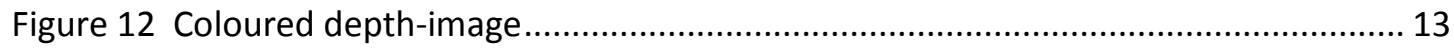

Figure 13 System framework ............................................................................ 15

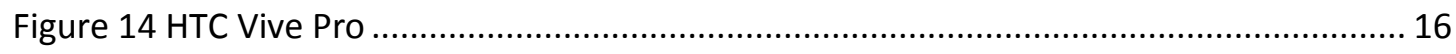

Figure 15 Spatial scanning as static meshes......................................................... 16

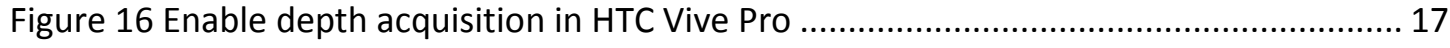

Figure 17 a) 3D Preview

b) Scan . 18 
Figure 18 Spatial scanning result.

Figure 19 a) Enable Mesh

b) Static (VR). 18

Figure 20 Loaded meshes 19

Figure 21 Target style of 3D grids. 19

Figure 22 Rendering workflow of Unity 20

Figure 23 Result of appending the new shader with one solid colour. 21

Figure 24 3D Coloured Grids based on different distance 21

Figure 25 3D Coloured Grids 22

Figure 26 Collision detection - Enter 23

Figure 27 Collision detection - Exit 23

Figure 28 Merge pieces into one mesh 24

Figure 29 Experiment 1 Setup 27

Figure 30 Experiment 2 Setup 28

Figure 31 Virtual Environment Setup ... 29

Figure 32 Study 1 Condition A - 2DOoS 30

Figure 33 Study1 Condition B - 3DVMG 31

Figure 34 Study1 Condition C - 3DVMG 31

Figure 35 Study 2 Condition A - 3DVMG 32

Figure 36 Study2 Condition B - 3DVCG. 32 
Figure 37 Results of GP in Study 1

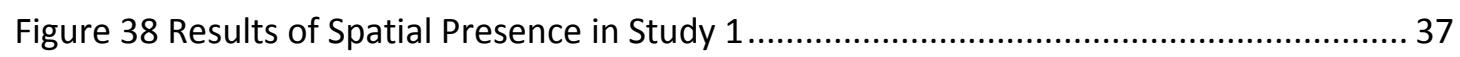

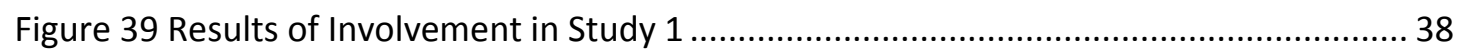

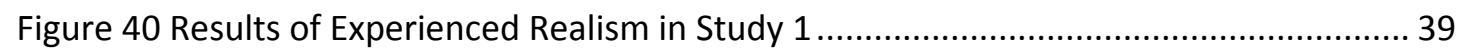

Figure 41 Results of Understanding of Real-space Information in Study 1 (Q1-Q8).............. 41

Figure 42 Results of Understanding of Real-space Information in Study 1 (Q9-Q13)............ 41

Figure 43 Results of Wilcoxon Signed Ranks Test ................................................................. 42

Figure 44 Ranking results of helping to predict the distance to physical objects .................. 43

Figure 45 Ranking results of insisting subjects to avoid obstructions ................................... 44

Figure 46 Ranking results of confidence of navigating the virtual space ............................. 44

Figure 47 Ranking results of overall preference............................................................... 44

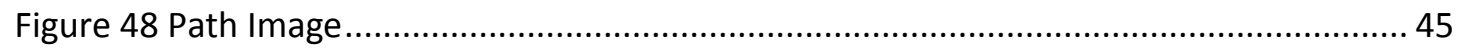

Figure 49 Results of Closest Distances to Chair in Study 1................................................ 46

Figure 50 Results of Closest Distances to Beanbag in Study 1 ........................................... 46

Figure 51 Results of Collision Times in Study 1 ............................................................. 47

Figure 52 Results of General Presence in Study 2 .............................................................. 48

Figure 53 Results of Spatial Presence in Study 2 ............................................................. 49

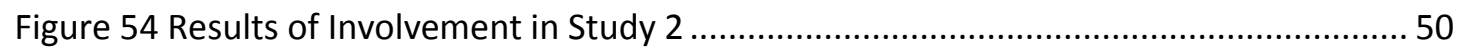

Figure 55 Results of Experienced Realism in Study 2 ..................................................... 51 
Figure 56 Results of Understanding of Real-space Information in Study 2 (Q1 -7)

Figure 57 Results of Understanding of Real-space Information in Study 2 (Q8 -13) 53

Figure 58 Ranking results of helping to predict the distance to physical objects 54

Figure 59 Ranking results of insisting subjects to avoid obstructions. 54

Figure 60 Ranking results of confidence of navigating the virtual space...... 55

Figure 61 Ranking results of overall preference. 55

Figure 62 Results of Closest Distances to Chair in Study 2 56

Figure 63 Results of Closest Distances to Beanbag in Study 2 56

Figure 64 Results of Collision Times in Study 1 57 


\section{Chapter 1 Introduction}

\subsection{Advent of Real Walking in VEs}

Virtual Reality (VR) technology provides a computer-generated three-dimensional environment. Users can interact with virtual objects inside Virtual Environments (VEs) in real time [2]. Recent results of a survey demonstrate that in the work and entertainment spaces, there is a trend of the resurgence of VR as a medium [22].

Typically, users are limited to sit or stand in front of a desk or table when they are playing VR games. However, this is not the only choice for current VR headsets. The latest devices produced by Oculus, HTC, and Samsung enable users to define their room-scale playing areas. Users can sit, stand or even walk in the pre-set areas with a 6 Degree of Freedom (DoF) tracking. Research has shown that compared with other locomotion techniques, real walking is preferred by users [24] because it is what people use in daily life [16].

Nonetheless, the sizes of VEs are normally larger than the real physical spaces. At the very beginning of development history of $\mathrm{VR}$, it was impossible for players to follow the synthetic paths to explore the endless virtual worlds because they ended up bumping into real walls. The introduction of redirection techniques [23] of VR has made a significant contribution to coping with this restriction of the real world. They allow players to break the boundaries of the physical environment to enjoy infinite spaces in virtual games by diverging virtual paths from physical movements [4] [23]. Furthermore, users can naturally walk and interact with content in the virtual space without breaking the illusion and compelling experiences of VEs [3]. Therefore, such locomotion techniques support users to immerse themselves in the synthetic world entirely and momentarily forget the real world [4].

\subsection{VR Safety Issues}

The strength of locomotion techniques becomes a weakness when considering safety issues though. People are blind and deaf to the real world while wearing VR headsets, and they have no clues or awareness of the locations of obstacles within interactive areas. Since they can freely walk, the possibility that they collide with real objects is higher than when sitting in a seat. In this case, virtual safety systems, such as warning grids [15], which can display boundaries of playing areas or offer glimpses of the real world to remind users of potential dangers are incredibly essential for players.

Several virtual safety metaphors have been proposed and implemented by researchers, such as the Magic Barrier Tape [16], Constrained Wands [21] and signs [21], Extended Magic Barrier Tape [21] and Virtual Companion [21]. They all have the functionality of preventing people from colliding with physical boundaries. However, their participants were only requested to walk a small distance, so more studies for large walking areas may have to be carried out. In addition, virtual safety walls textured with the grid are widely employed by VR products, such as the HTC Chaperone and Oculus Guardian. While users are in room-scale mode and they approach the predetermined boundaries, they can see the virtual safety walls from the VR scene (Figure 1). 


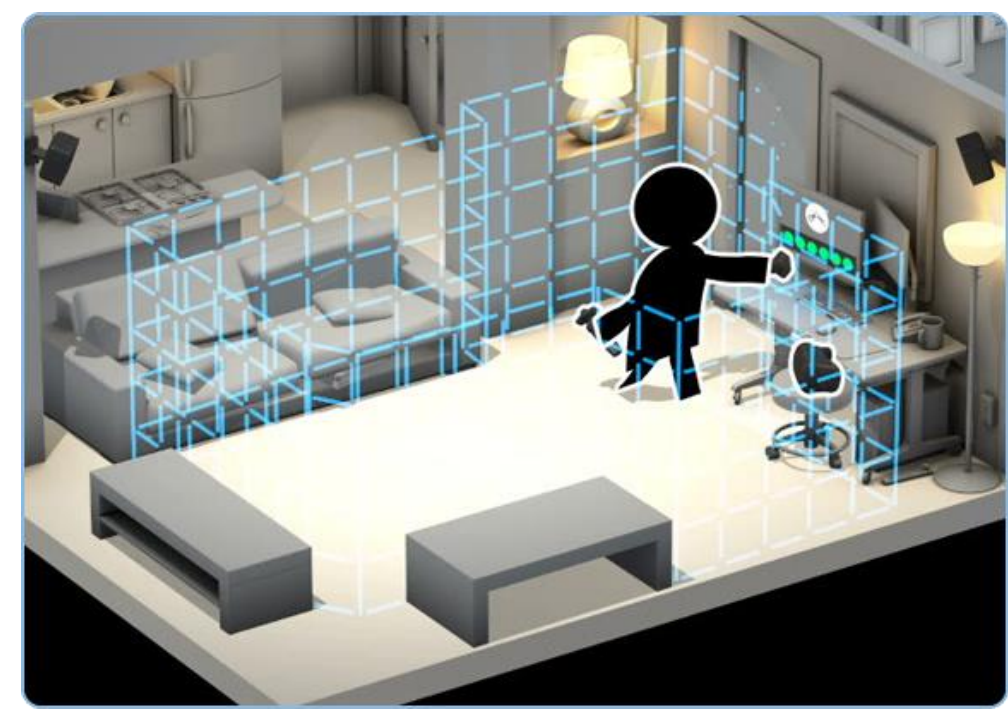

Figure 1 Setting virtual safety walls in the room-scale mode ${ }^{1}$

The warning grids are simple and less interruptive for users from an immersion perspective. However, they have their own limitations. For example, if more geometry information can be provided instead of only the vertical grids, users can make use of empty spaces above some obstructions such as tables in the VR space for more interaction. Advanced virtual safety interfaces (VSIs) demonstrating outlines or partial images of real-world objects for safely guiding users have been realized for the HTC Vive ${ }^{2}$ Compared with virtual grid walls, users can have a better understanding of their surroundings thanks to this safety system. The problem of this interface is its disruptive appearance, so there is a need to simplify its visual form. Overall, currently, existing virtual safety interfaces have their strengths and weaknesses from warning ability, visibility and immersion perspectives.

Another significant drawback of these virtual safety interfaces is that users must remove all furniture from the physical play areas to create an open space if they want to walk in VEs freely. Due to the reality that not all users can empty their rooms whenever they want to play VR games, and the popularity of mobile VR, there is an increasing requirement for improving safety precautions for users. In this thesis, we intend to develop 3D Augmented Virtuality (AV) interfaces that integrate the depth sensing of the surroundings into the VR scene to significantly improve a user's ability to perceive obstacles in the real world.

\subsection{Research Questions}

Improving virtual safety precautions requires furthering related research. In this thesis, we propose two AV interfaces which combine depth sensing of the physical environment into the VR scene, providing more detailed warnings for safely instructing users to avoid collisions while keeping a better interactive space.

This thesis will explore the following two questions:

\footnotetext{
${ }^{1}$ https://guides.library.unr.edu/4k/htcvive

2 https://www.vrheads.com/how-customize-htc-vives-chaperone-steamvr
} 
1. Can depth-based virtual safety interfaces improve interaction safety in VR environments for real walking navigation?

2. Can colour visualization based on obstacle depth improve interaction safety more?

In this thesis, two virtual safety interfaces blending the real world with VEs based on spatial scanning techniques will be implemented: Monochrome 3D Virtual Safety Grids, and Coloured 3D Virtual Safety Grids. For both interfaces, the whole real environment will be captured using a spatial scanning technique, and then this space will be textured with grids to offer shape information for users. For the first one, meshes will be rendered in one solid cyan colour, and for the second one, meshes will be tinted from red to blue according to their depth values, providing distance information and warning users.

\subsection{Thesis Structure}

The organization of the rest thesis is described here.

Chapter 2: Covers the background research focusing on immersion regarding walking in VEs, implementation of various virtual safety systems, and related work done on collision avoidance.

Chapter 3: Describes the design process and the implementation of the prototype.

Chapter 4: Describes the details of the user experiment and steps of the evaluation process.

Chapter 5: Presents the results obtained from the user experiment.

Chapter 6: Discusses the results found in the user study and the limitations found in this research.

Chapter 7: Concludes the thesis and identifies potential future areas of this research. 


\section{Chapter 2 Background}

\subsection{Immersion and Safety in VR}

"If you want to give people the most immersive experience to move around, they must be able also to move around safely. You cannot shoehorn that in later and hope it works well."

[Dan O'Brien, HTC $]^{3}$

Safety is key to immersion, and safety contributes to promoting immersive feelings in VR. They are complementary to each other. For example, it can be normal that people are involved in collisions with physical obstacles when they are too immersed in VEs without information of the real world around them. Conversely, if they slam into physical objects, a break-in-presence in VEs will be the consequence. These safety issues may not be obvious, while users only sit on chairs to play VR games. Nevertheless, real walking navigation in VEs has become a reality, and users prefer it to other virtual walking techniques due to increasing immersive experiences produced [24]. This results in the growing possibility of safety hazards. Here we study how to improve VR safety precautions with depth sensing. The literature review of this proposal focuses on immersive experiences resulting from locomotion techniques, the safety issues caused by them and existing implementations of VR safety interfaces.

\subsection{Immersion Regarding Walking}

\subsubsection{Increasing Sense of Presence with Natural Walking in VR}

It is well acknowledged that the sense of presence is one of the key determinants to evaluate the immersiveness of VEs. Regarding walking experiences in VR, the results of previous research have shown that compared with virtual walking and virtual locomotion implemented via joysticks and gamepads, exploring synthetic worlds through physically walking promotes subjects' sense of presence [4][5]. For example, real walking has advantages when subjects are asked to finish spatial orientation tasks over common joystick-based virtual travel techniques [8]. It has also led to a similar performance on search tasks [6] and has significantly increased players' awareness of avoiding collisions with virtual objects [7].

\subsubsection{Assisting Users to Navigate in VR by Physically Walking}

Physical walking motions cannot be directly mapped to virtual movement, because they result in the requirement of significantly large physical workspaces. Otherwise, the rate of the occurrence of players walking across boundaries and exiting tracking areas would be very high [4]. To satisfy the necessity of allowing users to travel long distances in VEs while they remain in limited real areas, several interface devices including torusshaped omnidirectional treadmills [10] [11], motion footpads, robot tiles [12] [13] and motion carpets [14] have

\footnotetext{
${ }^{3}$ https://www.engadget.com/2016/01/05/htc-vive-virtual-reality-chaperone/
} 
been developed. Unfortunately, weight, cost, safety and noise issues of these devices have hindered the general application of them.

Numerous redirection technologies have been introduced to overcome these limitations. Although navigating via walking, running or driving is easy in the real world, it has been difficult to duplicate the realistic simulation in VEs. Normally, in immersive VEs, movements of users' heads and walking in the real world are tracked and mapped to the virtual motion. With redirection techniques, movement within VEs can be different from that of the real world using adjusted translational and rotational gains to the user's motion, because the virtual camera is controlled by that. In a 2010 study, three experiments were conducted to estimate the detection thresholds of redirected walking techniques [9]. Results show that subjects' real motions such as rotating and walking are more or less than the perceived virtual motions, but users did not notice the difference [9]. This finding can be compelling evidence of supporting the functionality of redirection techniques in compensating for the lack of sizeable physical space.

Even though many desirable and promising results have been achieved in previous work, restrictions caused by limited physical space still exist. To reduce the severity of limited spaces, impossible spaces were introduced by Suma and his group in 2012. This is a novel virtual environment (VE) design mechanic aiming to maximise usable physical space in VEs, in which users can frequently travel around [4]. By combining existing redirection techniques with design principles of overlapping architecture, they produced vast synthetic worlds that players can naturally explore without concern about the real size of physical workspaces. They received positive feedback about the immersive qualities of the environment given by many participants after the experiments were finished.

In conclusion, these advanced techniques have mitigated the limitations of real physical spaces and provided large-scale immersive synthetic environments for users to freely explore. Such an ability to navigate through the virtual world with natural locomotion has resulted in increasing naturalness of VR-based interaction and more compelling experiences in many 3D applications, for instance, urban planning, tourism or 3D entertainment.

\subsection{Importance of Safety Interfaces in VR}

Guaranteeing physical safety is a prerequisite for any experience, no matter the circumstance, and considering how to protect people's physical health is a fundamental requirement for product design. At present, with developments of locomotion techniques mentioned above and consumer head-mounted displays (HMDs), users can have positive experiences with increased feelings of presence in VEs, but there are also significant safety issues, because users' connection to the real world is cut off [15]. When wearing a headset, their viewpoint is entirely blocked, and their intense feelings of presence in the VE take over. It is effortless for them to collide with real-world objects [15]. Reports of incidents are not rare. For example, while a user approaches the edge of the safe zone without visual presentation of real-world physical objects, they may punch or fall into real objects such a wall or their families or hit their head on the floor while looking through a virtual hole in the ground2. It is likely that such collisions can result in physical injury. Even if players do not hurt themselves or people around them, softly touching real objects can cause a break-in-presence as well [15]. In addition, since virtual spaces usually are larger than real workspaces, and the navigation techniques allow users to really walk to explore infinite virtual scenes, people easily and quickly reach boundaries. Dede argues that in a digital environment, it may be more dangerous for characters than in conventional environments [17], so it is more 
important to address safety issues in VEs [18]. Referring to the design of VR systems, comprehensive safety interfaces may be essential.

Several HMDs include room-scale tracking systems supporting players physically walk around a modest sized virtual space. In particular, with the HTC Vive ${ }^{4}$, greater mainstream access to this technology, the increasing possibility of injury or collision between users and stationary or moving obstacles can be foreseen, since exploring virtual environments by foot may become a norm for VR players. To address this problem, besides providing physical protection including harnesses, railings, or padding, fading in virtual safety walls is another widely used protection. Jerald claims that to provide visual cues to remind them of potential dangers, the design of fading in a warning grid or images of the real world in advance can minimize these problems [15]. Apart from that, various methods have been implemented by researchers to date.

\subsection{Implementation of Virtual Safety Interfaces}

\subsubsection{Metaphors for Safe Navigation}

To allow users to infinitely navigate the VEs within confined real spaces, a new interaction metaphor called Magic Barrier Tape was created by Cirio and his team [16]. There are two fundamental objectives of their design. The first one is informing users of the boundaries of physical areas, so they will not leave the tracking areas or collide with real objects. The second one is offering the possibility of reaching any location in the VE via an integrated navigation technique. When users move towards the edge of the tracking area, they will be exposed to the barrier tape (Figure 2), consisting of the main virtual barrier tape (middle), the warning virtual barrier tape (top), and users' grey shadow on the floor (bottom). Because it is decorated with black and yellow stripes, which is similar to what people can see in real life emergency scenarios, it presents the implicit message of "Do not cross!" to users. Researchers state that the Magic Barrier Tape can be implemented in various VR systems without the limitation of specific technology [16].

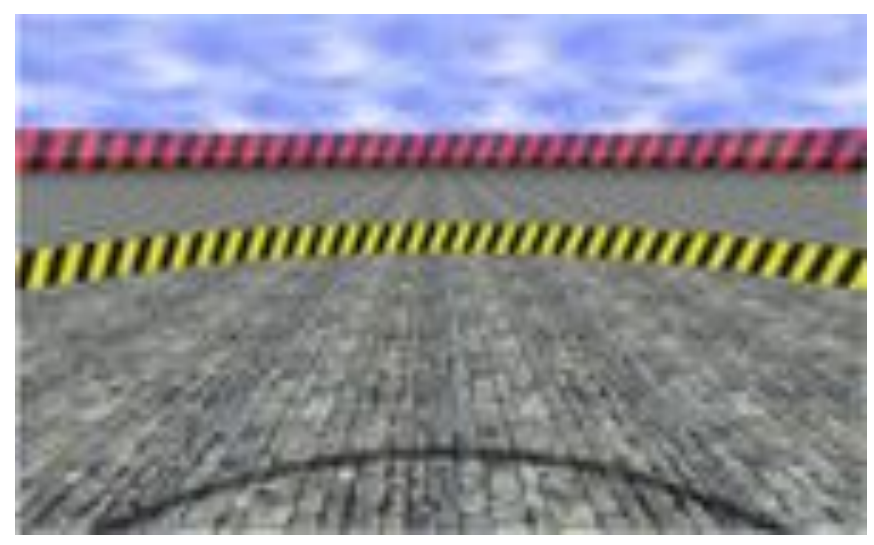

Figure 2 Magic Barrier Tape [16]

Based on this work, the researchers created extended Magic Barrier Tape (eMBT), which contains another three metaphors for guiding users to avoid reaching the translational and rotational boundaries in VEs [21]. The first

${ }^{4}$ https://www.vive.com/ca/ 
one is Constrained Wands and Signs (Figure 3a). Semi-transparent signs show up when the distance between users and boundaries are equal to specific values, and they will be transformed to fully opaque when necessary. The working mode of eMBT is similar to its predecessor, but the width of the tape changes until it can entirely block the users' viewpoint (Figure 3b). Regarding Virtual Companion (Figure 3c \& d), usually, a blue bird flies near the walls when users stay in safety areas. When users reach dangerous areas, the bird turns red and instantly flies in the face of the user to warn them. Results of this study have shown that subjects never collided with walls, and participants commented that eMBT is more useful than the other two metaphors in terms of warning them the real boundaries.
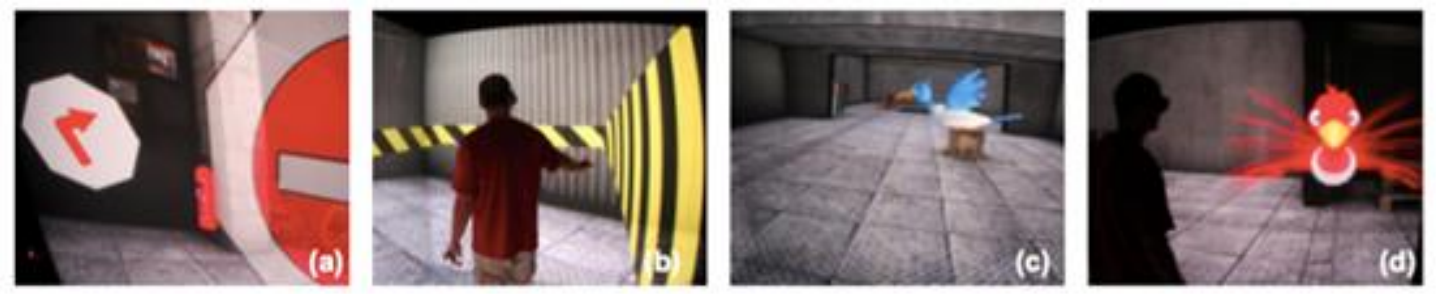

Figure 3 Walking in a cube [21]

However, their results also indicated that the currently used textures of the barrier tape reduce the visibility of virtual scenes when the dominant colour in VEs is similar to the tape colour. In other words, apart from reducing immersion in VEs, maintaining visibility is also very necessary for designing virtual safety walls.

\subsubsection{Virtual Grid Walls}

Grid walls are widely and most commonly implemented by various VR companies as their default safety systems, thanks to the simple style and basic functionality of informing users of boundaries of playing areas with little break of presence.

\section{a) Guardian}

Guardian is the boundary system developed by Oculus. During the setup process, users are required to set boundaries inside of which they can freely enjoy virtual games. When players get too close to the predetermined boundaries during the interaction, they see a warning grid indicating the edge of tracking (Figure 4).

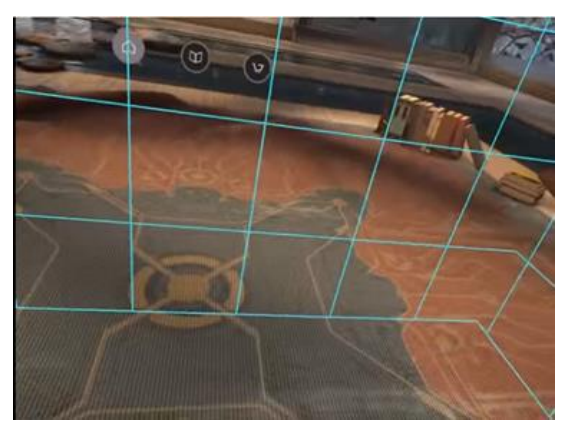

Figure 4 Guardian system of Oculus ${ }^{5}$

\footnotetext{
${ }^{5}$ https://www.windowscentral.com/how-customize-your-guardian-within-oculus-rift
} 
b) Chaperone

HTC produced its safety system for its players named Chaperone. In its safety system, there are three grid styles provided, including Beginner (Figure 5), intermediate (Figure 6) and Advanced (Figure 7).

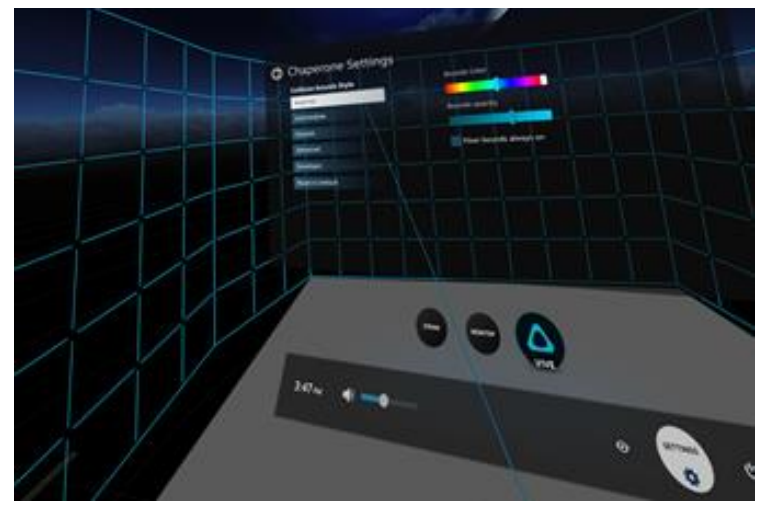

Figure 5 Chaperone - Beginner ${ }^{6}$

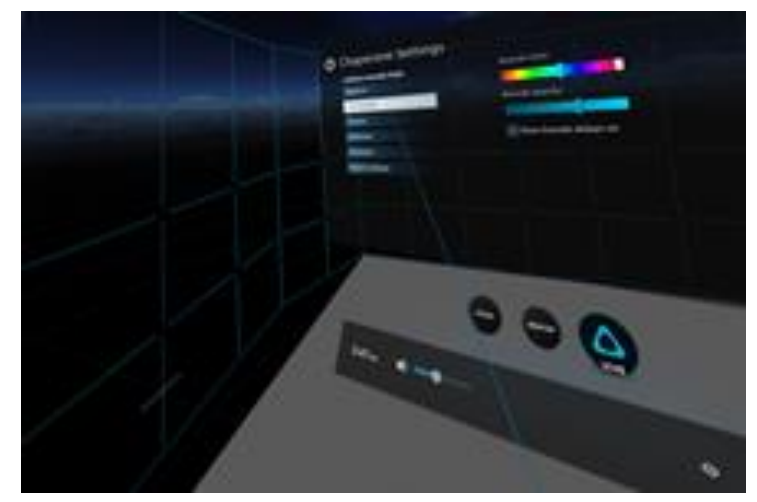

Figure 6 Chaperone - Intermediate ${ }^{6}$

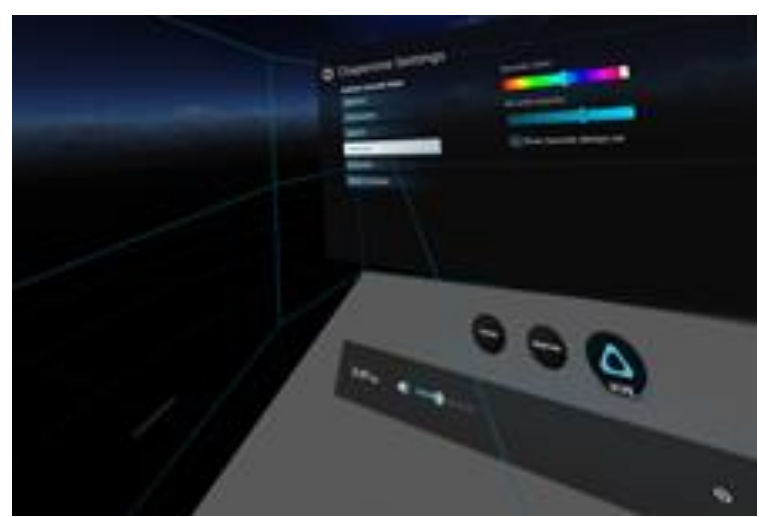

Figure 7 Chaperone - Advanced ${ }^{6}$

However, the strength of grid walls can be a weak point as well. First of all, they cannot display the information of objects outside boundaries, which can be useful for users in some cases. For example, when players are using their hands to finish shooting tasks in VR games, actually they can make use of the space above tables. As

\footnotetext{
${ }^{6}$ https://www.digitaltrends.com/virtual-reality/htc-vive-tips-and-tricks/5/
} 
anecdotal evidence, Eddie Lee from Funktronic Labs reported that when a tall tester was hitting balloons in VR, the controller accidentally beat the real-life ceiling light ${ }^{7}$ because there was no virtual wall showing the height of the ceiling. For some users, the style of grid walls is unobvious, and they may forget it while being too focused in the VE. A Wobbly Duck Studios developer reported that he could not distinguish the virtual walls from real ones and ended up slamming into solid walls ${ }^{5}$.

\subsubsection{Links to Real World}

HTC also offers an opportunity for its consumers to customize their safety interfaces via activating the frontfacing camera. It blends partial reality with VEs for providing more details to remind users.

a) Mixed Reality

As shown in Figure 8, when the player leans towards a table, a part of the table is projected into the VE. This is a feature of the HTC Chaperone system, which matches with concepts of design principles of safety proposed by Jerald [15], exposing users to Mixed Reality (MR). MR is an environment that facilitates users to simultaneously see other players or objects in the real world and the virtual environment [19]. This design allows users to recognize real objects quickly, and then they can perform some necessary interactions. For instance, taking a drink. However, to minimize the interruption resulting from the presentation of real objects, the proportion of reality to show should be carefully taken into consideration. Researchers have investigated this problem and concluded that the optimal solution is presenting to users selected content of reality with which they intend to interact [20].

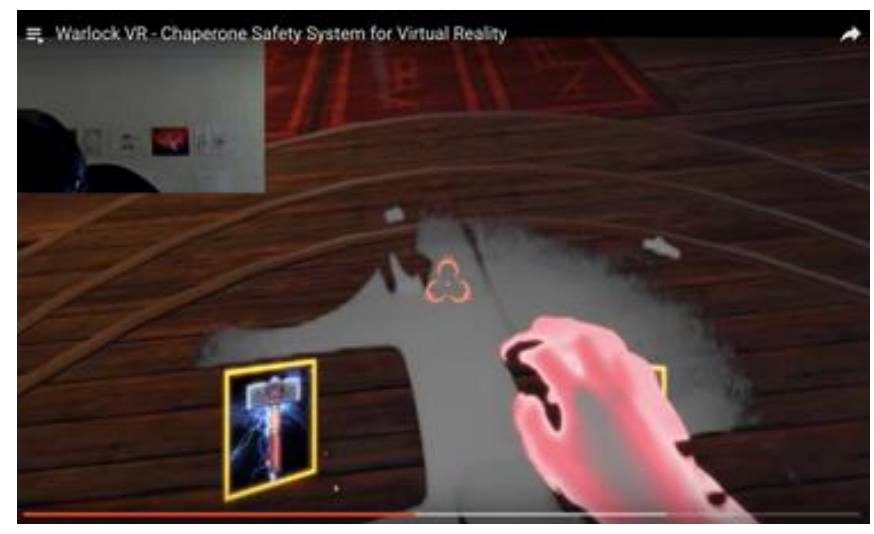

Figure 8 Chaperone safety system ${ }^{8}$

b) Outlines of Surrounding Objects - HTC Tron Mode

In Tron Mode, when players approach the pre-set boundaries, they can see the default safety walls and twodimensional edge-based presentations of their real environments (Figures $9 \& 10$ ).

7 http://www.businessinsider.com/virtual-reality-gamers-are-literally-colliding-with-the-physical-world-20164 ? IR=T

${ }^{8}$ https://www.youtube.com/watch?v=v5qcjJbBwA0\&list=LLn52khUOSiPRAVpoU6xTHzw\&index=3\&t=0s 


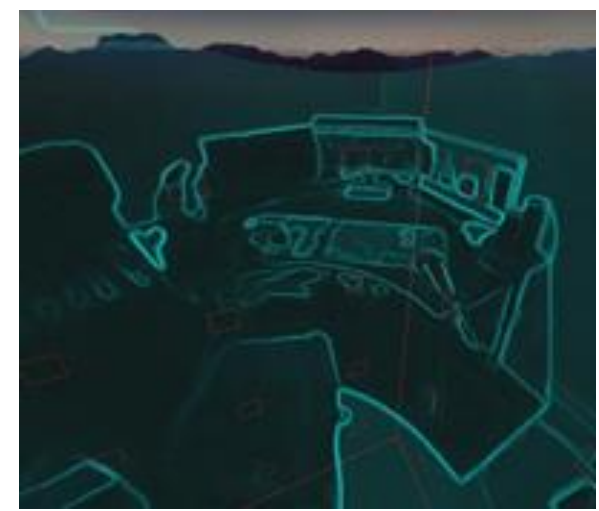

Figure 9 Tron effect, with a desk ${ }^{9}$

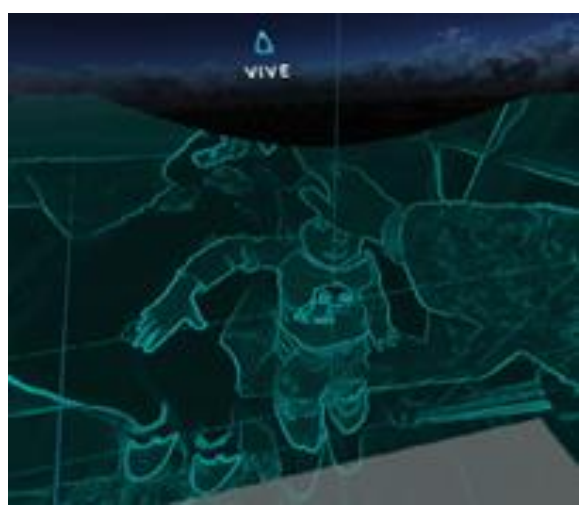

Figure 10 Tron effect, with a person ${ }^{10}$

With the assistance of this system, people can stop in front of a table and grab a drink when they feel thirsty, or they can quickly perceive their positions in the rooms and take action to avoid obstacles. However, the visual effects of this mode are undesirable. It can be difficult to distinguish the physical objects due to incomplete stroking and complex rendering styles. Also, as it presents the outlines of the entire workspace, it can be difficult for users to concentrate on virtual games due to such an interruptive background. Therefore, these limitations need to be addressed in terms of functionality and immersion experience.

\subsection{Conclusion}

Overall, there are three main categories of virtual safety systems, including metaphors, warning grids and links to the real world, and they have their own advantages and shortcomings. Metaphors of various signs which people can see in daily life have the capacity for conveying the same implicit messages to users, but visibility issues have not been well addressed yet. In terms of warning grids, they can show boundaries of playing areas to remind users of the possibility of collisions. However, there is a potential requirement of adjusting the appearance of the real world for users. For the MR virtual safety systems, players can perceive their locations better, but careful selection of the displayed information and visual style are needed to be thoughtfully

\footnotetext{
${ }^{9}$ https://www.vrheads.com/how-customize-htc-vives-chaperone-steamvr

${ }^{10}$ https://www.makeuseof.com/tag/virtual-reality-is-finally-here-htc-vive-review-giveaway/
} 
considered. Augmenting the virtual space by appropriately overlaying the real-space information can be an ideal way to guarantee players' safety without breaking their sense of being in the virtual world. Therefore, our research highlights the possibility of developing safer and more effective virtual safety interfaces with depthsensing techniques for providing detailed spatial information of the real space to users. Grids and colours will be mapped to physical spaces to display shapes of real objects, which allows users to quickly perceive the locations of obstructions and predict distances between themselves and real objects. 


\section{Chapter 3 Prototype Development}

\subsection{Design Process}

\subsubsection{Requirements Gathering}

Since our experiment was planned to be carried out under a walk-compatible circumstance, the HTC Vive Pro, a VR device that allows users to navigate through the VE via naturally walking was used in our study. Our VR testing would be in a room-scale playing mode, in which users can move freely within a pre-set area. It is well known that in the typical VR room-scale mode, an unobstructed space exclusively for VR is required, and the HTC Vive Pro is not an exception. The current strategy to protect players' safety equipped with the HTC Vive Pro is visualizing virtual safety walls or simple outlines of the real environment (Tron Mode) when people approach the boundary of playing areas. In our study, we were particularly interested in whether navigation safety can be improved via integrating depth sensing and depth-based colour visualization compared with the Tron Mode. To realize this, we used the latest HTC Vive Pro 's front dual cameras, which can capture depth information of the physical space in real time. Once the implementation of our interfaces was finished, we assessed the proposed methods for immersion and usability in providing precaution to users through user evaluation.

\subsubsection{Design Concepts of The Interfaces}

As mentioned in Chapter 2, the current virtual safety wall adopted widely by most VR companies can only remind the user of the boundary positions, and no geometrical information of surroundings is provided. An AV system built into the Bridge Engine developed by Occipital gives us an inspiration ${ }^{10}$. In their system, the real environment is scanned by the Structure Sensor and a digital reconstruction is consequently generated. The room's 3D data is used as a safety system to remind users of furniture and potential dangers (as shown below).

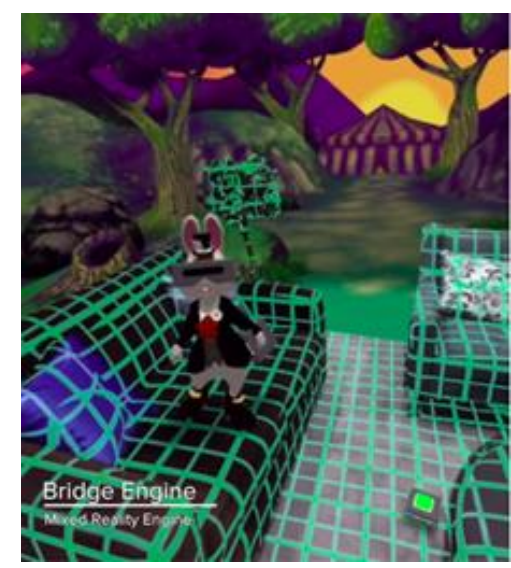

Figure 11 Augmented Virtual safety grids ${ }^{11}$

${ }^{11}$ https://www.tomshardware.co.uk/occipital-structure-mobile-6dof-tracking,news-53967.html 
However, no relevant user study for this system has been conducted to compare its advantages with other existing ones, and its monochrome style can also be improved to achieve better results with depth colours. Therefore, with our 3D interfaces, we intended to show more spatial information of the real space in VEs to users for safety precaution purposes, including geometrical and depth information. The initial plan of this research was to develop two different virtual safety interfaces by utilizing these techniques, namely 3D Virtual Monochrome Grid (3DVMG) and a 3D Virtual Coloured Grid (3DVCG).

a) 3D Virtual Monochrome Grid

The technique of generating grids with spatial information is spatial mapping supported in the HTC Vive SRWorks SDK ${ }^{12}$. After the grids of the entire environment within the play area were created, they were textured in a grid style in this variant. When users approach the pre-set boundaries, they are exposed to this safety interface.

b) 3D Virtual Coloured Grid

Studies have shown that the colour red is normally associated with danger and mistakes, such as stop signs and warnings [26], and can activate avoidance motivation [1] [25]. Therefore, in this safety interface, when users approach the edge of the available zone, we show red colour at first and then other parts of grids decorated with various colours. To achieve this, the outcome of the 3DVMG, which contains the depth data calculated by the SRWorks SDK, is used to implement a coloured virtual safety grid. To be specific, according to different depth values of physical objects, the grid is coloured from red to blue as in Figure 12, in which red for near elements indicates the distance between users and obstacles is very short and dangerous, and blue for far elements indicates objects in the real world which are in the distance and less threatening.

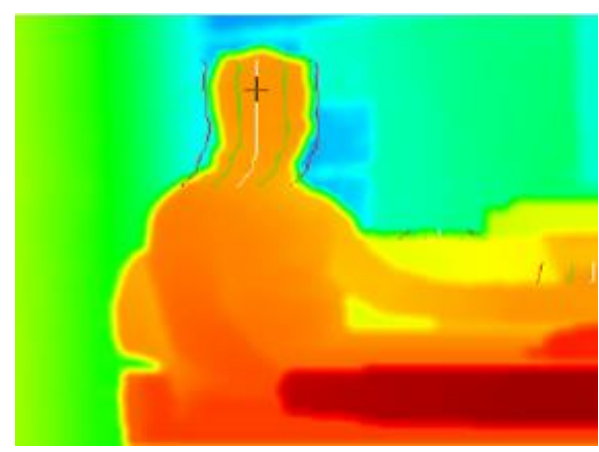

Figure 12 Coloured depth-image ${ }^{13}$

\subsubsection{Design Consideration}

The primary target is investigating the performance and usability of our novel VR safety interfaces in warning players of existing obstacles in the physical world under real walking navigation tasks. Firstly, we built a virtual space in which subjects could navigate via real walking by following fixed routes. During the exploring process,

\footnotetext{
12 http://community.viveport.com/t5/Developer-Blog/Announcing-VIVE-SDKs-for-the-Vive-Pro-front-facing-cameras-and/ba-p/17573

13 https://www.researchgate.net/figure/A-colored-depth-image-Near-elements-are-red-far-elements-are-blue-The-verticalgreen_fig1_263291984
} 
there is a possibility that they will collide with preplaced physical obstacles positioned in the way of routes. For the second stage, we generated two 3D grid-style virtual safety interfaces based on constructed meshes. The last step is loading interfaces into the created virtual space, and gradually visualizing the safety meshes whenever users are about to bump into obstacles in playing areas. We evaluated these two safety warning interfaces with a formal user study. We also recorded subjects' different avoidance behaviours in different testing conditions during the experiment, such as motion paths of players. The considerations of different parts are described as follows in detail.

- From the environmental setup perspective, the primary goal is providing fair chances that players will collide with real obstacles. Two main factors are considered, 1) how will the player navigate himself/herself in the virtual space and 2) where will real objects be placed. In this case, if subjects are allowed to walk randomly with no extra requirement, it would be difficult to guarantee the fairness of the potential that every subject will collide with real obstructions during the navigating process. Therefore, in the virtual space, several predetermined routes are rationally designed to match with the physical and virtual environments flawlessly. For example, ensuring that the routes suit to the size of real space, and subjects walk on appropriate paths in the virtual world instead of some unusual walking places, such as rocks or mid-air. Apart from that, virtual obstacles were removed in the way of the fixed routes as well. Otherwise, it would be uncertain whether players were avoiding virtual objects or real ones. In the testing physical space, there was some randomly positioned furniture, and the possibility that players would bump into the furniture is the same no matter which virtual route they take.

- When designing our two interfaces, one significant requirement was being able to scan the real environment and produce 3D meshes with depth information included, which can be implemented using the HTC Vive Pro. The warning performance of colours was another aspect we intended to assess. Therefore, both safety interfaces would include depth information of the real space, but they would be coloured in different styles, monochrome and multicoloured. Since the HTC Tron interface is to be used as a reference in our experiment, we select cyan for the monochrome colour which is currently used by HTC Tron. For the other one, it would need to be tinted in different colours according to the depth values of physical objects. For example, red, green and indigo can be used to indicate the near, median and far away distances respectively.

- In terms of demonstrating ways of proposed interfaces, to guarantee the equality of three interfaces in the further experiment, the ideal method could be simulating the one presently employed by the HTC Tron interface. For example, our new virtual interfaces would be gradually presented while players stayed in the dangerous zones by increasing or decreasing the transparency of grids according to the distances between players and obstacles as the HTC Tron does.

- The final element, which plays a crucial role in completing the development of the interfaces, was the approach to trigger the interfaces. At present, the HTC Tron only appears when players are close to the pre-set barriers. Nevertheless, we designed that the warning interfaces could be demonstrated to users for safety purposes without the limitation of their positions, it could be more compatible and flexible to more situations. For example, imagine that a player is in the middle of the playing area, if there is an obstruction, they are still capable of avoiding it due to the hints given by safety interfaces.

To summarize, construction of the virtual world, appearance design, displaying ways and triggering methods of the interfaces are essential factors, which should be taken into consideration for the implementation of this study. 


\subsection{System Design}

\subsubsection{System Framework}

The following picture demonstrates the framework of our prototype. The whole process of how the real environment is captured, customized and being displayed in the output (the HTC Vive Pro) is described in the Figure 13. Firstly, 3D meshes of the real environment are the input needed in our system. To realise this, we use the HTC Vive Pro to scan the real space and HTC ViveSR SDK to generate several corresponding meshes with geometrical information. During the visualization process, multiple 3D meshes acquired in the first step are joint into a single one, and then it is altered into a desired monochrome grid style or a coloured grid style using different shaders. Lastly, these virtual grids are augmented into the virtual environment which are outputted into the HTC Vive Pro headset.

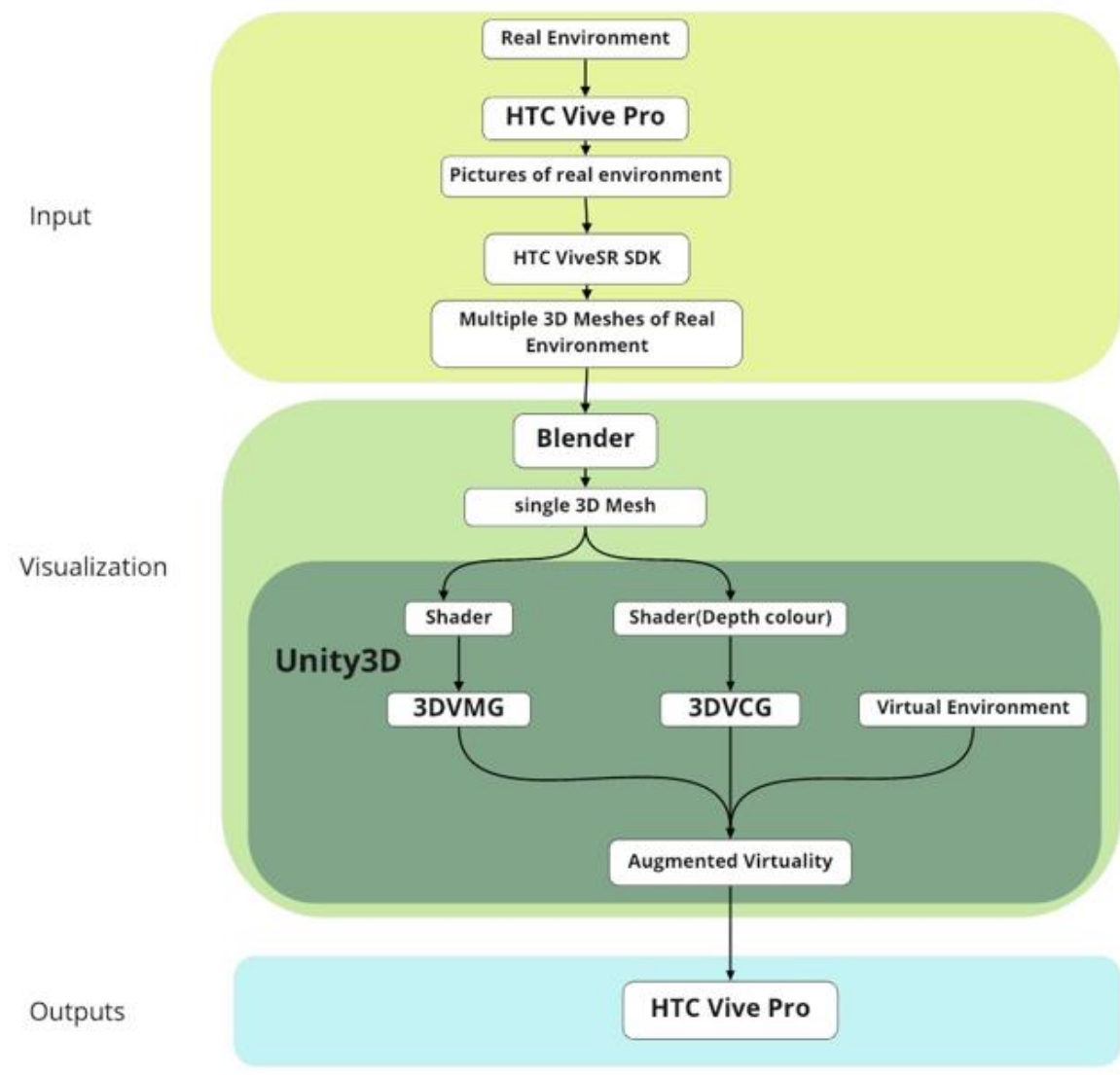

Figure 13 System framework

\subsubsection{Depth Sensing}

As mentioned before, the device chosen for this study is the HTC Vive Pro, which contains stereo cameras in front (Figure 14) and provides access to its spatial scanning and depth sensing features for developers. The two 3D Virtual Safety interfaces of this study were developed based on these resources. 


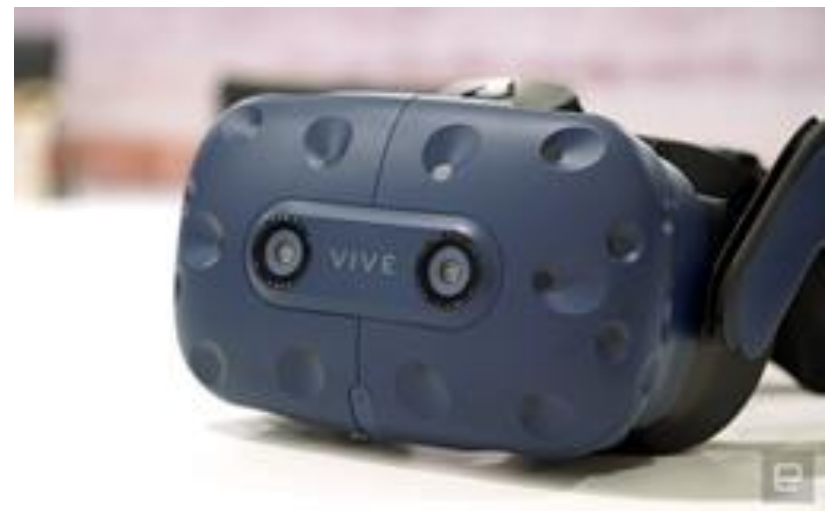

Figure 14 HTC Vive Pro ${ }^{14}$

With the spatial scanning feature, the HTC Vive Pro can scan the real environment and save them as static meshes (Figure 15). This technique was used for generating 3D meshes of real surroundings in our study.

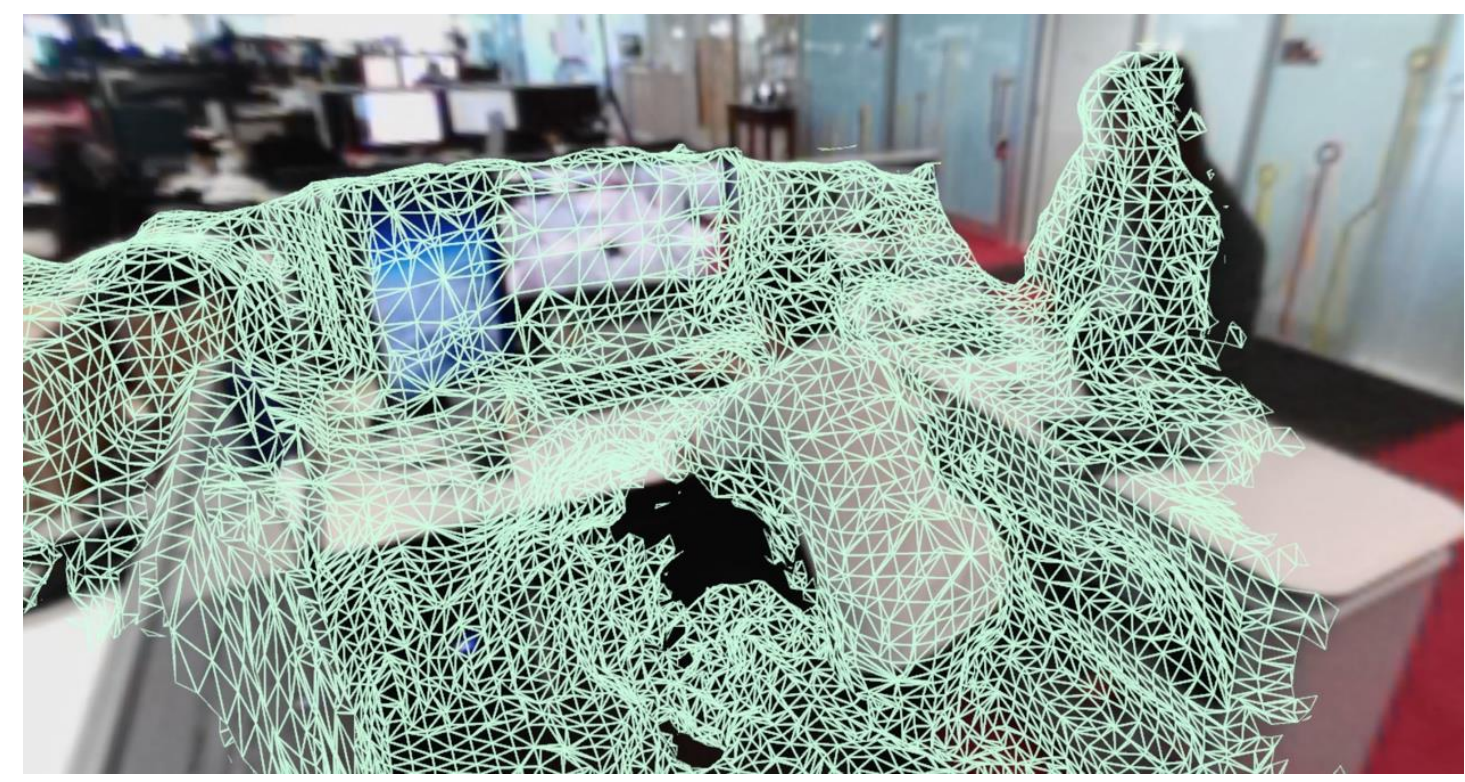

Figure 15 Spatial scanning as static meshes

Meanwhile, the depth values of physical space can be captured in each frame, and corresponding coloureddepth images (Figure 16) can be simultaneously generated. This technique was used for tinting the 3D coloured virtual safety grids.

${ }^{14}$ https://www.vive.com/nz/product/vive-pro/ 


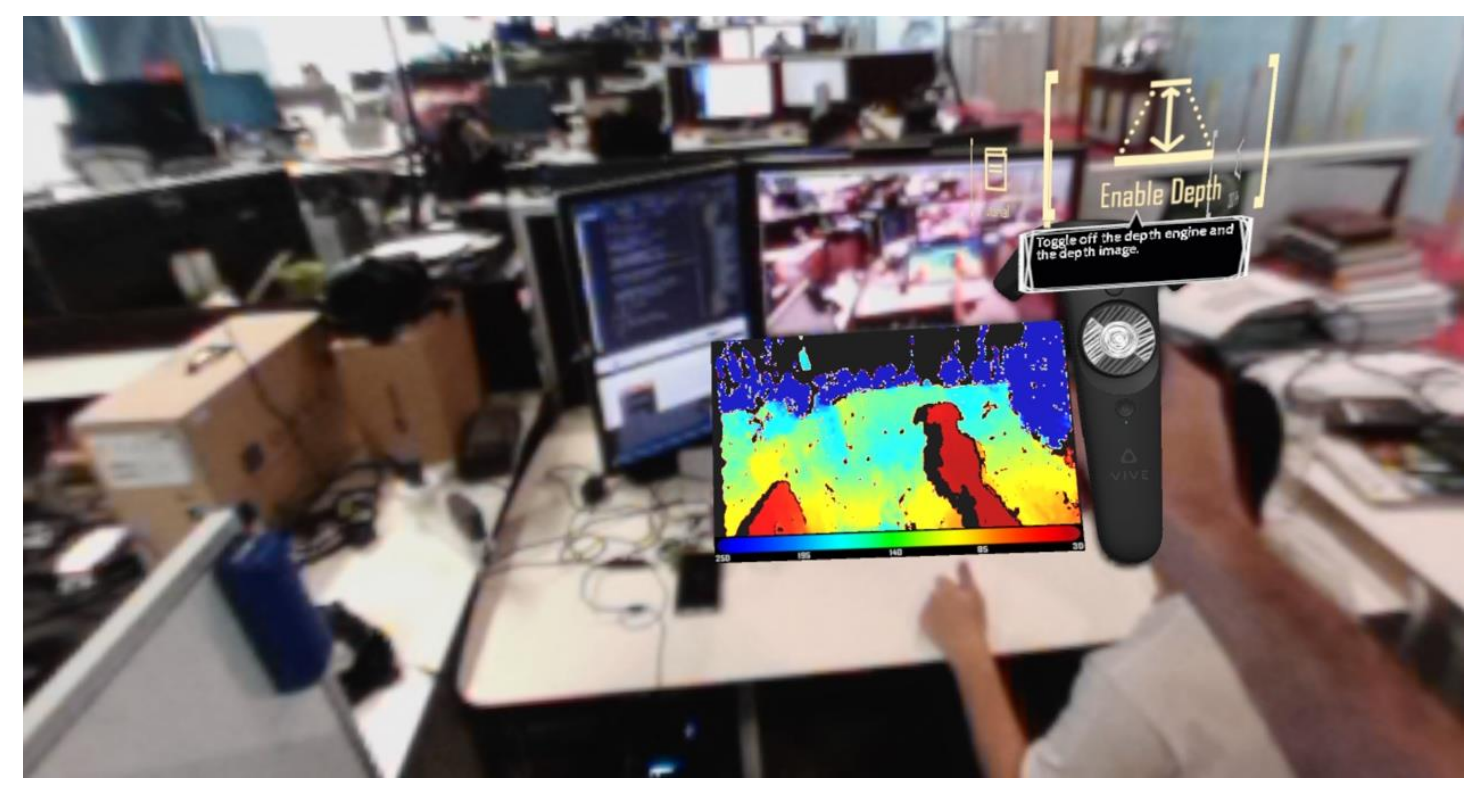

Figure 16 Enable depth acquisition in HTC Vive Pro

\subsubsection{Degrees-of-Freedom Tracking}

Since the focus of this thesis is to estimate the effectiveness of two novel virtual safety interfaces, participants would be encouraged to move around the workspace during the experiment freely. Therefore, the headset used in this thesis has the capacity for tracking their movements on a large scale. After connecting the HTC Vive Pro headset to a VR supported computer and setting up two base stations, the VR system can support precise 6 Degree-of-Freedom (6DOF) orientation and position tracking of the user's headset and hand-held controllers when they walk around the real room.

\subsection{Interface Design}

We used Unity, a professional platform that supports VR development, to construct virtual scenes and develop our interfaces, and to access the spatial scanning function provided in HTC Vive Pro, the HTC SRWorks SDK was imported for the coming implementation.

\subsubsection{D Spatial Mesh Reconstruction}

In this study, the interfaces are designed to present the spatial information to users for collision avoidance purposes, 3D spatial models of the real space are undoubtedly the determinant elements for interfaces construction. As mentioned before, the real space can be scanned and saved as 3D meshes automatically by using the HTC SRWorks SDK. Thus, the first step was running the application with the spatial scanning feature.

Once the application is working, six options can be found by rotating the controller. After selecting "3D Preview > Scan" (Figure $17 \mathrm{a}$ and b), the front stereo cameras of the VR headset will start capturing the frames and calculate the spatial information and then generate the 3D grids, as shown in Figure 18. 


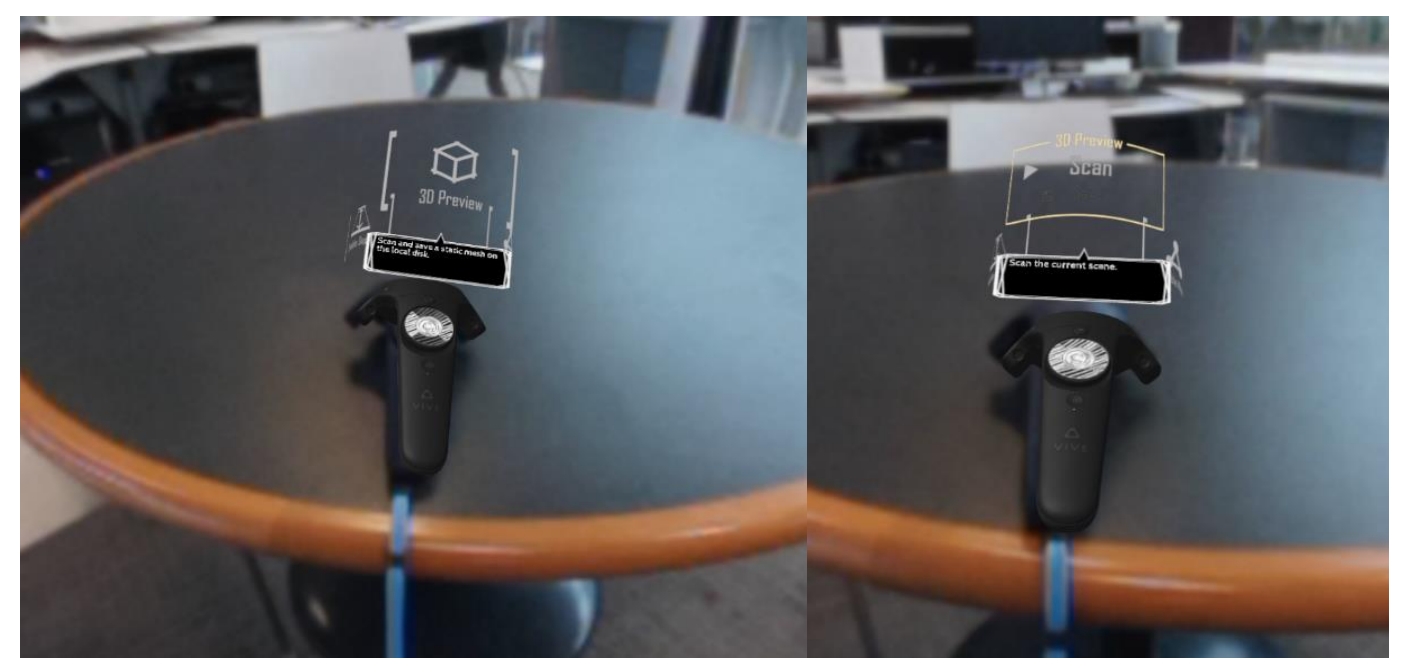

Figure 17 a) 3D Preview

b) Scan

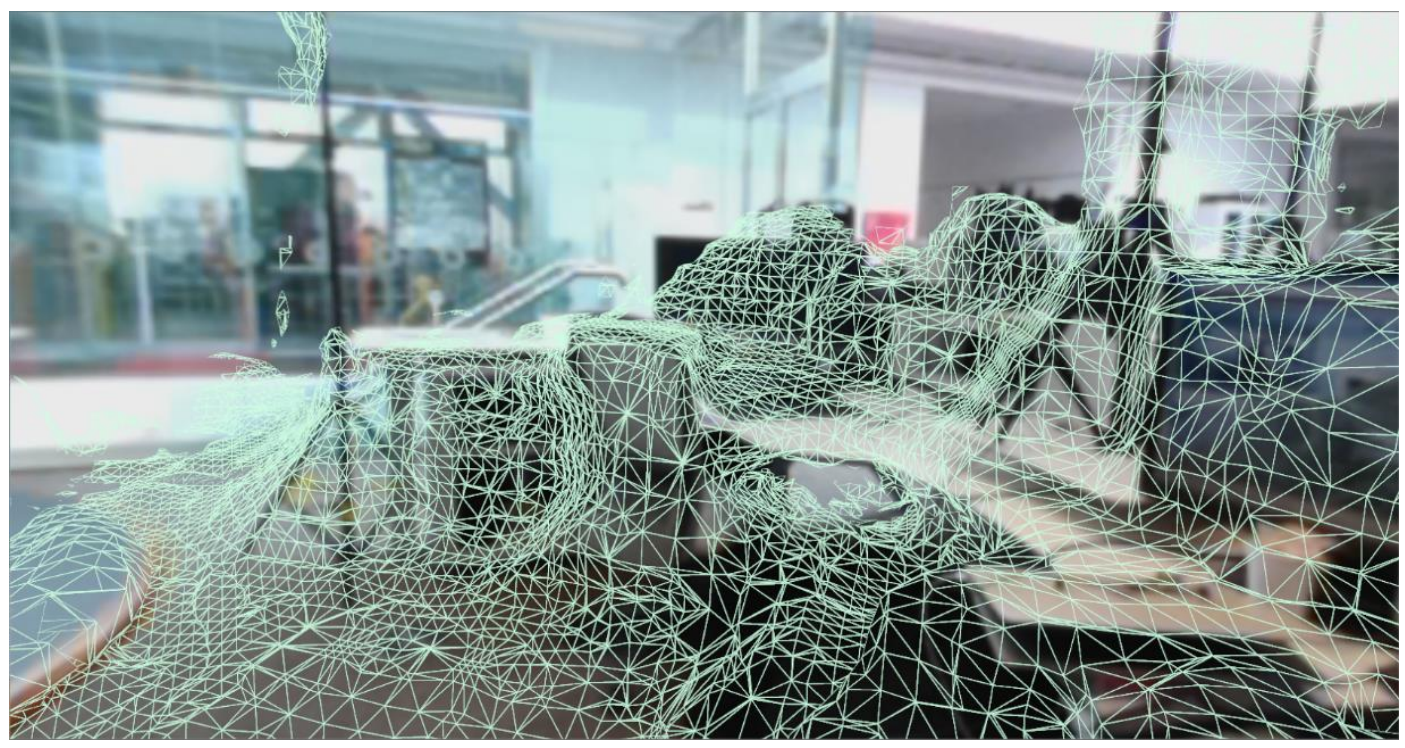

Figure 18 Spatial scanning result

After generating the desired 3D meshes, select the "Save" button to store the 3D models, and then reload models by clicking "Enable Mesh > Static (VR)" (Figure 19). As a result, the saved meshes will be loaded into the virtual space, as shown in Figure 20.

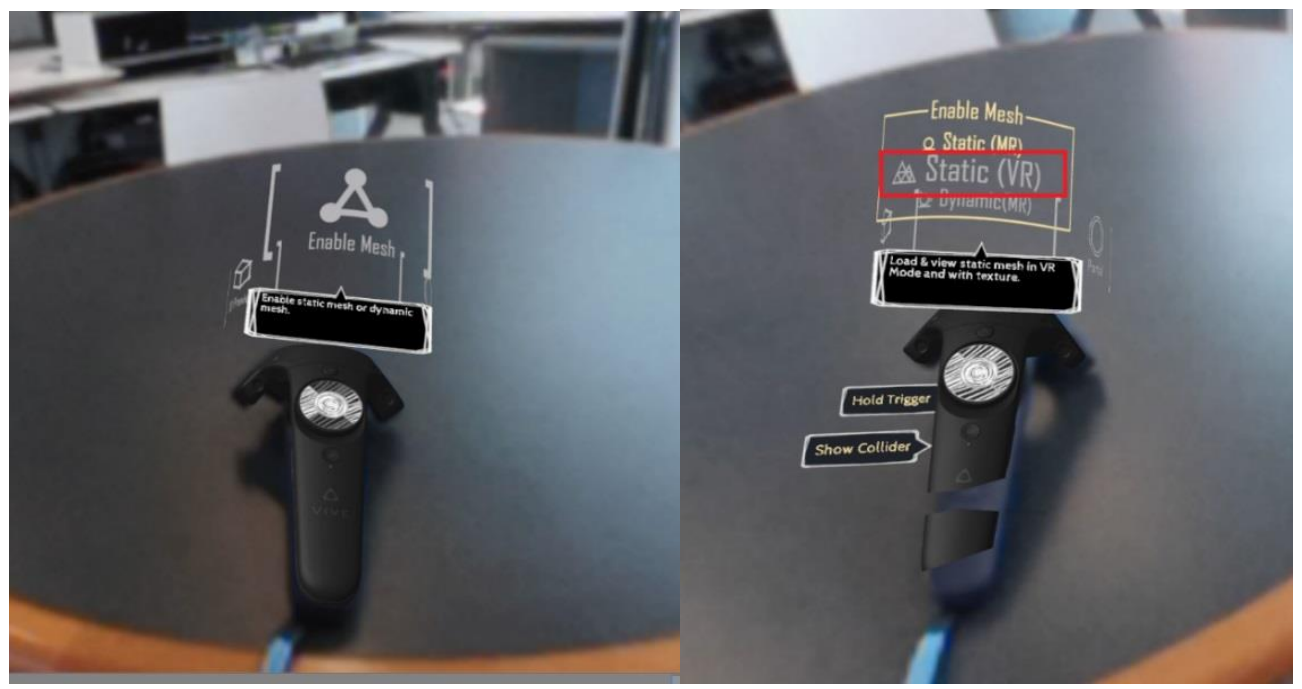

Figure 19 a) Enable Mesh

b) Static (VR) 


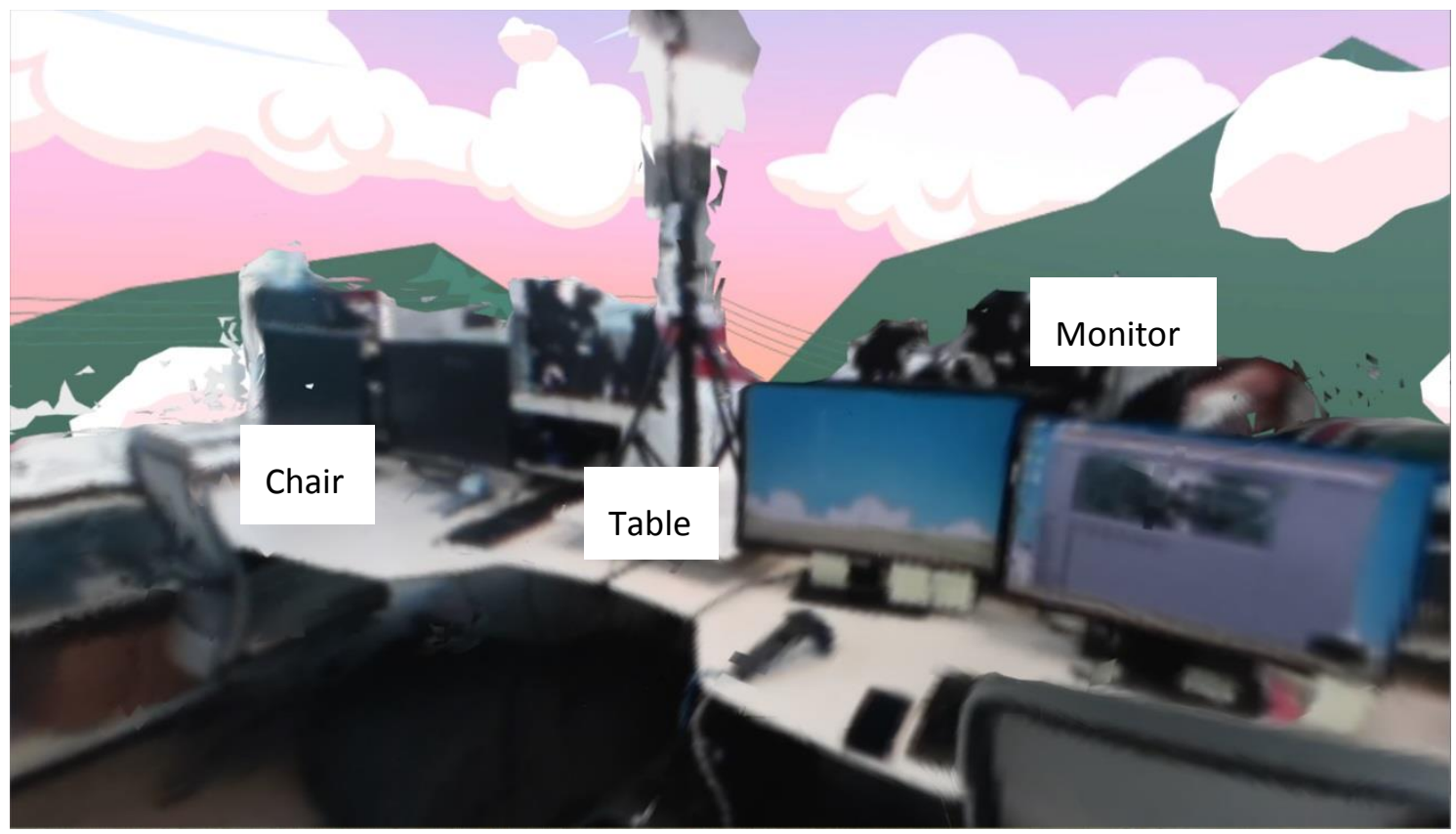

Figure 20 Loaded meshes

The created meshes contain the geometrical information of the real physical objects, and they are decorated with low-resolution pictures taken by the front camera. However, the virtual objects laid behind the meshes are blocked, which can significantly reduce the immersive experience in VR. Therefore, the produced meshes in this step were still entirely different from the desired final prototypes. To achieve the grid-like meshes as the Figure 21, new textures needed to be designed and applied to the 3D meshes.

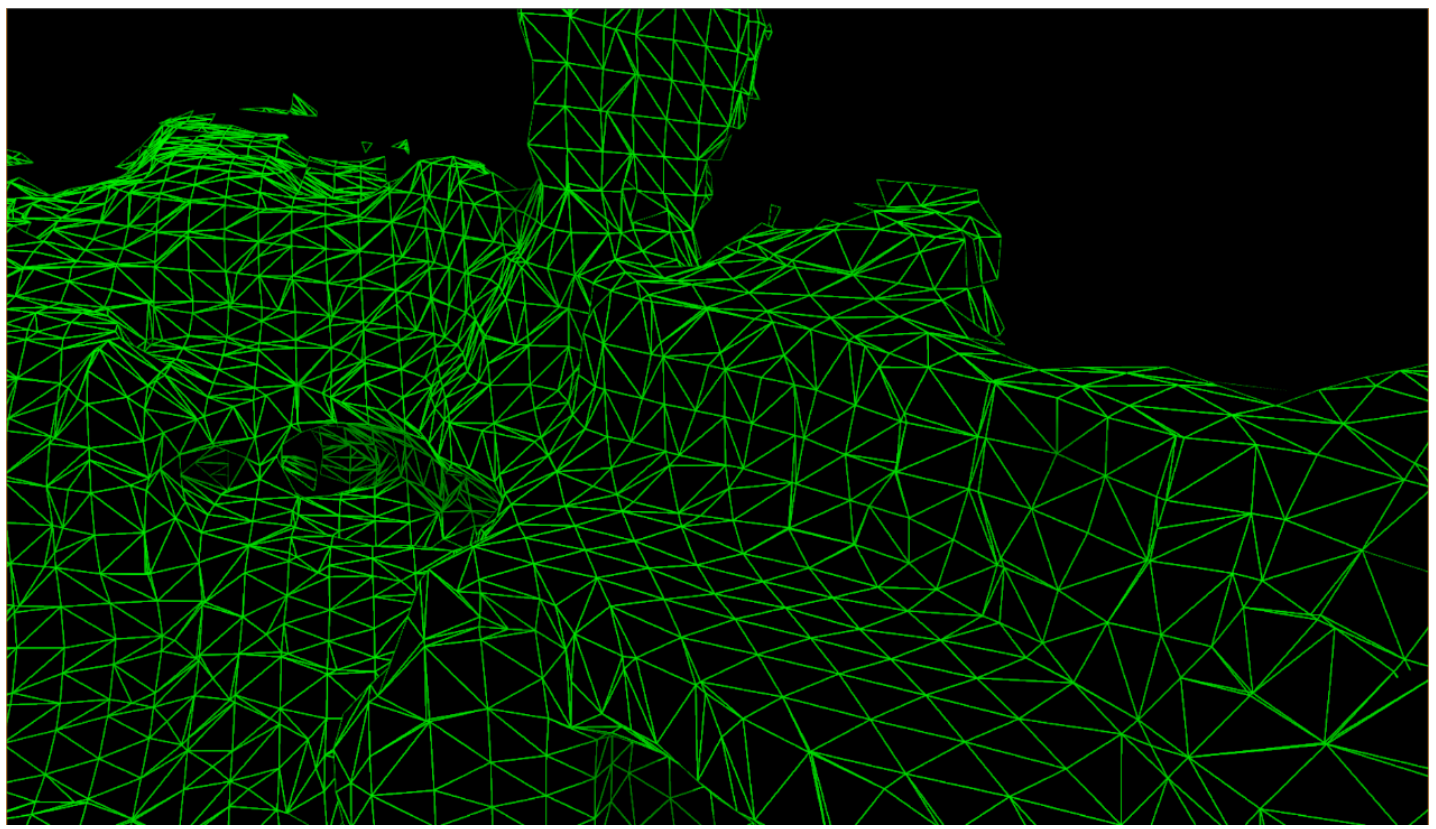

Figure 21 Target style of 3D grids

\subsubsection{Grid-like Material Generation}

There is a typical workflow to follow to render a new texture material in Unity, as seen in Figure 22. A collection of 3D coordinates named vertices construct 3D models, and each model must include a material which contains 
a shader. To detailly customize the look of 3D models, a suitable method is to edit values for properties in a shader.

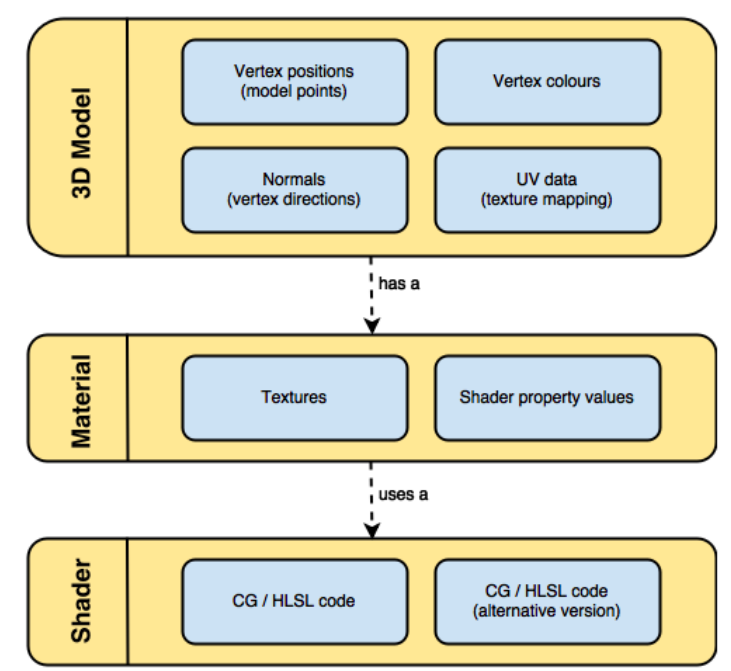

Figure 22 Rendering workflow of Unity ${ }^{15}$

By further exploring the ViveSR SDK, we found that a material named "WireframeMaterial" is attached to the mesh model, and a Shader called "WireframeGeoShader" is employed by the material to define how to render each piece of the generated meshes. To achieve a grid-like style, the corresponding properties in the shader should be constructed first. In our script, line strip primitives to a stream-output object were appended. In this way, the original geometrical information of meshes produced in the scanning step could be preserved, including the shape and depth information, but the meshes were rendered with line strip style instead of lowresolution pictures. Consequently, the virtual objects hidden behind the meshes would not be obscured any more. Principally, the result shown in Figure 23 was the embryo model desired for this project.

\subsubsection{3D Virtual Monochrome Grid (3DVMG)}

By appending the customized 3D wireframe material with a solid colour (cyan) to the 3D grid, the first interface 3D Virtual Monochrome Grid was generated, and then it was augmented into the VEs. In this case, the wireframe retained the geometrical data of the real environment, while players could have a glimpse of virtual content through the triangle holes.

${ }^{15}$ https://unity3d.com/learn/tutorials/topics/graphics/gentle-introduction-shaders 


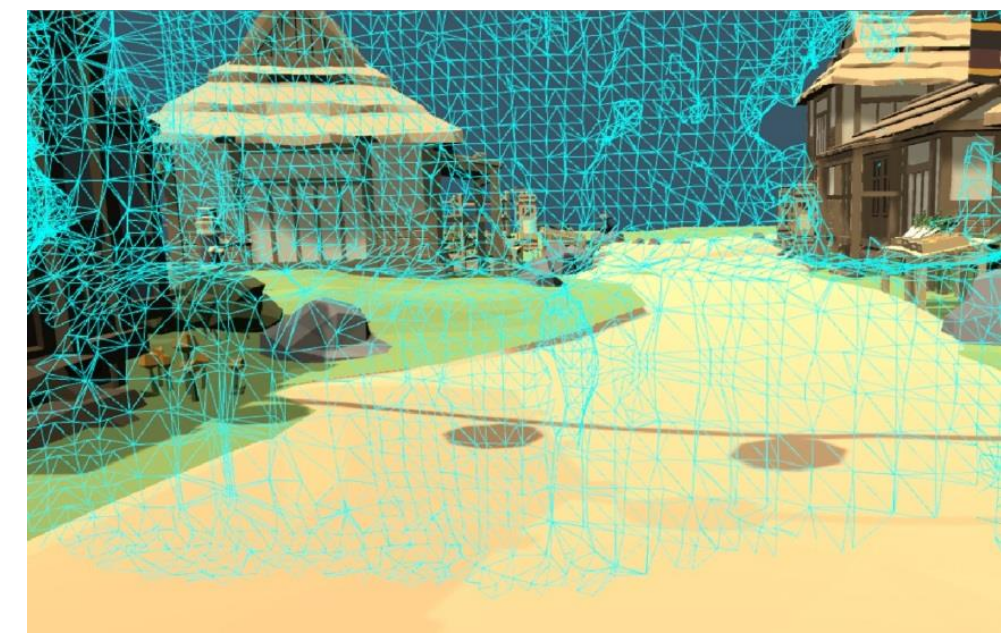

Figure 23 Result of appending the new shader with one solid colour

\subsubsection{3D Virtual Coloured Grid (3DVCG)}

Since the second aspect that we wanted to investigate in this project was the performance of colour visualization in improving the navigation safety, a shader that could tint meshes with different colours according to the depth values was investigated. Based on the first grid, a second shader named "ColouredWireframeGeoShader" was developed. It is capable of applying different colours to various pieces of grids by calculating the distance between the stereo front camera and meshes. To be specific, colours are switched for various distance ranges controlled by the above distance values through assigning different colour values in the script.

Initially, seven colours extracted from the spectrum (red, orange, yellow, green, blue, indigo, violet) were deployed (Figure 24). However, feedbacks collected from the pilot study showed that multicolour caused confusion, uncomfortable feelings and distraction.

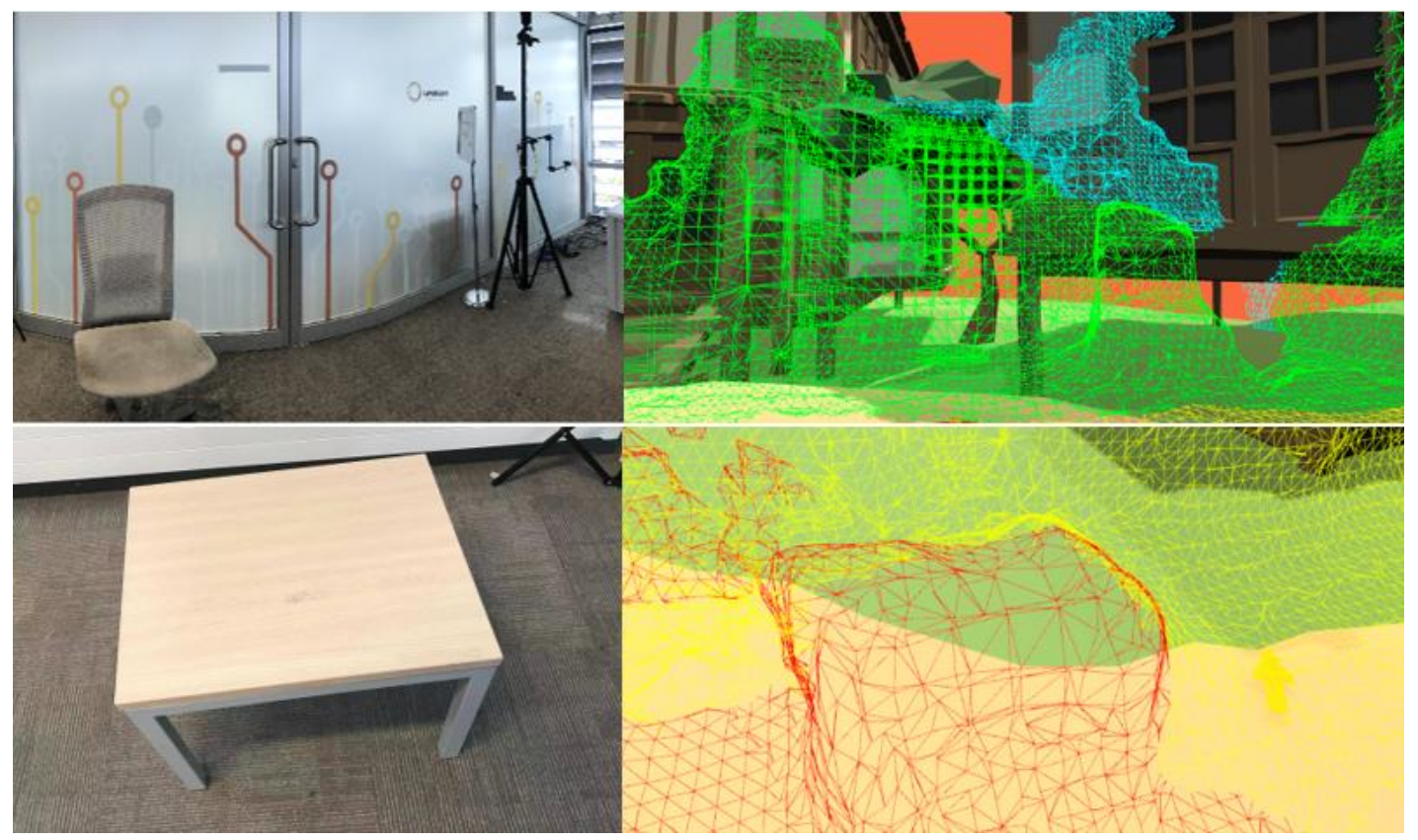

Figure 24 3D Coloured Grids based on different distance

Therefore, the applied colours were reduced to three, such as red, green and blue. They were used to present near, middle and far distances respectively. For example, the red part on the screen means the user is very close to the object, and extra cautions are required during the interaction, while the green part indicates middle 
distances and objects tinted with blue mean that they are far away. The final appearance of the 3DVCG is shown as Figure 25, and with its help users can understand the spatial layout of the surrounding objects and then avoid any potential stumble with less confusion.

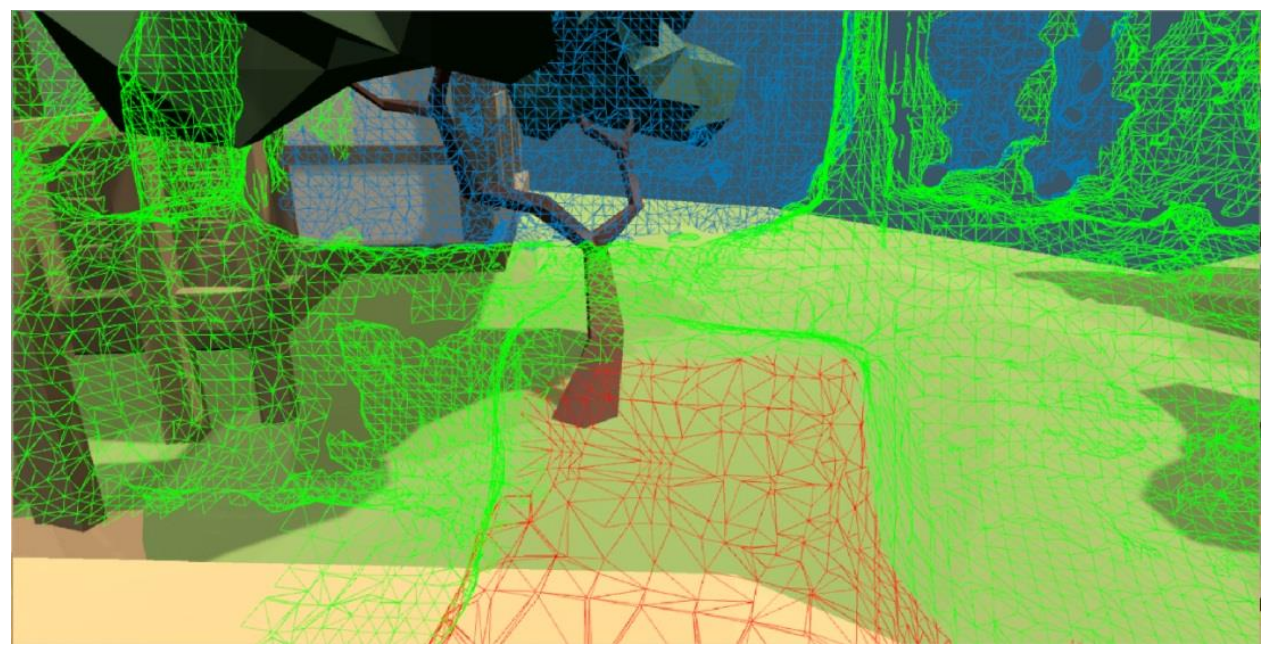

Figure 25 3D Coloured Grids

\subsubsection{Other VR Factors}

\subsubsection{Collision Detection}

After finalizing the design of Virtual Safety Interfaces (VSIs), the following plan was to visualize grids when users collide with them in the AV space. In this study, the VSIs were intended to be visualized regardless of the location of obstacles, as long as the distance between users and obstacles are out of safety ranges. In contrast, the exhibiting rules of our interfaces are different from the one employed by the Chaperone, and we could not simply set VSIs to active status in Unity through calculating the distance from the player to the boundaries. Instead, we utilize the OnTriggerEnter () and OnTriggerExit() functions in Unity. If the player collides with or leave the grids, VSIs will appear or disappear automatically. To achieve this, either of the two related game objects (grids and player) in Unity must have a collider and Rigidbody components. Another determinant element to call these functions is setting one of the game objects as a trigger. Here the capsule collider attached to the player was set to the trigger to activate the grids, since it could duplicate the movement of the player better than other types of collider. When the player is about to crash into the real objects, the corresponding capsule collider will collide with virtual meshes, and then the grids will be displayed, as shown in Figure 26 . The grids will disappear after the player leave the collision area, as shown in Figure 27. 


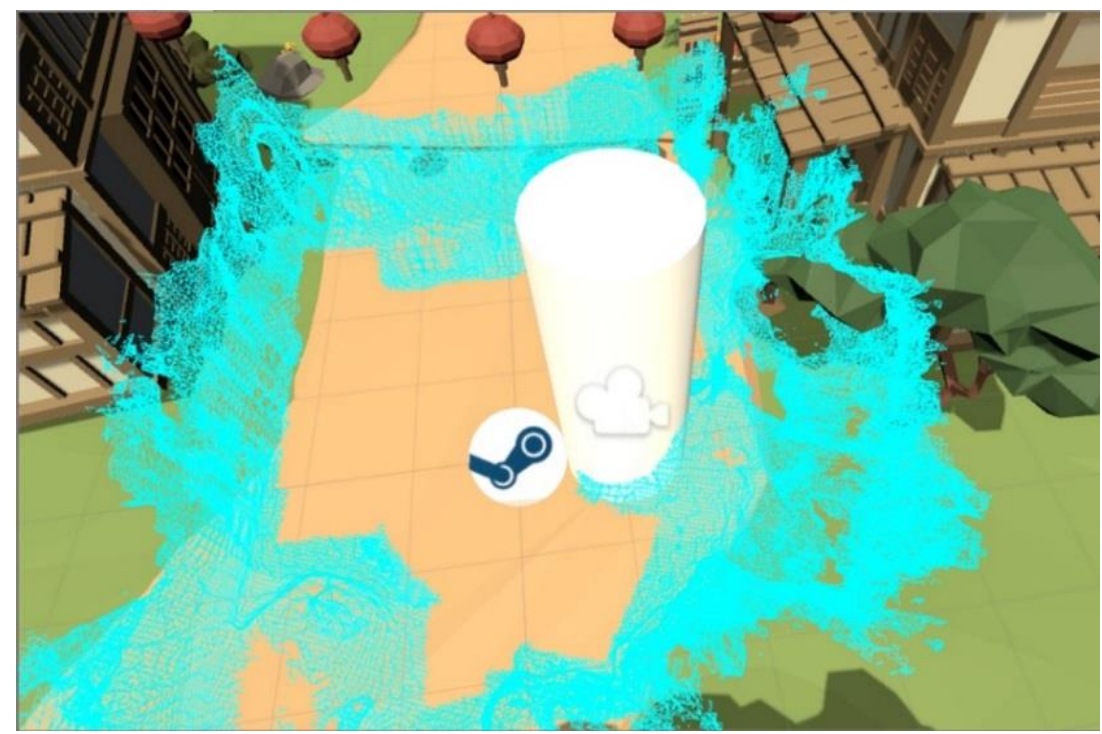

Figure 26 Collision detection - Enter

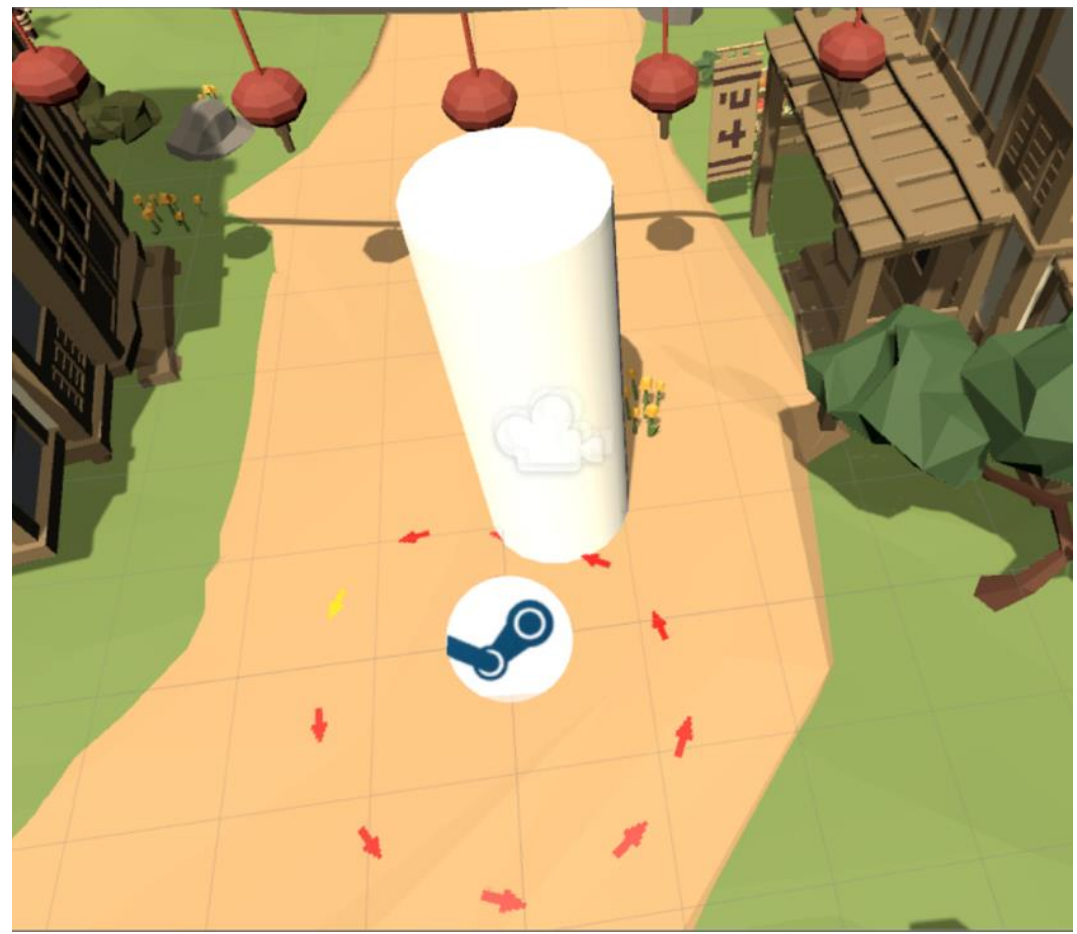

Figure 27 Collision detection - Exit

Apart from that, for offering better user experience and ensuring the smooth running of the demo, the design of the players' collider was confirmed after thoughtful considerations. For example, to ensure the capsule collider can behave as players do, the rotation around the $x, y$ and $z$-axis were all set to 0 degrees. Hence, even though players rotate or bend their heads, the corresponding collider can still keep vertical. Otherwise, the rotation of the cylinder will cause incorrect collisions, which results in undesirably demonstrating VSIs. Another detail to mention is the height of the cylinder was set to two meters to suit the heights of different subjects. This is to ensure that the bottom of the cylinder will not be too high to touch the mesh collider for people who are taller on average. Then the VSIs will not appear or show up later than the expected time. The last detail taken into consideration was about the radius of the collider. After trying the HTC Tron interface, it was found that the interface comes up at $50 \mathrm{~cm}$ from the edge. Thus, the radius value was set to this threshold. Therefore, 
three warning interfaces can provide the same reaction distances for users and the fairness in this aspect is guaranteed.

\subsubsection{Fade Effect}

After testing the first version of the interfaces, we found that the sudden appearance of the safety grids while the user was navigating in the virtual world may cause an unpleasant shocking feeling, which was affirmed by the results of previous research [27]. In addition, the existing Chaperone and Tron interfaces both can gradually appear or disappear when players enter or exit the boundaries. To ensure that our interface can provide the same smooth user experience and reduce users' level of discomfort, a fade effect was added to the interfaces. When the player enters or exits the meshes, the opacity value of the corresponding interface will be gradually increased or decreased respectively. To implement this effect, the original "SetActive" method was discarded, and an alternative "FadeOpacity ()" function was used to increase or decrease the current opacity of the used material. Values of the opacity were then passed to the "_Transparency" property in the shader via using a "Material.SetFloat()" function. As a result, the transparency of interfaces could be changed according to the player's movement. From our pilot study, users reported that the brightness of VSIs was too harsh and led to feelings of discomfort if staring at it for a long time, so we adjusted the range of the opacity being from 0.0 to 0.8 .

\subsubsection{3D Models Merging}

After the completion of above steps, a final test was conducted. The result showed that the collision detection was not flawlessly executed but with lots of extra collision detections. The reason was the default static meshes generated by HTC Vive Pro consisted of multiple pieces instead of a single one, and these meshes overlaid with each other. When the player collides with the meshes, the Enter and Exit functions will be called more than one time since several collisions between the player and the different parts of grids are detected. As a result, the meshes keep disappearing and appearing frequently. For better performance, we imported these meshes into the "blender" and merged them into one single 3D mesh (Figure 28). After that, there will be only one detected collision every time when the player walks into the meshes, and the visualization of interfaces will be more reliable.

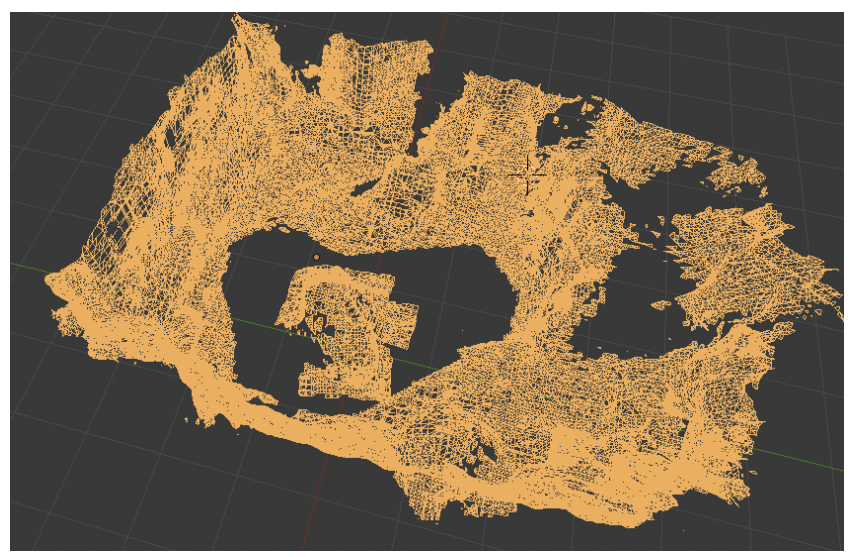

Figure 28 Merge pieces into one mesh

In conclusion, we used the HTC Vive Pro headset as our demonstration VR device, which supports 6 DoF motion tracking and stereo camera-based depth sensing for spatial environment mesh reconstruction. Our two 3D VSIs were developed based on these features. Specifically, the HTC Vive Pro is able to scan objects in the real world and save them as static meshes, we then improved these 3D meshes by applying the grid texture and assigning different colours to meshes to create two safety interfaces, 3D monochrome grids and 3D coloured grids. 
Meanwhile, instead of displaying the warning meshes when players approach the edge of a playing area, our grids will become visible as long as collisions between the player object and virtual meshes happen. A cylinder collider used to represent the user is attached to the headset. When the cylinder collides with the grids, the safety interfaces will become visible to the player to remind them of approaching obstacles around. When it exits, the safety interfaces will become totally transparent. 


\section{Chapter 4 User Evaluation}

This chapter describes an official user study using the created prototype to investigate two research questions in three sections: the evaluation purpose, the hypotheses and the user experiment design.

\subsection{Evaluation Purpose}

There are two purposes of this user study, 1) we wanted to investigate whether the safety of VR navigation can be improved with depth sensing interfaces, 2) we were particularly interested in finding out whether adding colour clues based on depth values to the depth sensing interfaces can improve interaction safety more than the monochrome version.

\subsection{Hypotheses}

Three safety interfaces are explored in our experiment, 1) 2D outlines of surroundings, 2) 3D Virtual Monochrome Grid, and 3) 3D Coloured Grids, and we have two null hypotheses for the experiment:

- Hypothesis 1 (H1): There is no significant difference for users to perceive obstacles in the real world between with and without depth information interfaces.

- Hypothesis $2(\mathrm{H} 2)$ : There is no significant difference for users to perceive obstacles in the real world between with or without colours visualization interfaces.

\subsection{User Experiment Design}

\subsubsection{Within-group Design}

There were two studies in our experiment. A within-group design was adopted in our two experiments for this study. The learning effect was very limited. Even though positions of obstacles in the real space were not changed during each study, the possibility that participants can remember the exact locations of each object based on the last testing trail would be very low. Participants may remember the vague positions of obstructions according to the previous environment meshes, which they have learned in the practice trail before the official test.

To evaluate the $\mathrm{H} 1$, a 3 by 1 comparison experiment among three collision avoidance methods was conducted, which studied users' ability to perceive obstacles in real space, as shown in Table 1.

Table 1 Conditions for Experiment 1

\begin{tabular}{|l|l|l|l|}
\hline & Condition A & Condition B & Condition C \\
\hline $\begin{array}{l}\text { Users' ability to perceive } \\
\text { obstacles }\end{array}$ & 2D outlines of surroundings & $\begin{array}{l}\text { 3D Virtual Monochrome } \\
\text { Grid }\end{array}$ & 3D Virtual Coloured Grid \\
\hline
\end{tabular}

To evaluate $\mathrm{H} 2$, a 2 by 1 comparison experiment between two collision avoidance methods was carried out, which had the same target as study 1 in a situation that subjects' distances to the obstacles are shorter than average, as shown in Table 2.

Table 2 Conditions for Experiment 2

\begin{tabular}{|l|l|l|}
\hline & Condition A & Condition B \\
\hline $\begin{array}{l}\text { Users' ability to perceive } \\
\text { obstacles }\end{array}$ & $\begin{array}{l}\text { 3D Virtual Monochrome } \\
\text { Grid }\end{array}$ & 3D Virtual Coloured Grid \\
\hline
\end{tabular}




\subsubsection{Latin Square}

Since both experiments were within-subject designs, to eliminate the potential order effect, we used Latin square design to counter-balance it. For study 1, there were three conditions. Thus a 3 by 3 Latin square design was used (as shown in Table 3).

Table 33 by 3 Latin Square

Where $A=2 D$ outlines of surroundings; $B=3 D$ Monochrome Grids; $C=3 D$ Coloured Grids

\begin{tabular}{|l|l|l|}
\hline A & B & C \\
\hline C & A & B \\
\hline B & C & A \\
\hline
\end{tabular}

Similarly, for study 2 , a 2 by 2 balance Latin square design was used (as shown in Table 4).

Table 42 by 2 Balance Latin Square

Where $A=3 D$ Monochrome Grids; $B=3 D$ Monochrome Grids

\begin{tabular}{|l|l|}
\hline$A$ & B \\
\hline$B$ & A \\
\hline
\end{tabular}

\subsubsection{Experimental Setup}

a) Physical environment setup

The study was conducted in the Student Room of the HIT Lab NZ, and the environmental arrangement of the two studies are shown as in Figure 29 and Figure 30.

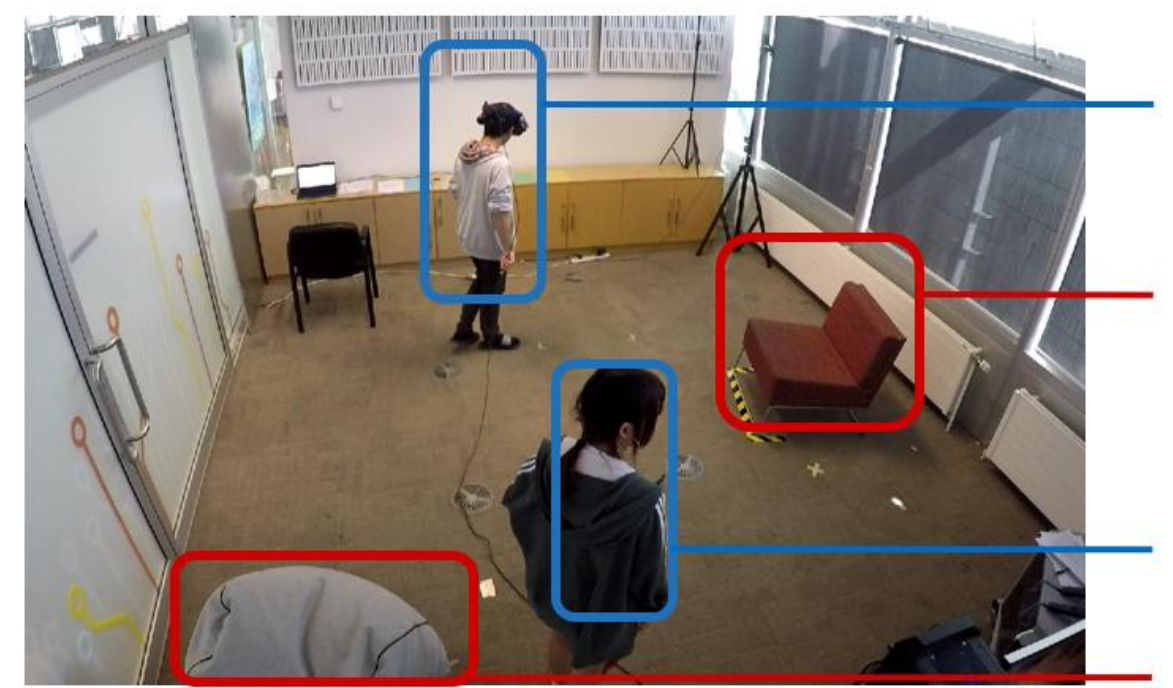

Figure 29 Experiment 1 Setup
Participant wearing HTC Vive Pro headset

Ubstacles - Chair

Guidance

Obstacles -Beanbag 


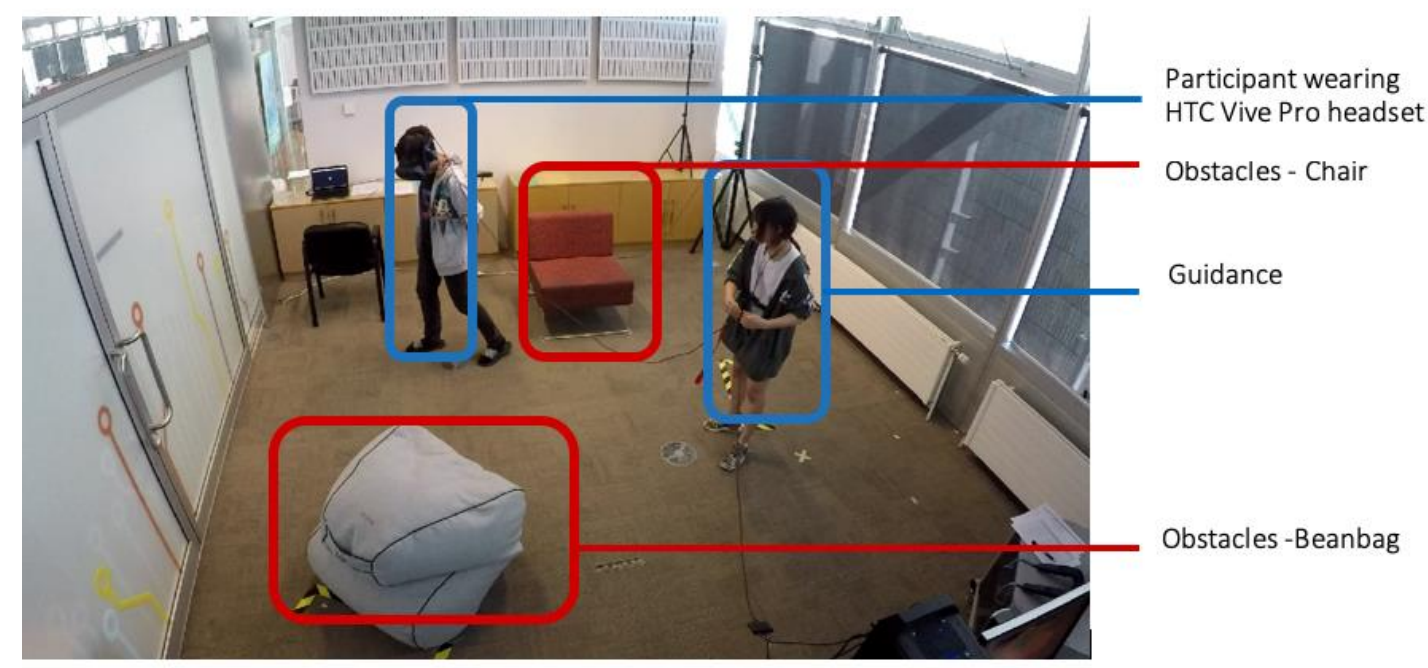

Figure 30 Experiment 2 Setup

The main difference of these two environment setups is the positions of obstacles. In Study 1, both obstacles were placed outside the boundaries of the playing areas to ensure that when subjects approached real objects, the safety interfaces appeared to give them a warning message in each condition. In Study 2, since we wanted to investigate people's performance in when the distances between obstacles and themselves are very short, those real objects were positioned inside the playing areas.

During the experiment, subjects wore the HTC Vive Pro headset mentioned before, and navigated the virtual world by following predetermined paths in VE. A guide was always at a distance of intervention to prevent participants from accidentally bumping into physical objects. If at any time during the experiment the experimenter detected any obstacles that may endanger the participant, the participant was notified, and the experiment was paused until the danger was removed. Starting points of the participants in the two studies were marked on the floor, and there was a display screen reflecting the content that subjects saw in the headset.

\section{b) Virtual environment}

Figure 31 demonstrates the virtual space that subjects were exposed to. In this virtual space, they found that they were standing in a virtual garden with arrows marked on the road. The actual scale of the virtual path shown in Figure 31 was 3.0 metre by 3.0 metre in the real environment. The yellow arrow indicates the starting and ending points.

Participants were asked to follow a specified path indicated by arrows to explore the VEs in each condition. Multiple paths were generated to avoid learning effects. At the left side of Figure 31, five paths can be found, three for experiment 1 and two for experiment 2. Directions of these paths were randomized without replacement. The displaying order of these paths also followed the 3 by 3 and 2 by 2 Latin Squares mentioned before to counter-balance the order effect. 


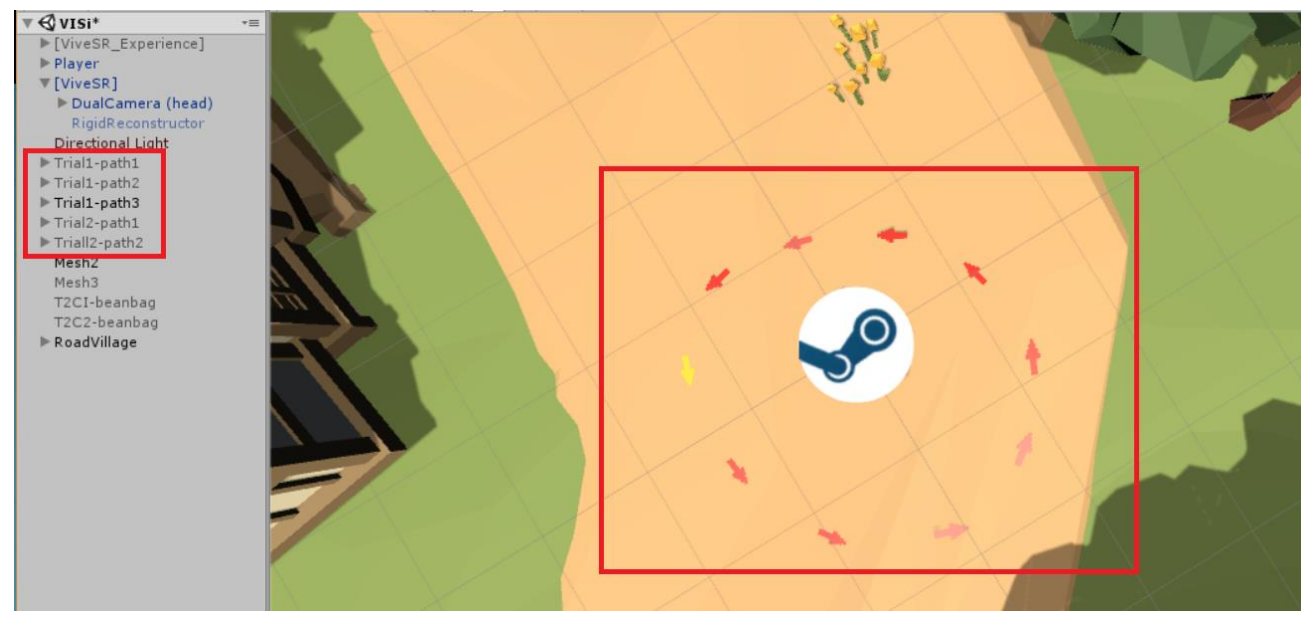

Figure 31 Virtual Environment Setup

\subsubsection{Procedure}

Overall, the whole experiment lasted about 40 minutes, and two studies of it followed the procedure described below.

1. Firstly, after the participant was welcomed, a general overview and explanation of the project were given. Then, participants were asked to read the information sheet and sign the consent form. These two documents are provided in Appendix A.

2. They were asked to watch a training video with the explanation of three safety interfaces, with which they would be exposed to during the experiment later.

3. Then, a pre-experiment questionnaire that aims at collecting their demographic information and their personal experience in playing VR was presented to answer. The document is provided in Appendix B.

4. Participants had one or two minutes to have a look at the testing environment, and they were made aware of obstacles, before they were led to the start point marked on the floor.

5. After running the prototype, participants put on the headset, and the researcher gave a brief introduction about the task. Subjects were offered opportunities to practice walking in the virtual space.

6. At this point, subjects were ready to start the formal experiments. In the first study, they experienced three conditions in a sequence pre-defined by the Latin Square chart. Then participants answered a questionnaire for each condition to rate their experience after they completed each task.

7. Before they continued to the next study, they needed to answer a post-experiment questionnaire to give feedback on their personal experience.

8. In the second study, participants experienced two conditions in an order defined by the Balance Latin Square chart, and repeated the procedure they did in Study 1, including answering a questionnaire for each condition and the post-experiment questionnaire.

9. In the end, there was a short informal interview to discuss their navigating experience with different virtual safety interfaces.

\subsubsection{Experimental Task}

As mentioned before, there are two studies in this study, one having three conditions and the other containing two conditions. The tasks they were asked to do were different in two studies for answering our two hypotheses. Since the experiment is within-subject design, each subject went through five conditions in total.

\subsubsection{Study 1}

In Study 1, we intended to investigate whether the safety of VR navigation can be improved with depth sensing interfaces compared with the existing 2D method. It is well acknowledged that in a typical VR room-scale playing scenario, players have to empty the space at first, and the exiting 2D interface will only appear when users approach boundaries. In this study, since the 2D interface was used as a control, to ensure that when subjects 
approach real objects, the safety interfaces will appear to give them a warning message in each condition, both obstacles were placed outside the boundaries of the playing areas.

In this study, subjects were asked to navigate the virtual garden by following a virtual path, and to avoid real objects according to the warning given by safety interfaces.

Condition A: In Condition A, the participants stood on the start point (the yellow arrow), then followed the direction of arrows to navigate a virtual garden. Physical objects were placed in the way of the virtual path. Therefore, during their navigation process, they had the same opportunity to encounter obstacles regardless of the selection of virtual paths. In this situation, when they approached obstacles, 2D outlines interface was triggered and displayed (Figure 32). As a result, they could avoid obstacles in the real space according to the hints provided by this interface and continue their navigation task.

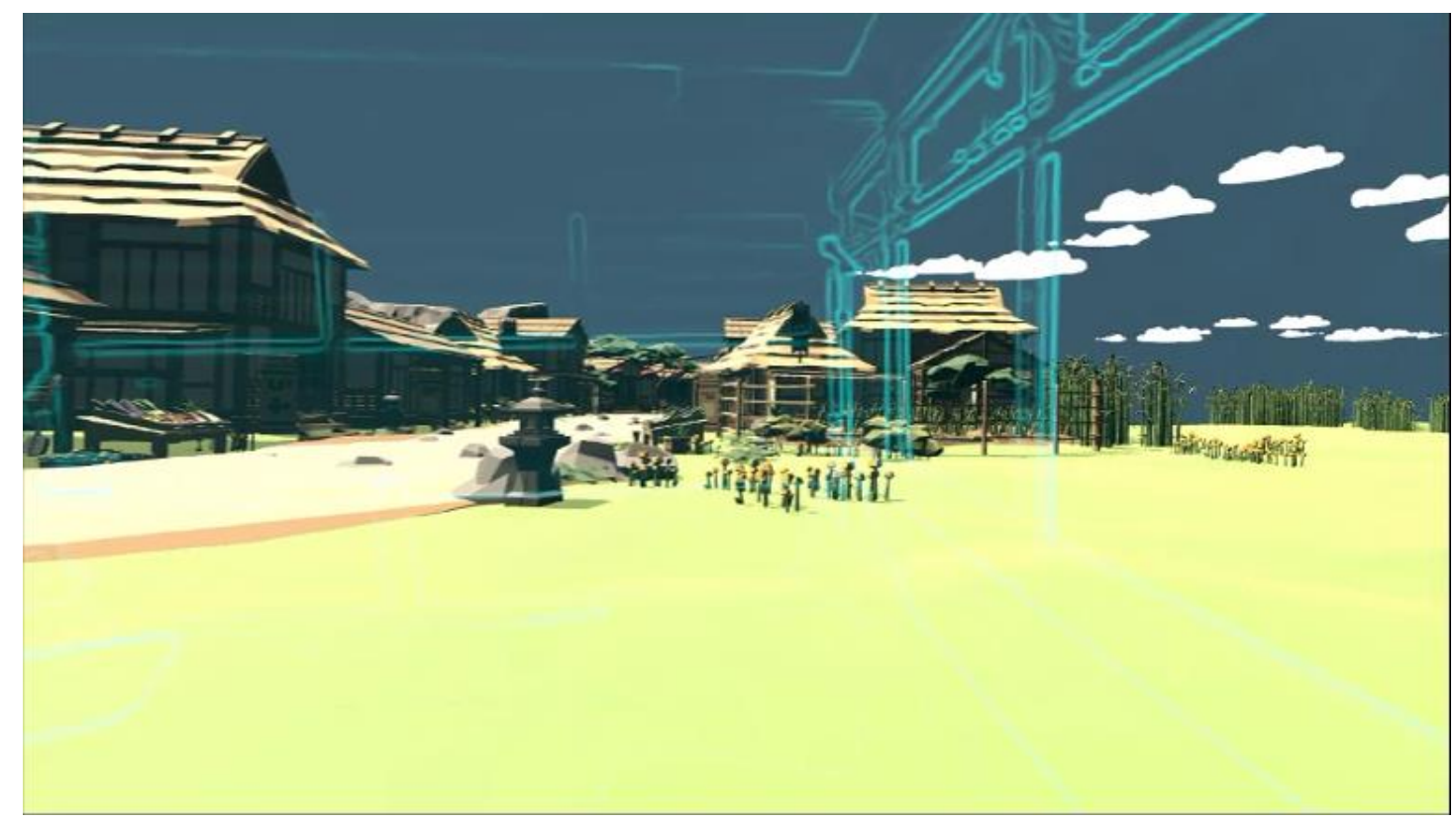

Figure 32 Study 1 Condition A - 2DOoS

Condition B: In Condition B, the participants were asked to complete the same task, but the path was slightly different, such as in the opposite direction. Another difference in this condition was that when they approached obstacles, they could see the 3D monochrome interface instead (Figure 33). They could walk away from obstacles in the real space according to precautions provided by this interface and continue their navigation task. 


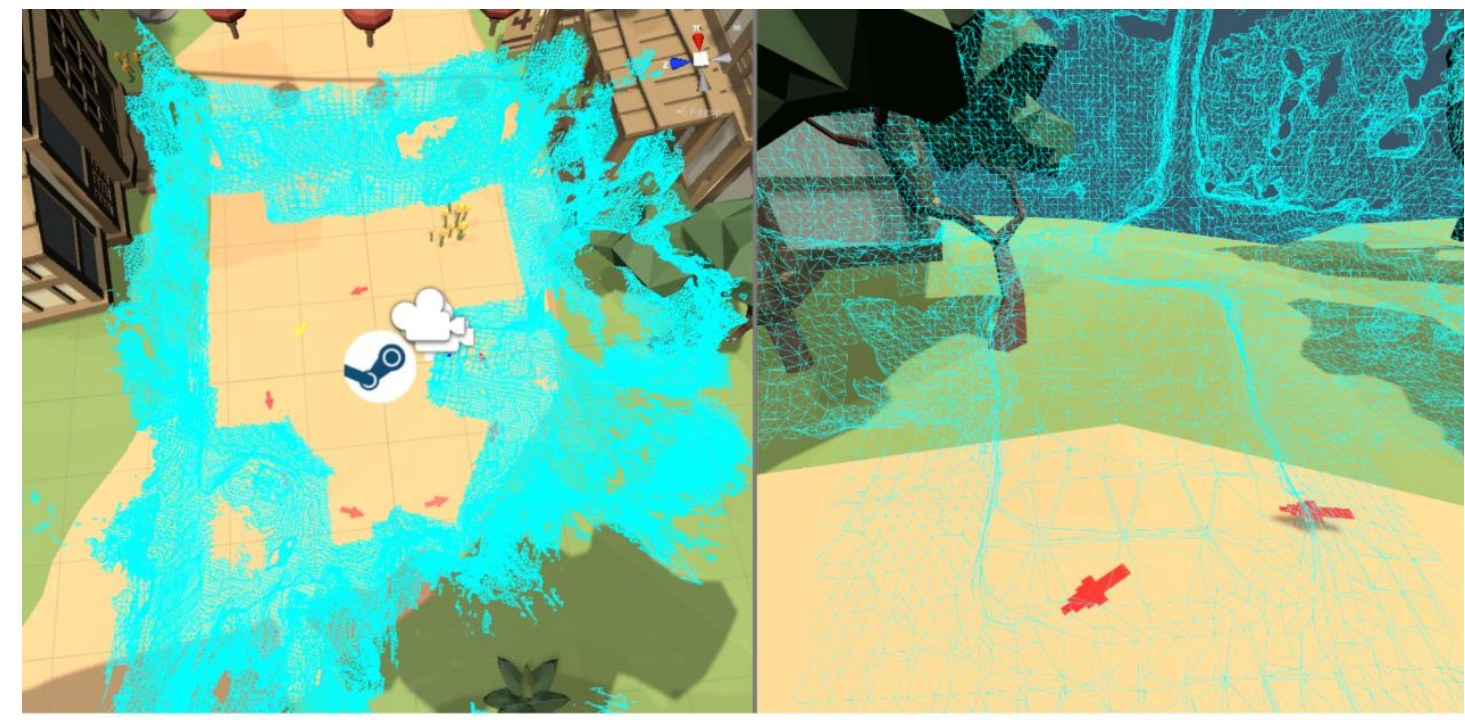

Figure 33 Study1 Condition B - 3DVMG

Condition C: In Condition C, the content of the task was unchanged, but the path and the virtual safety interface that the participants were exposed to were different as well. In this condition, they received the warning clues from the 3D coloured grids (Figure 34) and followed another different virtual path.

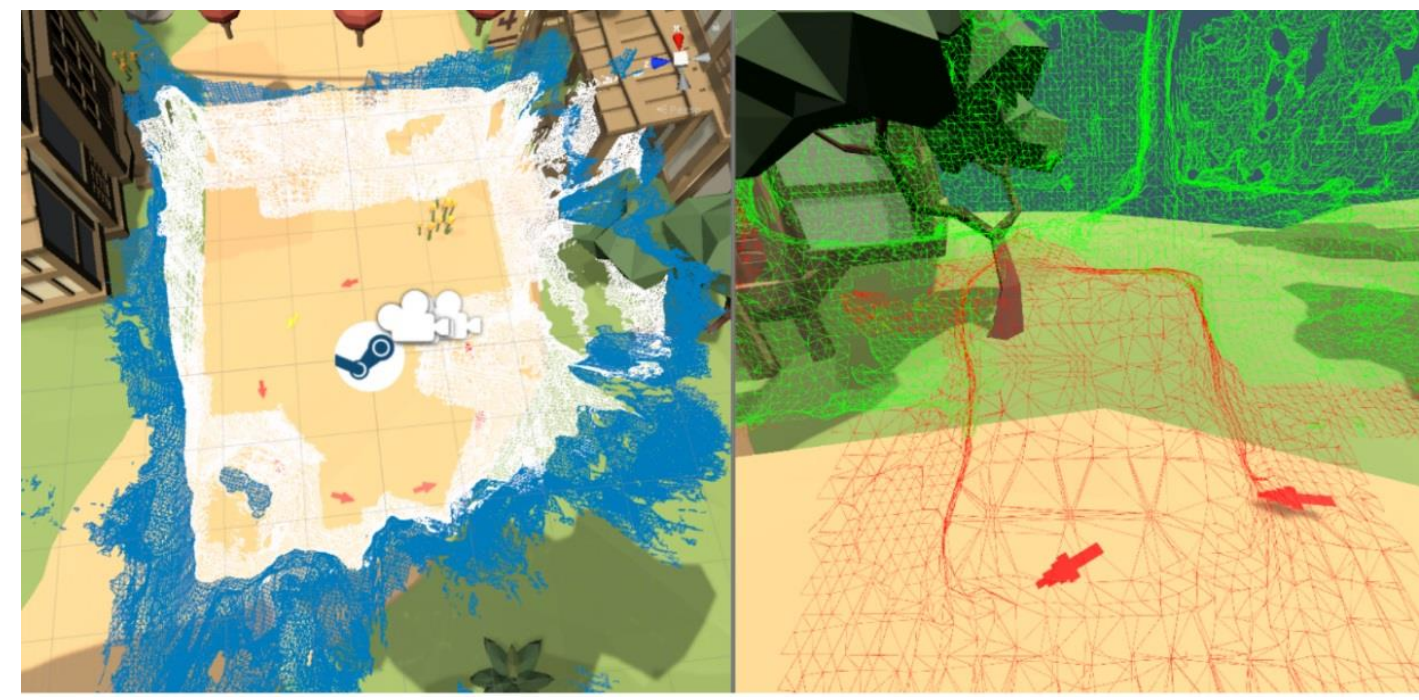

Figure 34 Study1 Condition C - 3DVMG

\subsubsection{Study 2}

In Study 2, the chair and beanbag were moved to different positions inside the playing area compared to their positions in Study 1. We wanted to investigate the usability of our proposed interfaces to see whether they can prevent subjects from colliding with obstacles, even though the real objects are positioned inside the playing area. We also intended to find out whether adding colour clues based on depth values to the depth sensing interfaces can improve interaction safety more than the monochrome version.

In this study, the task that subjects were asked to complete was different from that in Study 1 as well. Participants were asked to walk closer to obstacles, but to still try to avoid them. In reality, it is not uncommon that, users keep standing in the same position, even though the warning wall has already appeared. The reason behind this is that the guardian system appears in advance. Players can roughly predict the distances between them and obstacles via the interfaces. As long as they believe that they are still in a safe place, they will be reluctant to move. Therefore, we planned to explore whether the two 3D interfaces can assist users to perceive 
obstacles and whether the colour warning can have a better performance in helping users to step away from the furniture while the distances between are extremely short.

There were two conditions in Study 2.

Condition A: In Condition A, the participants stood on a new start point, and then followed arrows to navigate a virtual garden. The participant was asked to walk as close as they could to the obstacles in the way as long as they had confidence that they would not touch real objects. The possibility that they may collide with real obstacles still exited, due to the deliberately designed positions of obstacles. In this condition, if they approached the obstacles, the 3D monochrome interface would be visualized (Figure 35). Then, they could avoid obstacles in the real space according to the spatial information given from the interface.

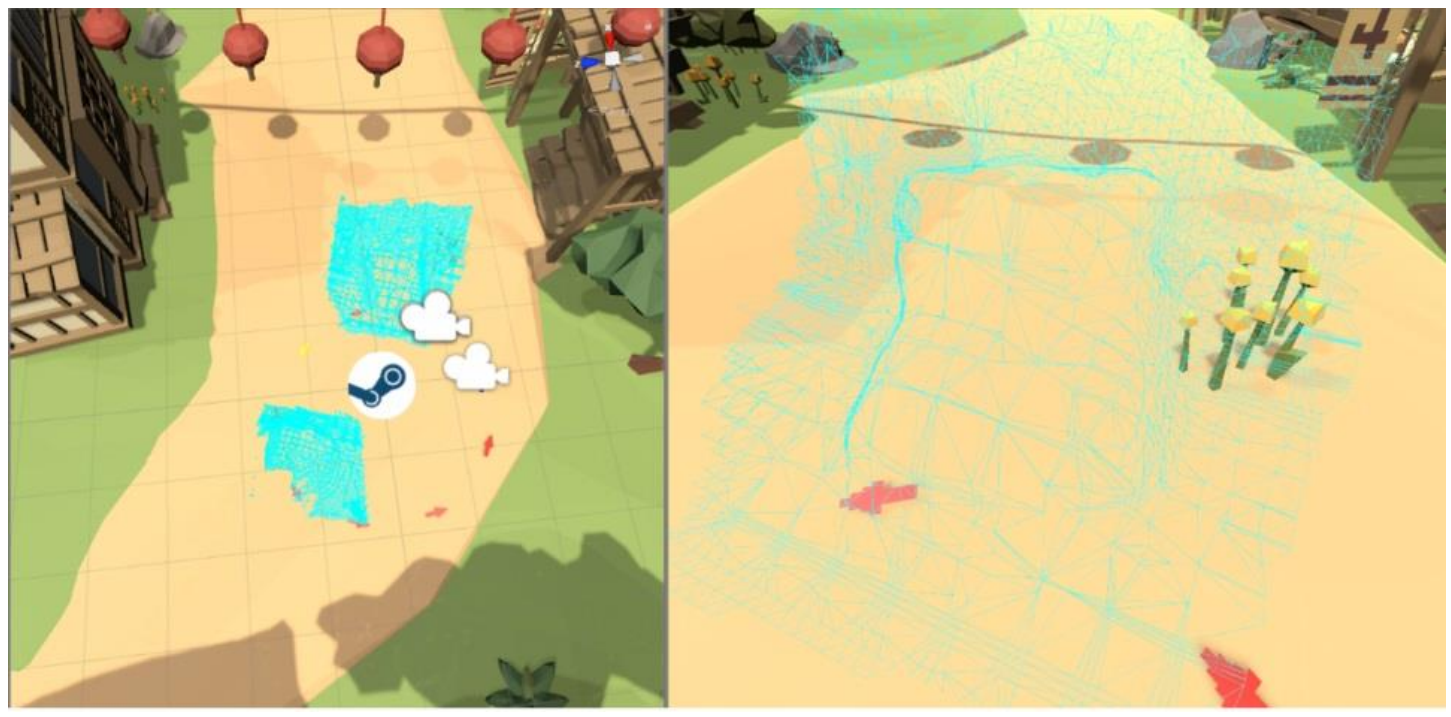

Figure 35 Study 2 Condition A - 3DVMG

Condition B: The participants were asked to complete the same task, but the path was slightly different, such as being in the opposite direction. Another difference in this condition was when they approached the obstacles, the interface triggered and displayed was the 3D colour one (Figure 36).

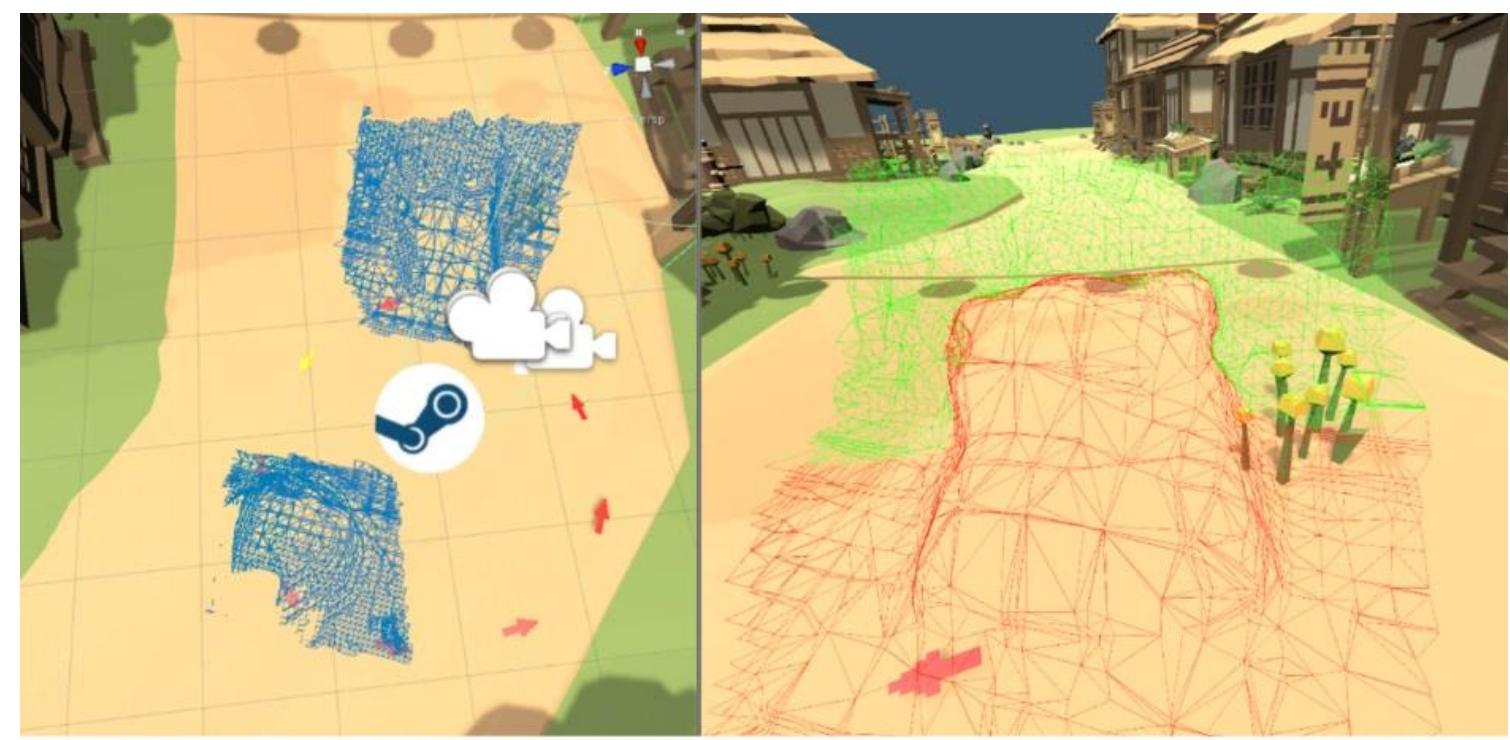

Figure 36 Study2 Condition B - 3DVCG 


\subsubsection{Measures}

There were five main measurements recorded for the experiment, sense of presence, understanding of realspace layout, collision count, closest distances to obstacles and users' preference.

It is well acknowledged that sense of presence is essential experience in VR. Thus, it is impractical to remove the immersion to achieve safety purpose. We were interested in studying whether our prototype would destroy or maintain the sense of presence in VR. One widely used method in previous research to measure sense of presence is igroup presence questionnaire (IPQ) [28], which measures four present components, such as general presence, spatial presence, realism and involvement ${ }^{16}$. There are 14 items in IPQ, and they are rated on a seven-point Likert scale [28]. These 14 items can be divided into three subscales and one independent general item, which are listed below:

- General Presence: assessing the general "sense of being there"

- Spatial Presence: assessing the sense of being physically present in the virtual environment

- Involvement: measuring the attention devoted to the virtual environment and the involvement experienced

- Experienced Realism: measuring the subjective experience of realism in the virtual environment

In this study, we used IPQ to measure sense of presence in each experimental condition, which is provided in Appendix B (From Q1 to Q14). Additionally, we were still interested in how much they understood of real-space layout, which was measured by a questionnaire used in Kanamori et al. work [29]. This questionnaire aims at evaluating users' sense of distances to obstacles and their confidence levels while walking in the VEs, which can be found in Appendix B (From Q15 to Q27). Apart from that, we focused on subjects' behaviours while they experience different safety interfaces. We observed whether they could successfully avoid obstacles through the spatial information provided by interfaces; If not, we recorded the times that they collided with real objects. The closest distances between subjects and obstacles were measured in real time, which were used to compare how subjects reacted to the warning information subjects received from the interfaces. In terms of the preference questionnaire, we wanted subjects to rank all tested interfaces according to their performance. Therefore, in this study, the collision counts, the closet distances to obstacles, subjects' preference, the IPQ, and the questionnaire of users' understanding of real-space information were measured in each condition, and they were adopted for the future analysis.

\subsection{Pilot Study}

We conducted a pilot study on three participants (males). All conditions and questionnaires were tested. It was a full-on study, in which users underwent the same procedure as the actual experiment. The pilot study was conducted to detect whether there was any unreasonable design of the interfaces or experimental procedure. The collected data was not included in the final result.

${ }^{16} \mathrm{http}: / /$ www.igroup.org/pq/ipq/index.php 
In the pilot study, we found the brightness of the two 3D interfaces was too intense, and the number of colours in the 3D coloured one was too high and had a disruptive effect. Therefore, we reduced the brightness and number of colours after consideration. 


\section{Chapter 5 Results}

This chapter presents the results from our user experiment. The data collected from each subject consists of a pre-experiment questionnaire, five per-condition questionnaires and two post-experiment questionnaires, collision counts and closest distances. The pre-experiment questionnaire collected participants' demographic information and their experience with VR, while the per-condition questionnaire gathered the quantitative data of each test, including sense of presence and understanding of the physical layout information. The postexperiment questionnaire collected preference rank and suggestions of all interfaces in each study. The collision counts recorded the total times subjects collided with real objects in each test, and the closest distances measured the closest distances between participants and surrounding objects during the navigating process in each test.

\subsection{Pre-experiment Questionnaire}

After obtaining approval from the human ethics committee, 24 university students were recruited for this user study. Among the 24 participants, 14 of them (58\%) were males, and 10 of them (42\%) were females. Participants' ages varied from 18 to 46 years old, with a mean of 29 and a standard deviation (SD) of 6.73. Out of 24 participants, four had no prior experience in VR, 13 had used VR headsets once, and seven were more frequent users. In the pre-experiment questionnaire, subjects' past experiences with VR were collected as well. Among the 24 subjects, 16 stated that they have bumped into obstacles during their VR experience, and eight had no such experience. For the widely used virtual safety interface (the grid wall), ten had not seen it before, and the last considered that they had seen it before. Before the actual experiment, they all had an opportunity to watch an introduction video of three virtual safety interfaces (VSIs) and had time to get familiar with the environment. Therefore, the results of this experiment are more likely to be generalizable.

\subsection{Results of Study 1}

\subsubsection{Quantitative Measures}

In Study 1, we had one independent variable (IV), the type of safety interfaces with three levels. The dependent variables were subjects' responses to IPQ, their understanding of the physical layout information questionnaire, their preference, the counts they collided with obstacles and the closest distance to obstacles. The results of these measurements are described below in details. In our study, an alpha level of 0.05 was used for determining the significant difference.

\subsubsection{Igroup Presence Questionnaire, IPQ}

The IPQ is a seven-point Likert scale to measure the sense of presence in the virtual space. In our experiment, 1 means strongly disagree, and 7 means strongly agree. There are four subscales in it, and all these components were analysed in our study. Since it is a Likert scale rating and the collected data is an ordinal type, a nonparametric test was used to interpret it.

We applied the Friedman-test $(\alpha=0.05)$ for the data analysis [29], and we also used the Bonferroni Method to adjust the $p$-value, which is 0.0167 . The results of four subscales in IPQ are interpreted separately in the following sections.

- General presence, GP 
From the data given by respondents, there is no significant difference existing among the three conditions $(p=$ 0.304 ) in terms of general presence. The mean values (shown in Table 5) prove all three interfaces were all scored highly, 2DOoS: 5.46 (SD = 1.062), 3DVMG: $5.75(S D=1.032)$ and 3DVCG: $5.75(S D=1.073)$ from scale 1 to 7 respectively, thus most subjects agreed that they had a sense of being there.

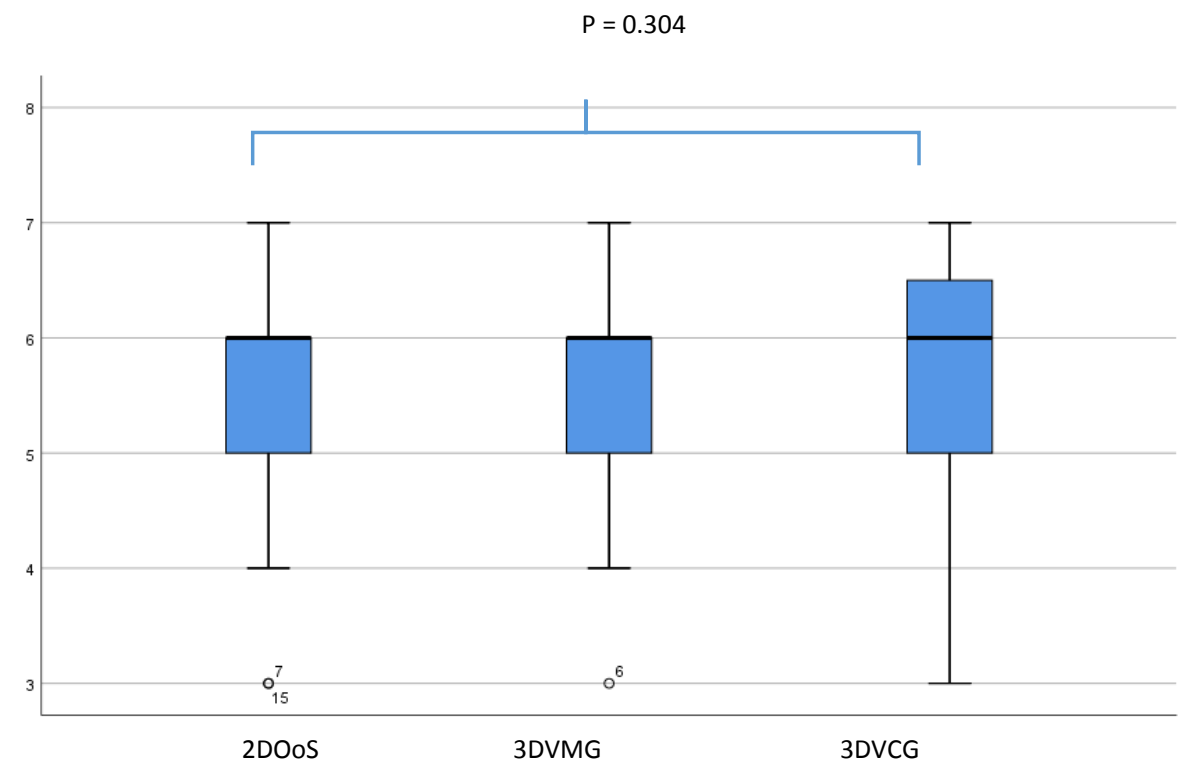

Figure 37 Results of GP in Study 1

Table 5 Mean values of GP in Study 1

\begin{tabular}{lll}
\hline & \multicolumn{2}{c}{ Mean (SD) } \\
\cline { 2 - 3 } Q1 Sense of being there & 2DOoS & $5.46(1.062)$ \\
\cline { 2 - 3 } & 3DVMG & $5.75(1.032)$ \\
\cline { 2 - 2 } & 3DVCG & $5.75(1.073)$ \\
\hline
\end{tabular}

- Spatial Presence, SP

Similarly, there is no significant difference found in 5 SP items (see Figure 38). In regard to sense of VE behind, sense of acting in VE and sense of being present in VE, their mean values demonstrated subjects agreed that the tested three interfaces offered spatial presence (all mean values > 5 for Q2, Q5 and Q6, see Table 6) and disagreed they were just watching pictures and not being in virtual space in all conditions (most mean values < 4 in Q3 and Q4). 


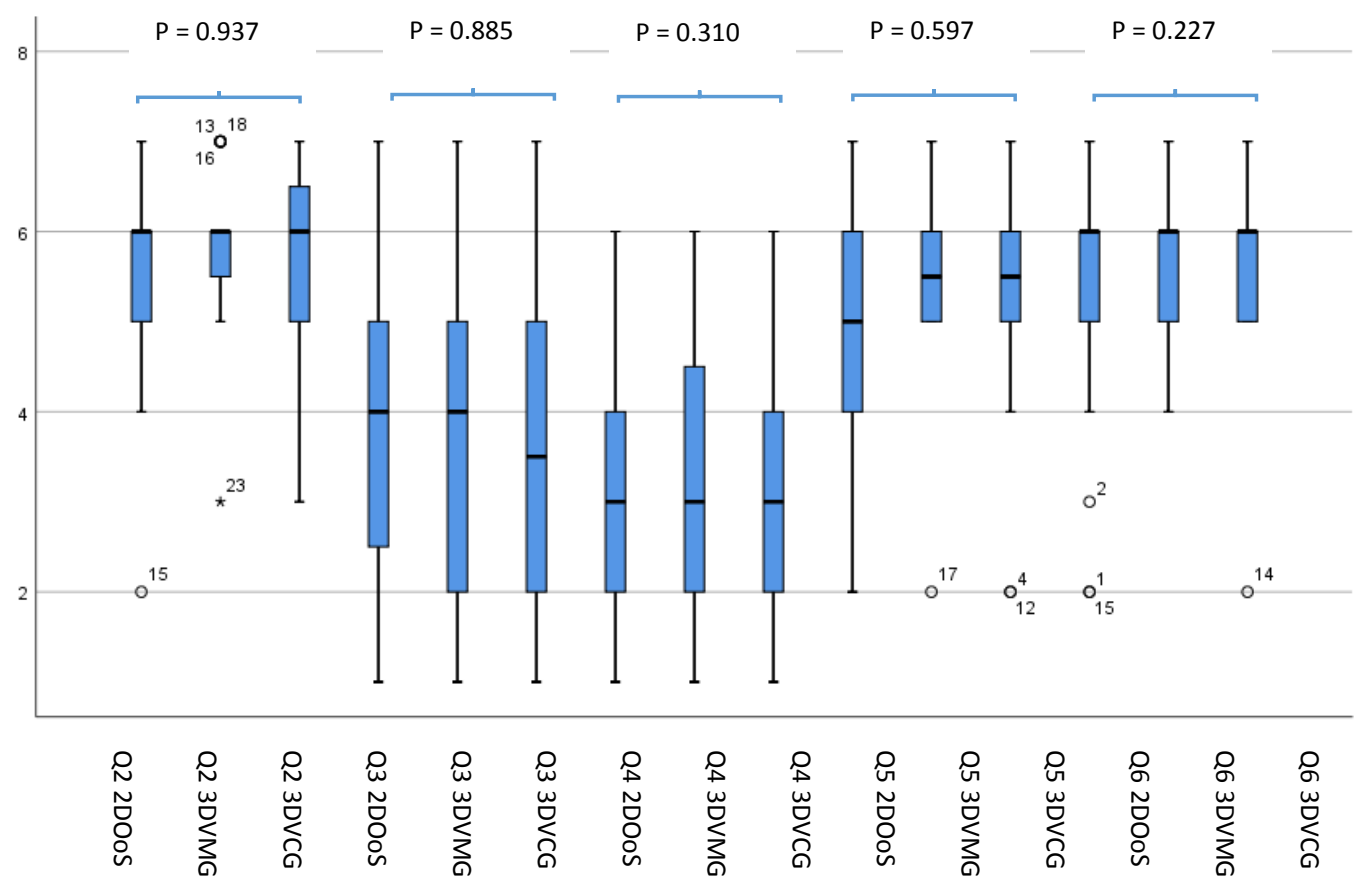

Figure 38 Results of Spatial Presence in Study 1

Table 6 Mean values of SP in Study 1

\begin{tabular}{|c|c|c|}
\hline \multirow{4}{*}{ Q2 Sense of VE behind } & & Mean (SD) \\
\hline & 2DOoS & 5.75 (1.113) \\
\hline & 3DVMG & $5.88(0.900)$ \\
\hline & 3DVCG & $5.75(1.032)$ \\
\hline \multirow{4}{*}{ Q3 Only pictures } & & Mean (SD) \\
\hline & 2DOos & $4.04(1.681)$ \\
\hline & 3DVMG & $3.88(1.801)$ \\
\hline & 3DVCG & $3.71(1.922)$ \\
\hline \multirow{4}{*}{$\begin{array}{l}\text { Q4 Not sense of being in virtual } \\
\text { space }\end{array}$} & & Mean (SD) \\
\hline & 2DOoS & $3.21(1.444)$ \\
\hline & 3DVMG & $3.08(1.717)$ \\
\hline & 3DVCG & $3.25(1.452)$ \\
\hline \multirow{4}{*}{ Q5 Sense of acting in VE } & & Mean (SD) \\
\hline & 2DOoS & $5.13(1.361)$ \\
\hline & 3DVMG & $5.50(1.022)$ \\
\hline & 3DVCG & $5.33(1.341)$ \\
\hline \multirow{4}{*}{ Q6 Sense of being present in VE } & & Mean (SD) \\
\hline & 2DOoS & $5.21(1.351)$ \\
\hline & 3DVMG & $5.71(0.806)$ \\
\hline & 3DVCG & $5.75(1.073)$ \\
\hline
\end{tabular}

- Involvement

The performances of three interfaces in involvement with the virtual environment were similar, and no significant difference was obtained from results (Figure 39). Most subjects had neutral attitudes to items 7, 9 
and 10 on average in three conditions (most mean values $<5$, see Table 7), and they all felt that they were aware of the real space in all conditions (three mean values $<4, Q 8$ ). It is a reasonable phenomenon since users could not complete their task of avoiding obstacles without the help of safety interfaces, and they would notice the real environment every time when they saw the VSIs. This, as a result, had reduced their involvement with the virtual space and increased the involvement with the real space.

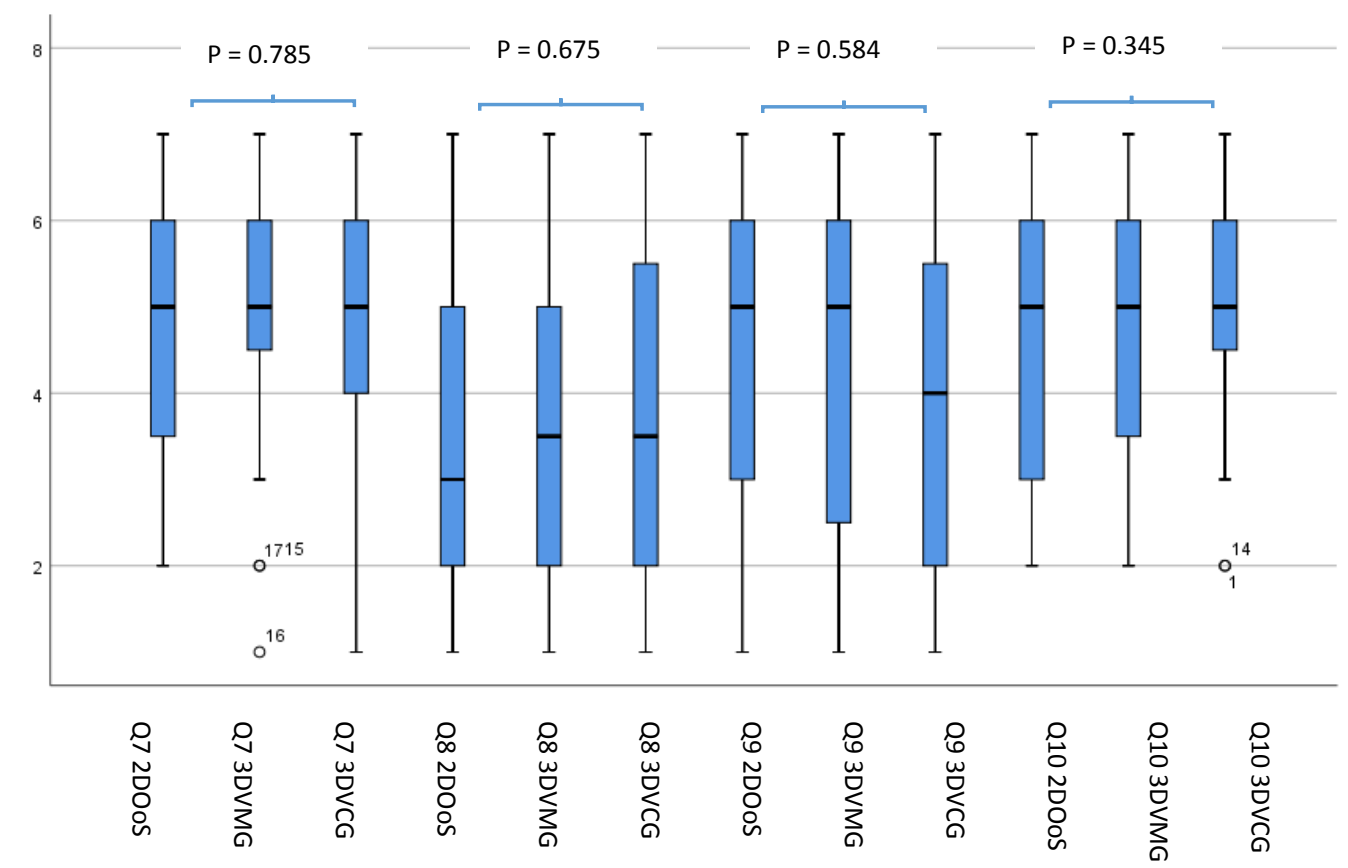

Figure 39 Results of Involvement in Study 1

Table 7 Mean values of involvement in Study 1

\begin{tabular}{|c|c|c|}
\hline \multirow{4}{*}{ Q7 Awareness of real environment } & & Mean (SD) \\
\hline & 2DOoS & $4.67(1.523)$ \\
\hline & 3DVMG & $4.79(1.474)$ \\
\hline & 3DVCG & $4.87(1.624)$ \\
\hline \multirow{4}{*}{ Q8 Not aware of real environment } & & Mean (SD) \\
\hline & 2DOoS & $3.58(1.586)$ \\
\hline & 3DVMG & $3.75(1.751)$ \\
\hline & 3DVCG & $3.75(1.894)$ \\
\hline \multirow{4}{*}{ Q9 Not attention to real environment } & & Mean (SD) \\
\hline & 2DOoS & $4.50(1.745)$ \\
\hline & 3DVMG & $4.29(1.805)$ \\
\hline & 3DVCG & $3.92(1.84)$ \\
\hline \multirow{4}{*}{ Q10 Attention captivated by VE } & & Mean (SD) \\
\hline & 2DOoS & $4.5(1.504)$ \\
\hline & 3DVMG & 4.92 (1.472) \\
\hline & 3DVCG & $5.04(1.488)$ \\
\hline
\end{tabular}


About the realistic level of subjects' virtual experience, there was no significant difference discovered from all four items $(p=0.396, p=0.593, p=0.202$ and $p=0.04$ respectively, as shown in Figure 40 . Generally, according to mean values, the virtual space seemed real to subjects (2DOoS: Mean $=4.67$; 3DVMG: Mean = 5.25; 3DVCG: Mean $=5.21 ; \mathrm{Q} 13)$, and participants had consistent experience between the VE and the real world in all conditions (2DOoS: Mean =4.67; 3DVMG: Mean = 5.08; 3DVCG: Mean = 5.04; Q12). However, the mean values of Q14 showed that subjects gave a lower rating to this question than other questions (all mean values $<4$, see Table 8), which means that respondents refused the statement that the virtual world seemed more realistic than the real world in all conditions. The influential factor for this can be the integrated interfaces presented spatial information of real space. It can be difficult for users to tell which was more realistic, since they always paid attention to real objects and focused on how to avoid the collision.

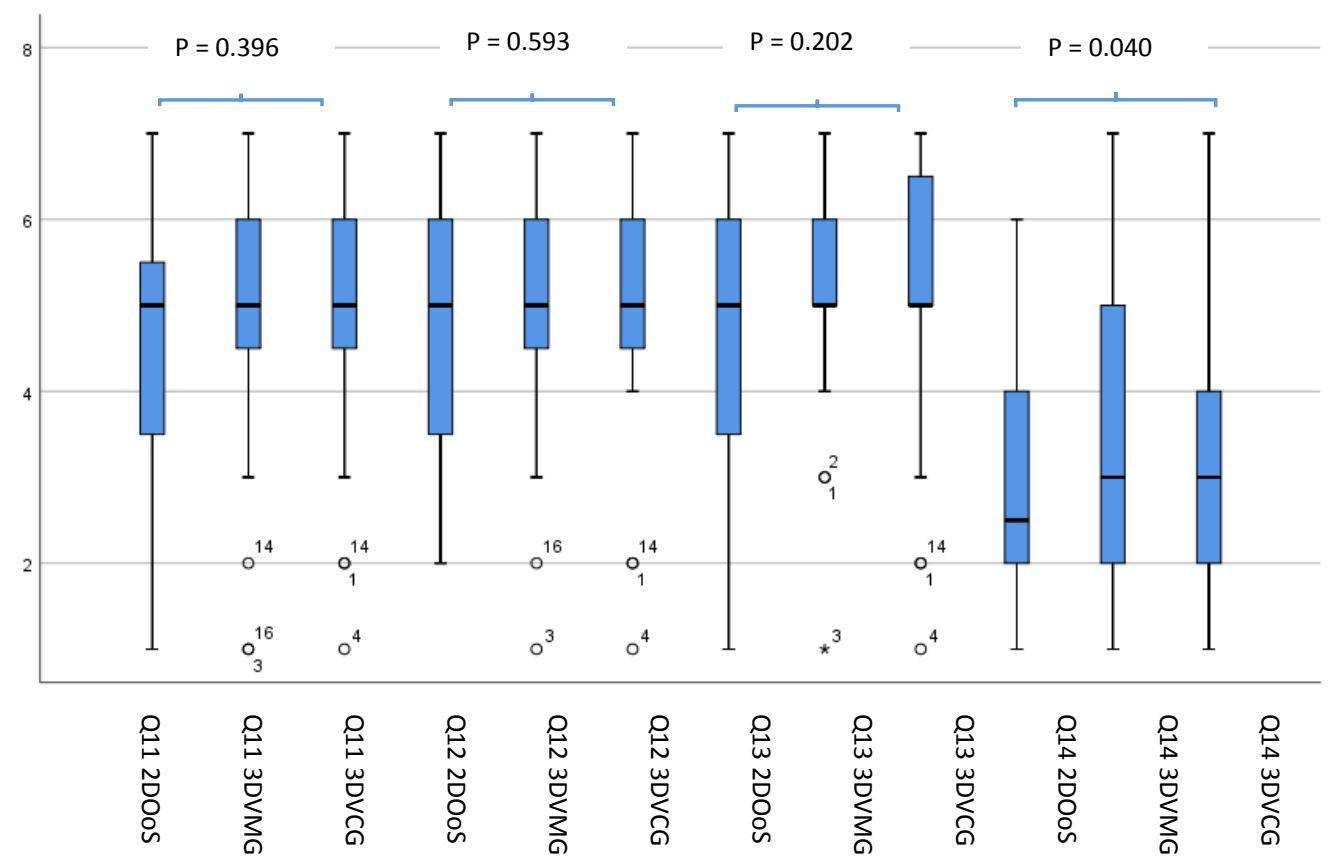

Figure 40 Results of Experienced Realism in Study 1

Table 8 Mean values of Experienced Realism in Study 1

\begin{tabular}{|c|c|c|}
\hline \multirow{4}{*}{ Q11 VE real (real/not real) } & \multicolumn{2}{|r|}{ Mean (SD) } \\
\hline & 2DOos & $4.58(1.501)$ \\
\hline & 3DVMG & 4.67 (1.579) \\
\hline & 3DVCG & $4.92(1.640)$ \\
\hline \multirow{4}{*}{ Q12 Experience similar to real environment } & & Mean (SD) \\
\hline & 2DOos & $4.67(1.373)$ \\
\hline & 3DVMG & 5.08 (1.586) \\
\hline & 3DVCG & 5.04 (1.574) \\
\hline \multirow{2}{*}{ Q13 VE real (imagined/real) } & & Mean (SD) \\
\hline & 2DOos & $4.67(1.523)$ \\
\hline
\end{tabular}




\begin{tabular}{lll}
\hline & 3DVMG & $5.25(1.452)$ \\
\cline { 2 - 3 } & 3DVCG & $5.21(1.693)$ \\
\cline { 2 - 2 } Q14 VE more realistic than the real world & Mean (SD) \\
\cline { 2 - 3 } & 2DOOS & $2.88(1.393)$ \\
\cline { 2 - 3 } & 3DVMG & $3.38(1.610)$ \\
\cline { 2 - 3 } & 3DVCG & $3.42(1.586)$ \\
\hline
\end{tabular}

\subsubsection{Understanding of Physical Layout Information Questionnaire}

Apart from evaluating the immersive effect, respondents evaluated each item after each method at equal intervals via answering a 7-point Likert scales ( 1 for strongly disagree and 7 for strongly agree) questionnaire to measure their understanding of the physical layout information as well (see Table 9), which was adopted from a previous study [29]. Since the acquired data was also ordinal type, a non-parametric test was used to interpret it.

Table 9 Understanding of Real-space Information Questionnaire

\section{Participants could:}

Q1 be aware of the presence of obstacles in the real world

Q2 accurately understand the distance to obstacles

Q3 accurately understand the shape of obstacles

Q4 accurately understand the size of obstacles

Q5 understand the viewing direction in the real world

Q6 could guess their own position in the real world

Q7 precisely predict the location of physical obstacles

Q8 could precisely avoid physical obstacles

Q9 be NOT afraid of suddenly colliding with physical obstacles

Q10 navigate the virtual garden quickly

Q11 felt the real surroundings were represented very well

Q12 felt the appearance of the virtual safety interface was disruptive

Q13 felt mentally stressful

In Study 1 , we applied the Friedman-test $(\alpha=0.05)$ for the data analysis and the Bonferroni Method to adjust the $p$-value which is 0.0167 . Among three VSIs, there were significant differences for using three safety interfaces in regard to participants' confidence in navigating virtual space ( $p=0.009, Q 9$, see Figure 42$)$ and their mental stressfulness ( $p=0.003, Q 13$, see Figure 42). 


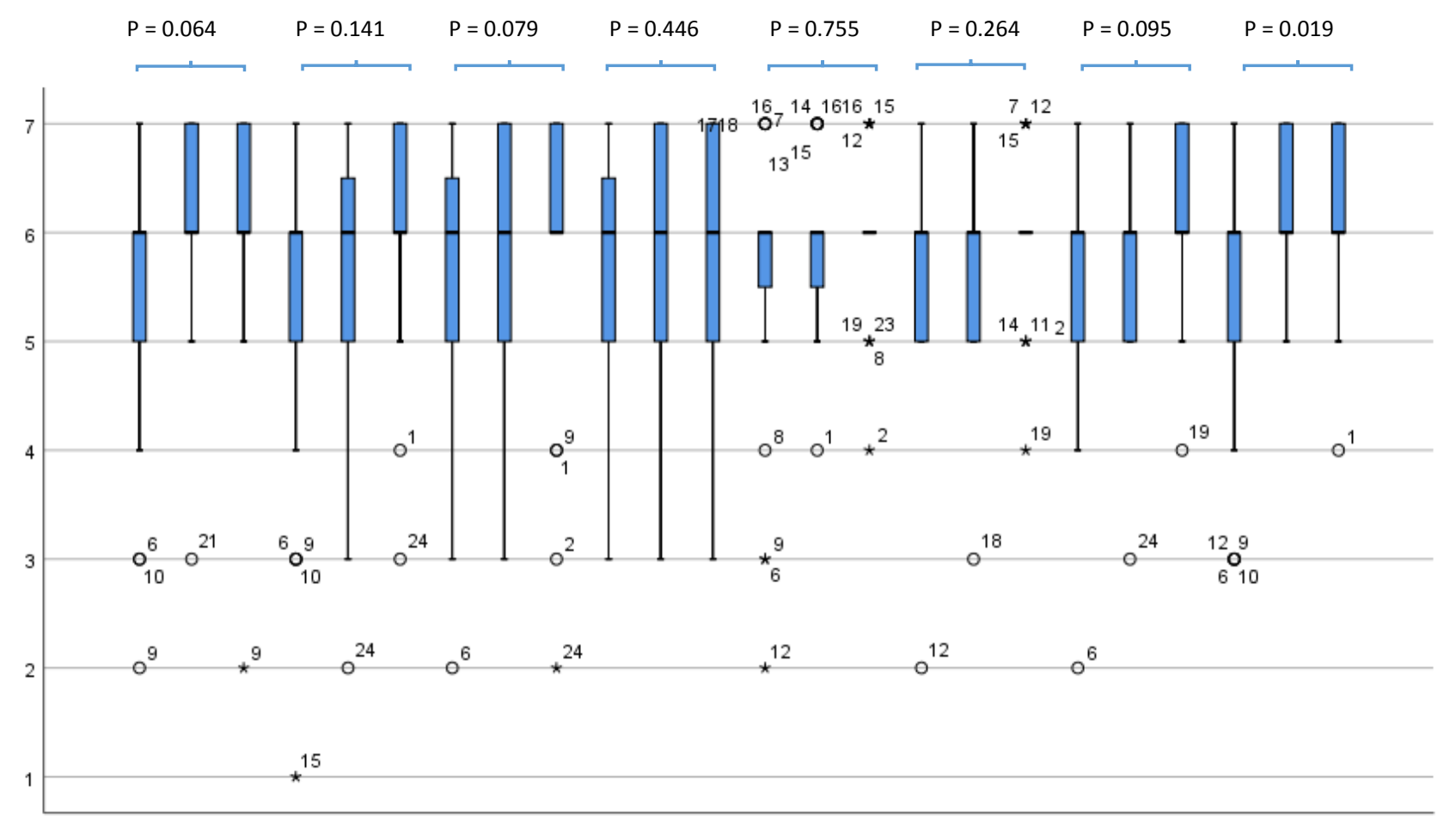

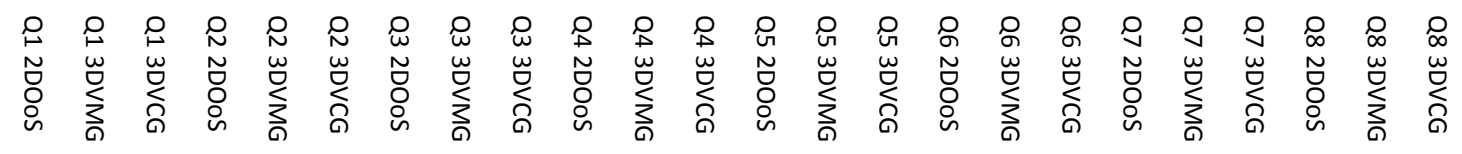

Figure 41 Results of Understanding of Real-space Information in Study 1 (Q1-Q8)

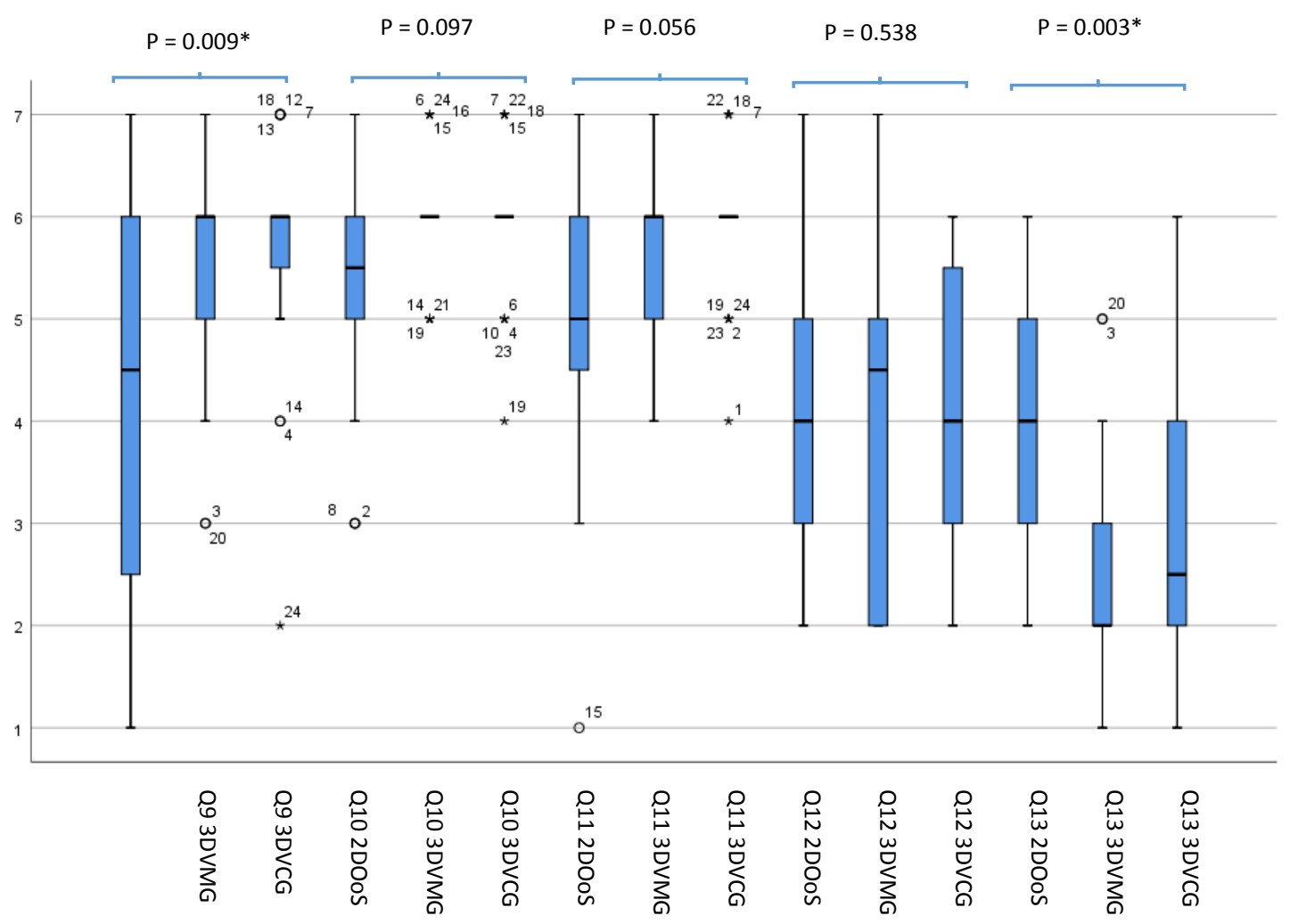

Figure 42 Results of Understanding of Real-space Information in Study 1 (Q9-Q13) 
After further analysing via adopting the Wilcoxon Signed Ranks Test, significant differences between the 2D method and the 3D monochrome interface in $Q 9$ and $Q 13(p=0.009, Q 9 ; p=0.002, Q 13$ respectively, see Figure $43)$, and between the $2 D$ interface and the 3D coloured method in $Q 9(p=0.001, Q 9)$ were confirmed. However, no difference between the 3D monochrome interface and the 3D coloured interface $(p=0.728 Q 9 ; p=0.078$, $Q 13$ respectively) and between 2D interface and 3D coloured method in Q13 $(p=0.039, Q 13)$ was found.

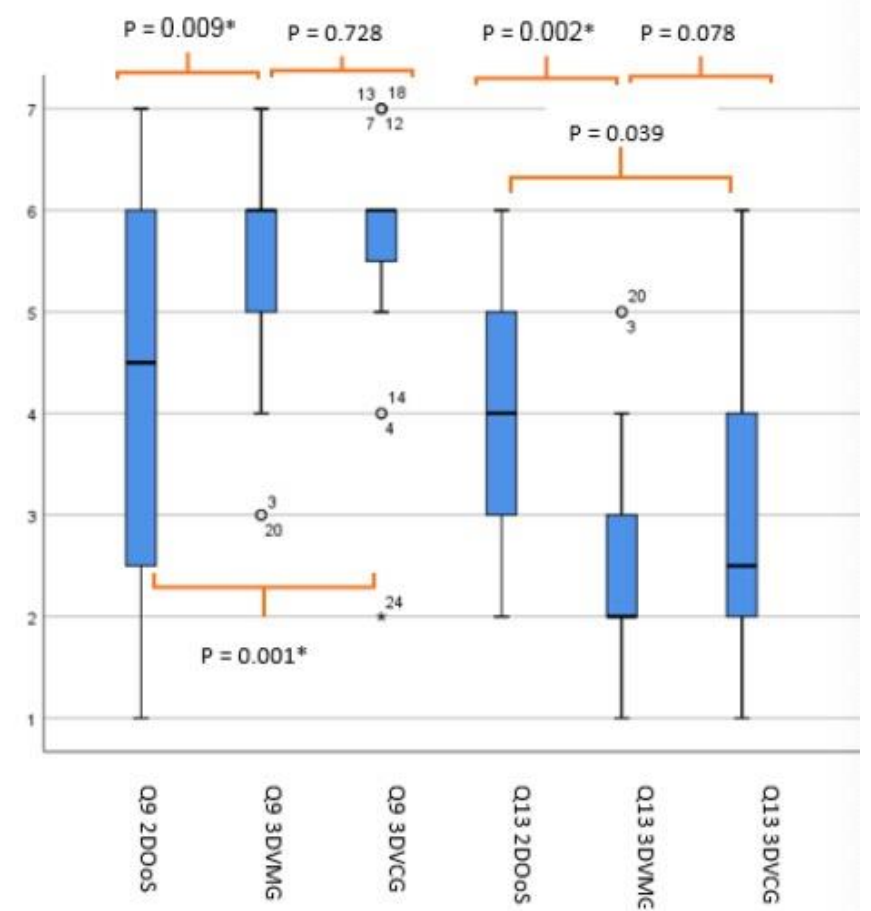

Figure 43 Results of Wilcoxon Signed Ranks Test

Mean values of Q9 shown in Table 10 indicated that subjects can navigate more confidently with two 3D interfaces than 2D Outlines of surroundings (Q9: 2DOoS: Mean $=4.29, \mathrm{SD}=1.899 ; 3 \mathrm{DVMG}$ : Mean $=5.58, \mathrm{SD}$ $=1.176$; 3DVCG: Mean = 5.71, SD = 1.122). For Q13, the mean values indicated that subject experienced less mentally stressful while trying the 3DMG, compared with 2D Outlines (2DOoS: Mean $=3.79, S D=1.285$; 3DVMG: Mean $=2.63, S D=1.135$ ).

Table 10 Results of Understanding of Real-space Information in Study 1

\begin{tabular}{|c|c|c|c|}
\hline & 2D Outlines of Surrounding & $\begin{array}{l}\text { 3D Virtual Monochrome } \\
\text { Grids }\end{array}$ & $\begin{array}{l}\text { 3D Virtual Coloured } \\
\text { Grids }\end{array}$ \\
\hline Q1 & $5.42(1.316)$ & $6.04(0.955)$ & $6.13(1.076)$ \\
\hline Q2 & $5.29(1.517)$ & $5.58(1.283)$ & $6.00(0.978)$ \\
\hline Q3 & $5.58(1.283)$ & $5.71(1.233)$ & $5.92(1.349)$ \\
\hline Q4 & $5.46(1.318)$ & $5.87(1.035)$ & $5.79(1.141)$ \\
\hline Q5 & $5.58(1.316)$ & $5.92(0.776)$ & $6.00(0.722)$ \\
\hline Q6 & $5.63(1.013)$ & $5.62(0.824)$ & $6.00(0.722)$ \\
\hline Q7 & $5.58(1.139)$ & $5.79(0.932)$ & $6.08(0.830)$ \\
\hline Q8 & $5.33(1.308)$ & $6.17(0.761)$ & $6.21(0.779)$ \\
\hline Q9 & $4.29(1.899)$ & $5.58(1.176)$ & $5.71(1.122)$ \\
\hline
\end{tabular}




\begin{tabular}{llll}
\hline Q10 & $5.29(1.197)$ & $5.96(0.624)$ & $5.92(0.717)$ \\
\hline Q11 & $4.96(1.488)$ & $5.58(0.654)$ & $5.88(0.680)$ \\
\hline Q12 & $4.12(1.361)$ & $4.08(1.640)$ & $4.25(1.327)$ \\
\hline Q13 & $3.79(1.285)$ & $2.63(1.135)$ & $3.08(1.381)$
\end{tabular}

About other aspects (Q1-Q8, and Q10 - Q12), there was no significant difference found. However, all these items were rated above 5 on average from Q1 to Q8 and Q10, reflecting that subjects felt they were aware of the presence of obstacles, including the distances to obstacles, the shape and size of real objects, and their viewing direction in all conditions. They could predict positions of themselves and obstacles and then navigate the virtual space quickly and avoid the obstacles precisely with the help of three tested interfaces as well. Participants generally agreed all three interfaces could present the real environment well (Q11: 2DOoS: Mean $=4.96, \mathrm{SD}=1.488$; 3DVMG: Mean = 5.58, SD =0.654; 3DVCG: Mean = 5.88, SD =0.680), while they held a neutral attitude to whether the interface was disruptive or not for all three methods (all mean values $<5, \mathrm{Q} 12$ ).

\subsubsection{Post-experiment Questionnaire}

The post-experiment questionnaire consists of two parts, the preference ranking and their general feedback of each study. The following sections only explain the results of preference ranking, and the feedback will be discussed in Section 5.2.2.

In Study 1, we found the same order of users' preference of four various items, which is 3D Virtual Coloured Grid - 3D Virtual Monochrome Grid - 2D Outlines of Surroundings (3DVCG - 3DVMG - 2DOoS). The followings present the details of each question.

1. For ranking the interfaces in terms of helping to predict the distances to physical objects, $70.83 \%$ participants preferred 3DVCG mostly, $62.5 \%$ of them considered 3DVMG as the second one, and $66.67 \%$ subjects gave the third place to 2DOoS.

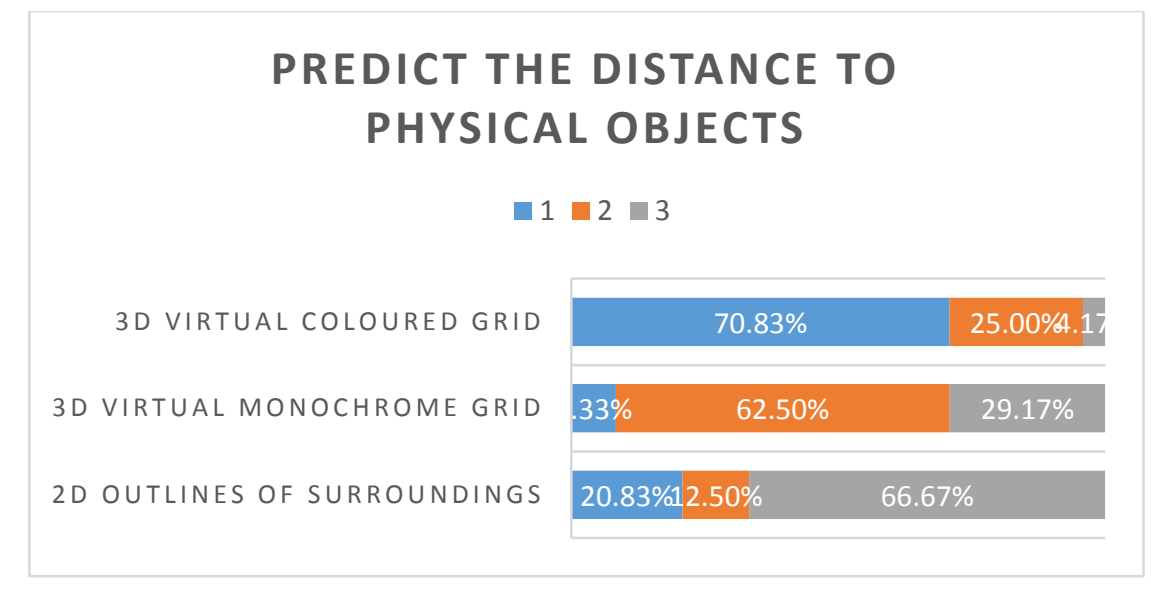

Figure 44 Ranking results of helping to predict the distance to physical objects

2. From the perspective of insisting subjects to avoid obstructions, most (62.65\%) of participants ranked the coloured interface as the best one, before the monochrome one and the 2D Outlines of Surroundings. 


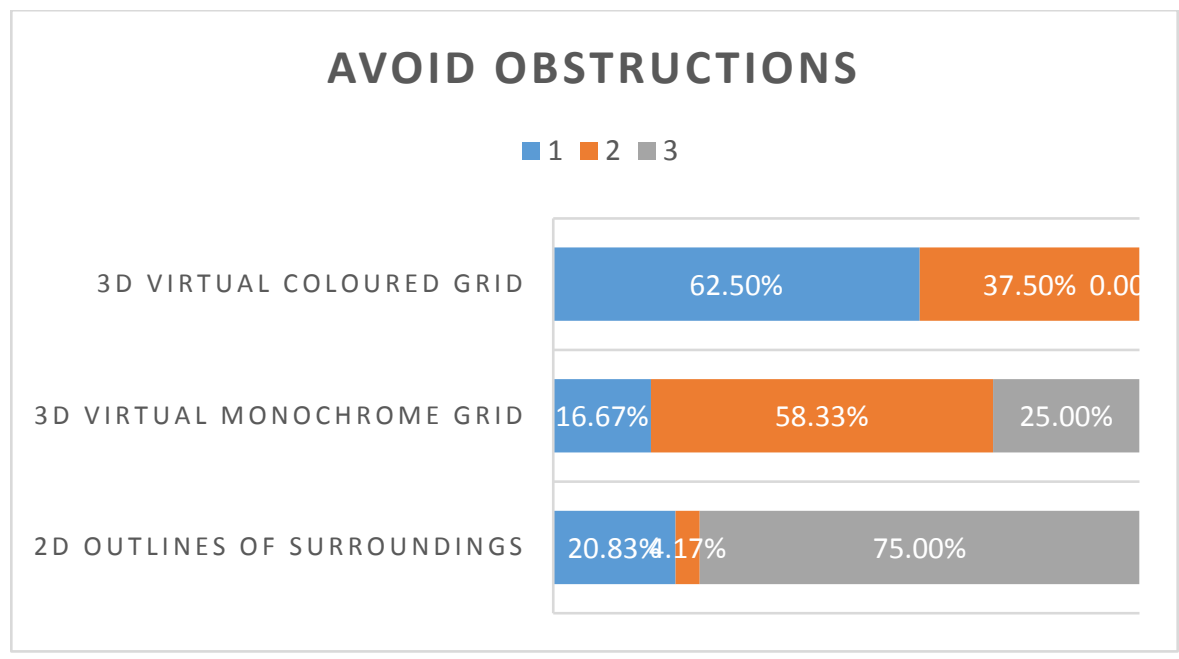

Figure 45 Ranking results of insisting subjects to avoid obstructions

3. Similarly, they felt more confident to navigate the virtual space with the coloured grids than the other two, and the 2D Outlines interface had the worst performance.

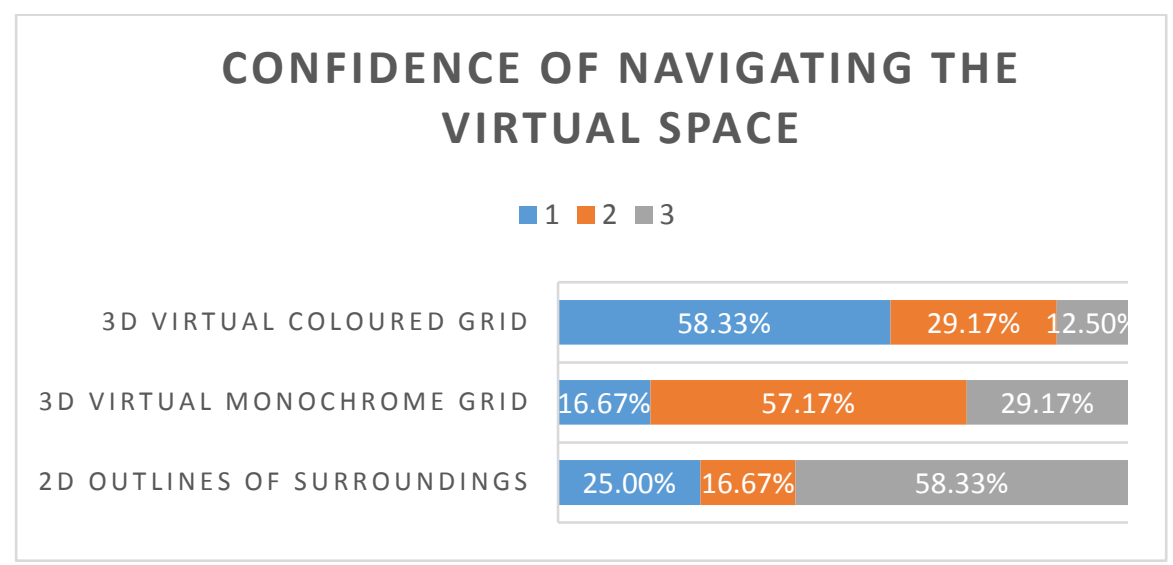

Figure 46 Ranking results of confidence of navigating the virtual space

4. Unsurprisingly, the order of subjects' overall preference among these three interfaces maintained the same order as above.

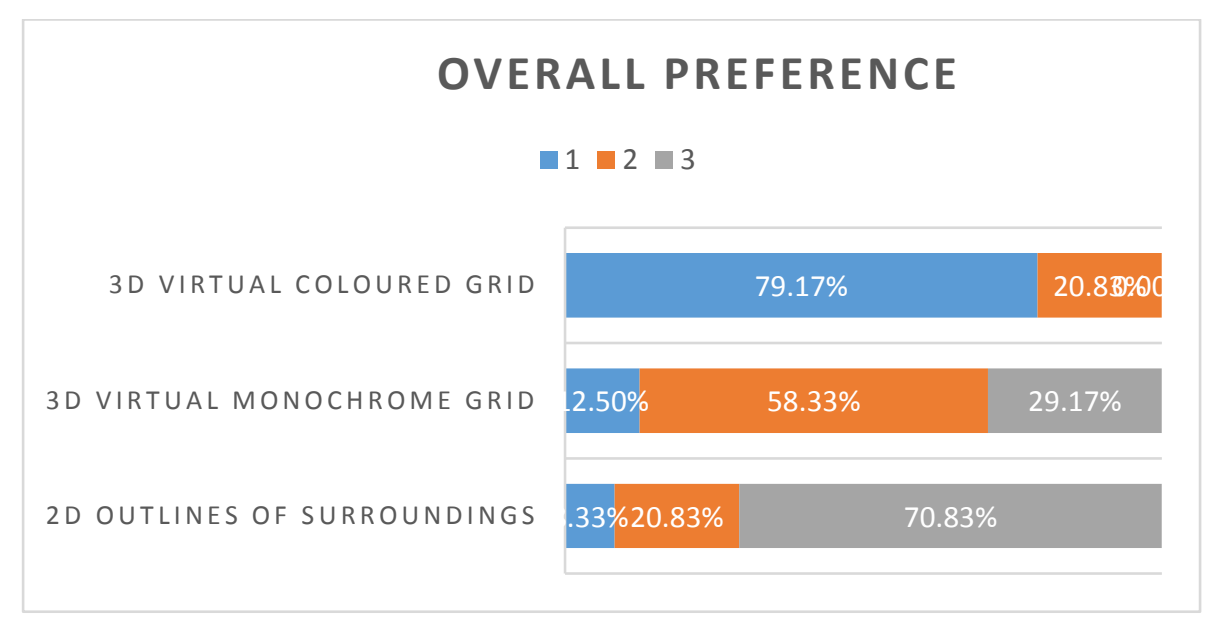

Figure 47 Ranking results of overall preference

\subsubsection{Closest Distance to Obstacles}

We recorded live log data of the participant's movement in each test, all stored as raw data in separate log files, and those data were visualized as path plot images by using the "OriginPro" software. The travel path was used 
as a simple visual reference of each subject, so it is easier for manual evaluation. The Figure 58 is an example that presents users' navigating path in a test as well as positions of two obstacles. The closest distances between obstacles and users were extracted from travel path images, which were used to calculate the significant difference.

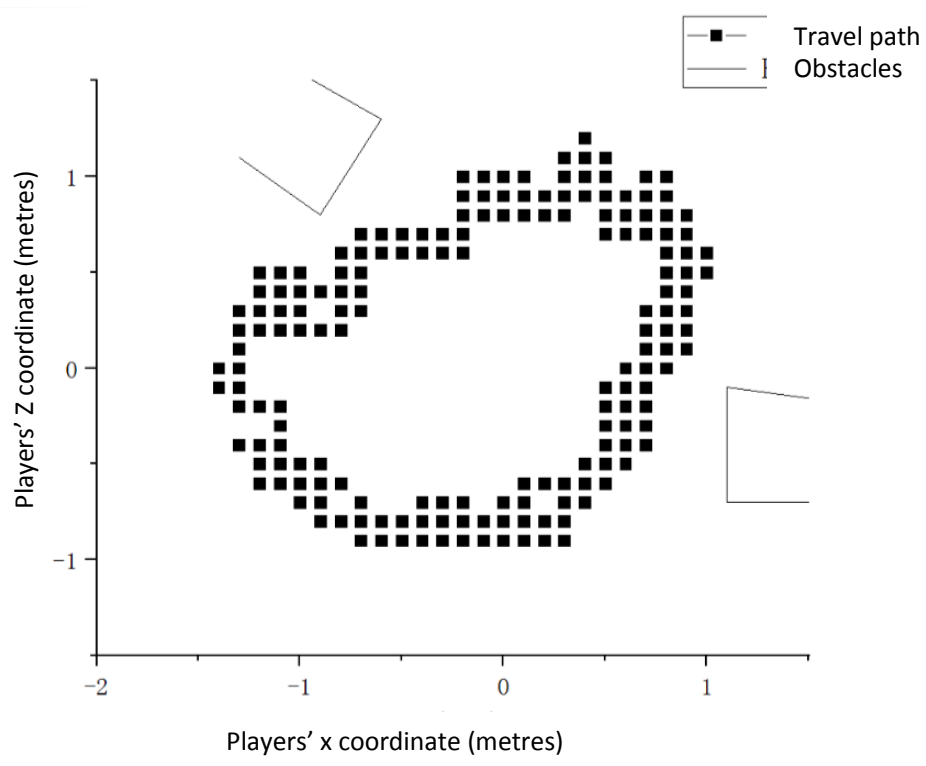

Figure 48 Path Image

In Study 1, to check if there were significant performance differences among three conditions, we first confirmed that the Closest Distances (CDs) to beanbag was normally distributed, but the CDs to the chair was not based on results of the Shapiro-Wilk Test. Therefore, a One-way Repeated Measures ANOVA $(\alpha=0.05)$ was adopted for the former one and a Friedman Test $(\alpha=0.05)$ was used for the latter, and the Bonferroni Method was used to adjust the $p$-value which is 0.0167 . For the CDs to chair (Figure 49), since a significant difference had been found among three items $(P=0.0001)$, Wilcoxon signed rank $(\alpha=0.05)$ test was applied to evaluate the differences between every two items. Greatly significant differences existed between 2D displaying method and 3D monochrome grids, and also between the 2D interface and 3D coloured grids $(p=0.0001, P=0.0001$ respectively), but not existed between two 3D interfaces $(P=0.993)$. Additionally, two 3D interfaces had higher mean distances than that of the 2D interface (2D: Mean $=0.0600, S D=0.0892$; 3DVMG: Mean $=0.3208, S D$ $=0.2677 ;$ 3DVCG: Mean $=0.3033$, SD = 0.2049;). Similar outcomes of CDs to beanbag has found (see Figure 50).

Overall, these data indicated that compared with the 2D safety interface, when subject saw 3D interfaces before they actually stopped or walked away from the obstacles, the reaction distances they needed were shorter. As a consequence, they maintained safer and farther distances to real objects.

Between two 3D interfaces, no significant difference has been found, which means they have similar performances in reminding users of avoiding collisions. 


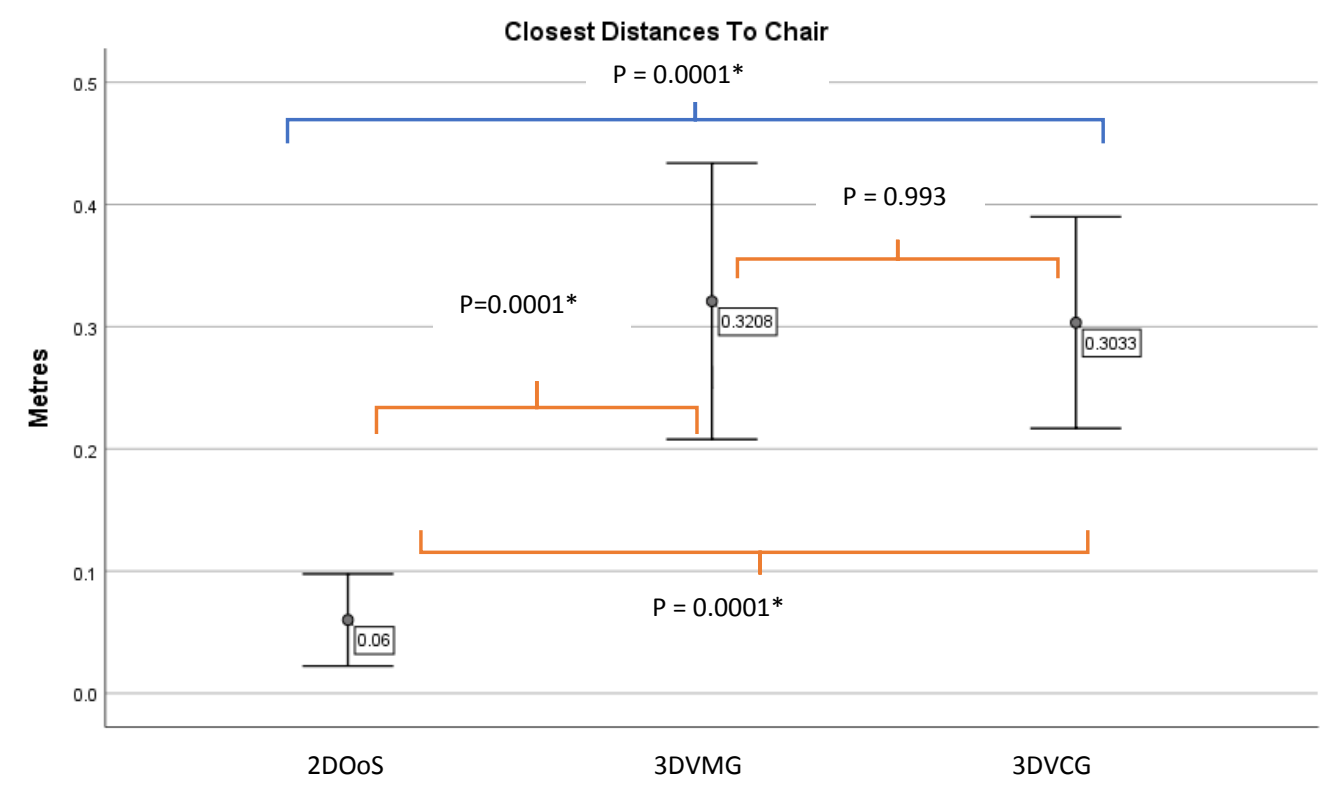

Figure 49 Results of Closest Distances to Chair in Study 1

(*: statistically significant, $\mathbf{0}$ : mean, $\longmapsto:$ : among 3 conditions, $\vdash$ : P between 2 conditions)

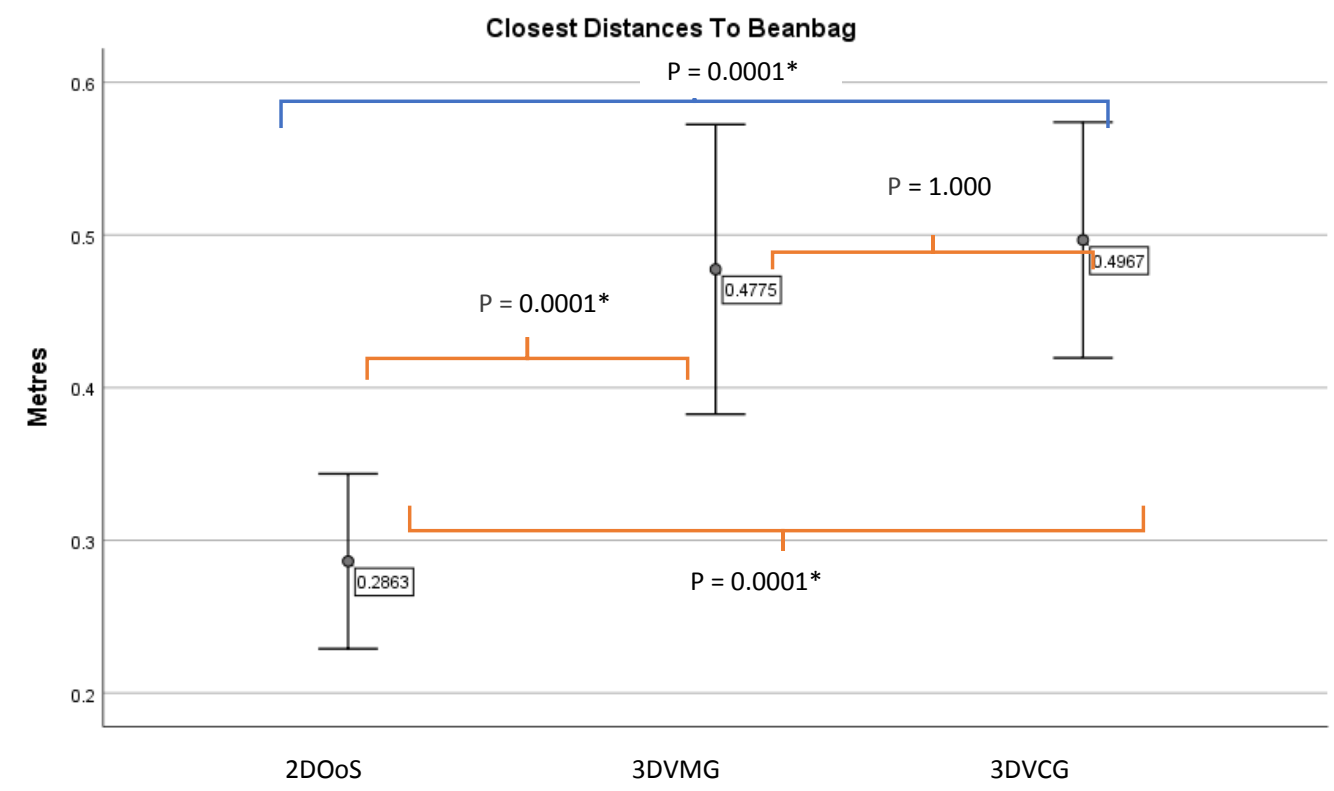

Figure 50 Results of Closest Distances to Beanbag in Study 1

\subsubsection{Collision Count}

During the experiment, the times of subjects colliding with the obstacles were recorded during every condition, which was a determinant and frequently used measure in the previous study either [27] [30] [32].

In Study 1, we found that the collected data did not have normality. Thus, a Friedman Test $(\alpha=0.05)$ was performed among three methods, and we applied the Bonferroni Method to adjust the p-value, which is 0.0167. A significant difference among all items was found ( $p=0.000$, see Figure 51$)$, and then we conducted a Wilcoxon Signed Rank Test to investigate in what way distributions of collision count of the evaluation items were different. As a result, significant differences between the 2D interface and two 3D interfaces respectively were found $(p=0.001, p=0.001$ respectively), except between two 3D interfaces $(p=0.500)$. Great drops of mean 
values between 2D and two 3D methods correspondingly have discovered as well (2D: Mean $=1.2083, S D=$ 1.06237; 3DVMG: Mean =0.0417, SD =0.20412; 3DVCG: Mean =0.0833, SD =0.28233). Therefore, it is obvious that in two novel 3D conditions, subjects were prevented from involving in collisions more successfully than in the $2 \mathrm{D}$ condition.

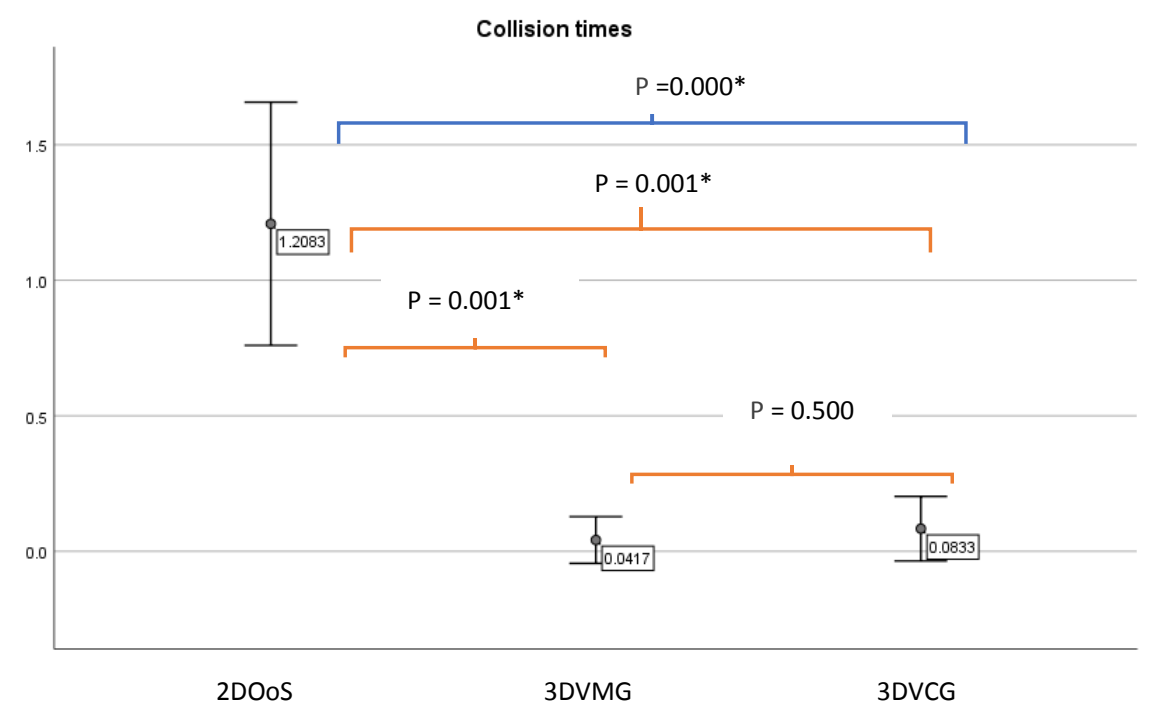

Figure 51 Results of Collision Times in Study 1

\subsubsection{Quantitative Measures Summary}

In Study 1, from the quantitative data of IPQ, there was no significant difference found among three interfaces. We suppose that subjects have a similar experience in terms of presence in all three conditions. The results of understanding of the physical layout information questionnaire show that the 3DVMG had significant impacts on two aspects, "NOT afraid of suddenly colliding with physical obstacles" and "Not felt mentally stressful", while the 3DVCG only had a significant impact on one aspect "NOT afraid of suddenly colliding with physical obstacles". In the post-experiment questionnaire, for all four preference questions, the preferred orders ranked by subjects were 3DVCG-3DVMG-2DOoS. In addition, the results of closest distances measured in path logs presented that significant differences existed between 2DOoS and 3DVMG, 2DOoS and 3DVCG. A similar consequence was found in collision count as well. Compared with two 3D interfaces, users collided with obstacles more frequently in 2DOoS condition.

\subsubsection{Qualitative Measures}

To further understand the reasons behind selections made by respondents during all tests, qualitative measures were also employed in the post-experiment questionnaire by using open-end questions. These questions focused on their responses to reasons for collisions, the general advantages and disadvantages of each method.

In the first open-end question, participants were asked to write down their reasons for colliding with real objects. Here are some of the responses received from the subjects of each method. For the 2DOoS, most subjects complained about its blurry visual style, which resulted in increasing the difficulty in perceiving shapes and positions of the real objects. In addition, they felt that they might need more reaction time to avoid the furniture due to the poor presenting of some obstacles. In terms of 3DVMG, one subject reported that the reconstruction accuracy of this interface should be improved, which was as same as the situation of 3DVCG.

Apart from that, they were asked to describe the advantages and disadvantages of each method, which can contribute to explaining their preferences to some extent. Subjects claimed that with the 2DOoS, the shapes of 
real objects were drawn too simply, they could not understand the entire looks of obstacles or precisely predict the distances to real objects due to the lack of depth information. About $50 \%$ of subjects could not notice it due to its unclear appearance, which also caused uncomfortable feeling if they kept staring at it for a long time. However, they perceived a stronger sense of the existence of obstacles with the help of the two 3D interfaces and made correct decisions to avoid them more quickly. A distinct advantage of 3DVCG reported by many subjects was its colour information allowed them to predict the distances more efficiently, which resulted in a little disruptive to the contrary. These feedbacks aligned well with the outcomes of subjects' preference mentioned in Section 5.2.1.3.

\subsection{Results of Study 2}

\subsubsection{Quantitative Measures}

In Study 2, we had one independent variable (IV), the type of safety interfaces with two levels. The dependent variables were subjects' responses to IPQ, their understanding of the physical layout information questionnaire, their preference, the count they collided with obstacles and the closest distance to obstacles, which was as same as those measured in Study 1. The results of these measurements are described below in details. In our study, an alpha level of 0.05 was used for determining the significant difference.

\subsubsection{Igroup Presence Questionnaire, IPQ}

In Study 2, a statistical method applied to measure data collected from two conditions is the Wilcoxon Signed Rank Test $(\alpha=0.05)$, and we also used the Bonferroni Method to adjust the $p$-value (new $p$-value $=0.025$ ). The results of IPQ are divided into four parts as same as those in Study 1.

- $\quad$ General presence, GP (Q1)

From the data given by respondents, there was no significant difference between two 3D conditions $(p=0.527)$ in terms of sense of being there, but their mean values 5.75 (SD = 1.113) and $5.83(S D=1.049)$ indicated that subjects agreed with having a sense of being there in both conditions.

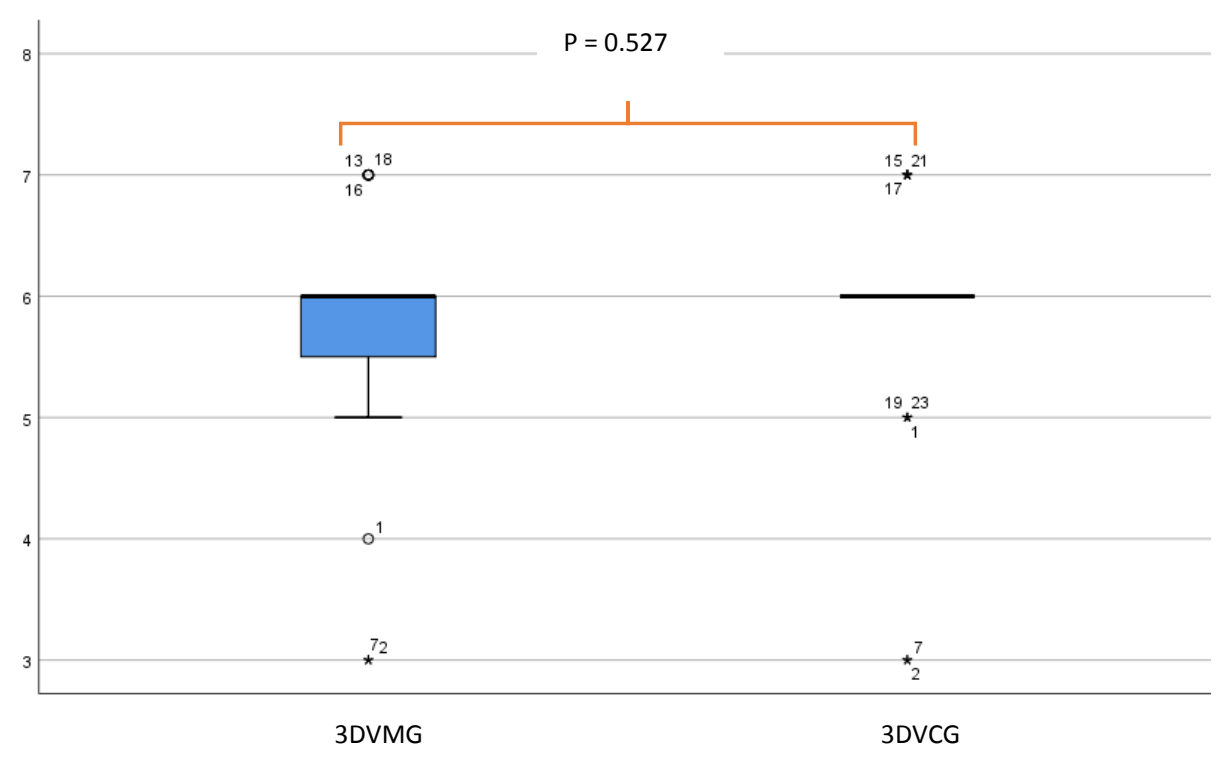

Figure 52 Results of General Presence in Study 2 
Similarly, there was no significant difference found in 5 Spatial Presence items, as shown in Table 11. In regard to sense of VE behind, sense of acting in VE and sense of being present in VE, their mean values demonstrated that subjects agreed with that the tested two interfaces offered spatial presence (all mean values > 5 in Q2, 5 and 6, see Table 11) and disagreed with that they were just watching pictures and not being in the virtual space for two interfaces (all mean values $<4$ in Q3, Q4).

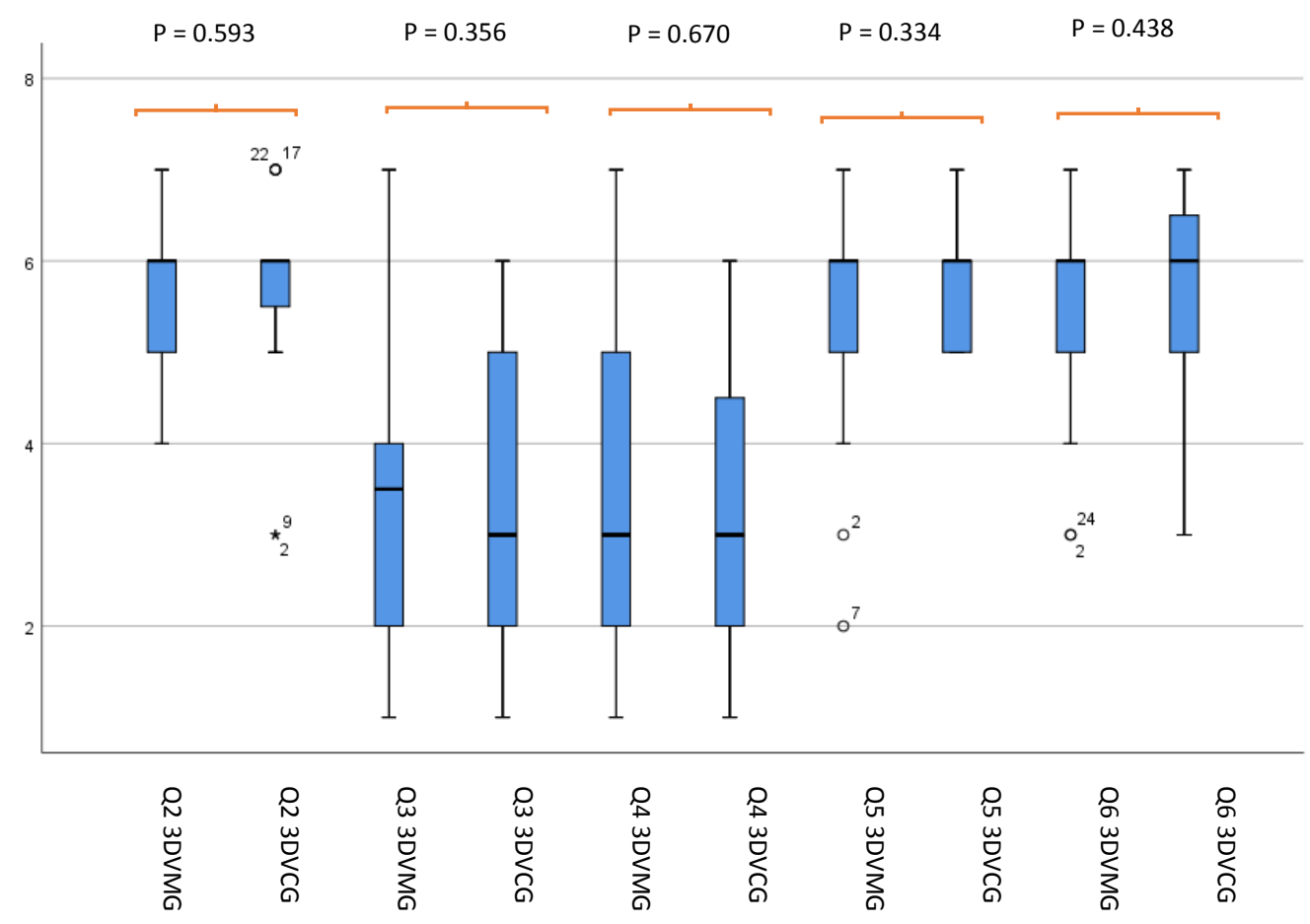

Figure 53 Results of Spatial Presence in Study 2

Table 11 Mean values of Spatial Presence in Study 2

\begin{tabular}{|c|c|c|c|}
\hline \multirow{3}{*}{ Q2 } & \multirow{3}{*}{ Sense of VE behind } & \multicolumn{2}{|r|}{ Mean (SD) } \\
\hline & & 3DVMG & $5.83(0.868)$ \\
\hline & & 3DVCG & $5.71(0.999)$ \\
\hline \multirow{3}{*}{ Q3 } & \multirow{3}{*}{ Only pictures } & & Mean (SD) \\
\hline & & 3DVMG & $3.58(1.666)$ \\
\hline & & 3DVCG & $3.42(1.558)$ \\
\hline \multirow{3}{*}{ Q4 } & \multirow{3}{*}{ No sense of being in virtual space } & & Mean (SD) \\
\hline & & 3DVMG & $3.29(1.628)$ \\
\hline & & 3DVCG & $3.12(1.541)$ \\
\hline \multirow{3}{*}{ Q5 } & \multirow{3}{*}{ Sense of acting in VE } & & Mean (SD) \\
\hline & & 3DVMG & $5.37(1.173)$ \\
\hline & & 3DVCG & $5.63(0.576)$ \\
\hline \multirow{3}{*}{ Q6 } & \multirow{3}{*}{ Sense of being present in VE } & & Mean (SD) \\
\hline & & 3DVMG & $5.54(1.103)$ \\
\hline & & 3DVCG & $5.67(1.239)$ \\
\hline
\end{tabular}


- Involvement

The performances of two interfaces in involvement with the virtual environment were similar in all four aspects, since no significant difference had been found from results as shown in Figure $54(p=0.083, p=0.926, p=0.439$, and $p=0.047$ respectively). Subjects disagreed that they were not aware of the real space (3DVMG: Mean = $5.12, \mathrm{SD}=1.191 ; 3 \mathrm{DVCG}$ Mean $=5.58, \mathrm{SD}=0.881$ respectively for $\mathrm{Q7}$, see Table 12 ) and had neutral attitudes to "no attention to real environment" on average (both mean values $<5, Q 9$ ). It is an unsurprising phenomenon that subjects gave lower than 4 scores for the Q8 which asked whether they were aware of the real environment, since for better guiding users to avoid obstacles, the tested safety interfaces presented detail of the real environment. Every time when subjects saw the interfaces, they would be aware of the real space. Nevertheless, subjects mostly admitted that they were still captivated by the virtual world in both conditions to some degree (3DVMG: Mean $=4.75$, SD = 1.327; 3DVCG: Mean = 5.21, SD = 1.179 respectively for Q10, see Table 12).

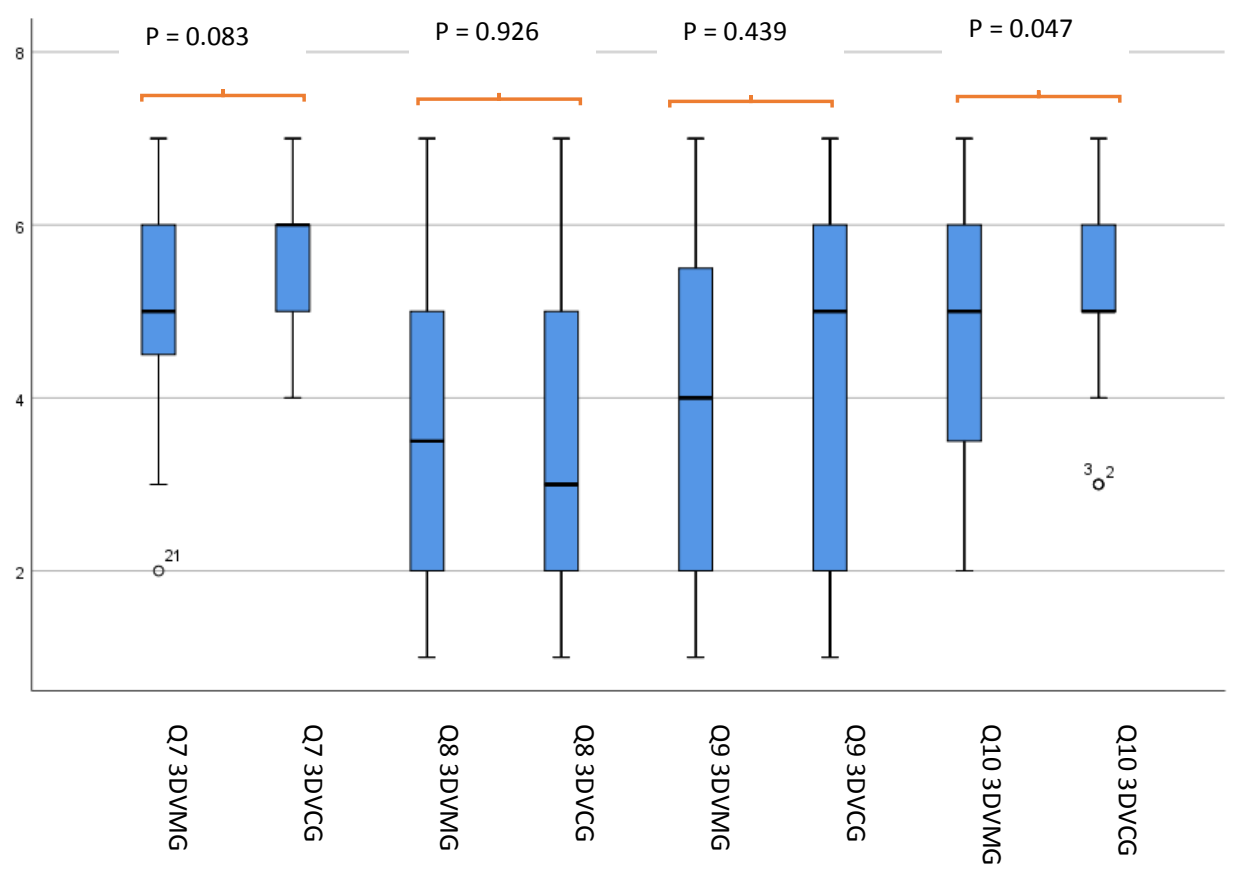

Figure 54 Results of Involvement in Study 2

Table 12 Mean values of Involvement in Study 2

\begin{tabular}{lll}
\hline \multirow{2}{*}{ Q7 Awareness of real environment } & \multicolumn{2}{c}{ Mean (SD) } \\
\cline { 2 - 3 } & 3DVMG & $5.12(1.191)$ \\
\cline { 2 - 3 } Q8 Not aware of real environment & 3DVCG & $5.58(0.881)$ \\
\cline { 2 - 3 } & & Mean (SD) \\
\hline & 3DVMG & $3.63(1.740)$ \\
\hline Q9 Not attention to real environment & 3DVCG & $3.58(1.840)$ \\
\hline & & Mean (SD) \\
\cline { 2 - 3 } Q10 Attention captivated by VE & 3DVMG & $4.00(1.865)$ \\
\hline & 3DVCG & $4.17(1.949)$ \\
\hline & & Mean (SD) \\
\hline
\end{tabular}




\section{- $\quad$ Experienced Realism}

About the realistic level of subjects' virtual experience, there was no significant difference discovered from the first two items ( $p=0.439, p=1.000$, respectively, see Figure 55$)$. The mean values of these two questions indicated that the virtual space seemed real to subjects and they had a consistent experience between the VE and the real world in both conditions. In terms of the last two items, two significant differences were found ( $p$ $=0.013, p=0.009$ respectively). For answering the Q13, subjects considered that the 3D coloured interface maintained the realistic of the VE better (Mean $=5.21, S D=1.351)$, rather than the single-colour one $(m=4.83$, $S D=1.239$ ). For Q14, even though the 3DVCG was rated more highly than the other one, participants considered the VE was less realistic than the real space on average for both conditions (3DVMG: Mean $=3.21, \mathrm{SD}=1.503$; 3DVCG: Mean $=3.88, S D=1.702)$. It means that respondents disagreed that the virtual world seemed more realistic than the real world. The determinant reason can be attributed to the fact that they had to keep the awareness of the real world to avoid obstacles during the experiment, and the VSIs kept reminding them of the real space every time when they approach the obstacles.

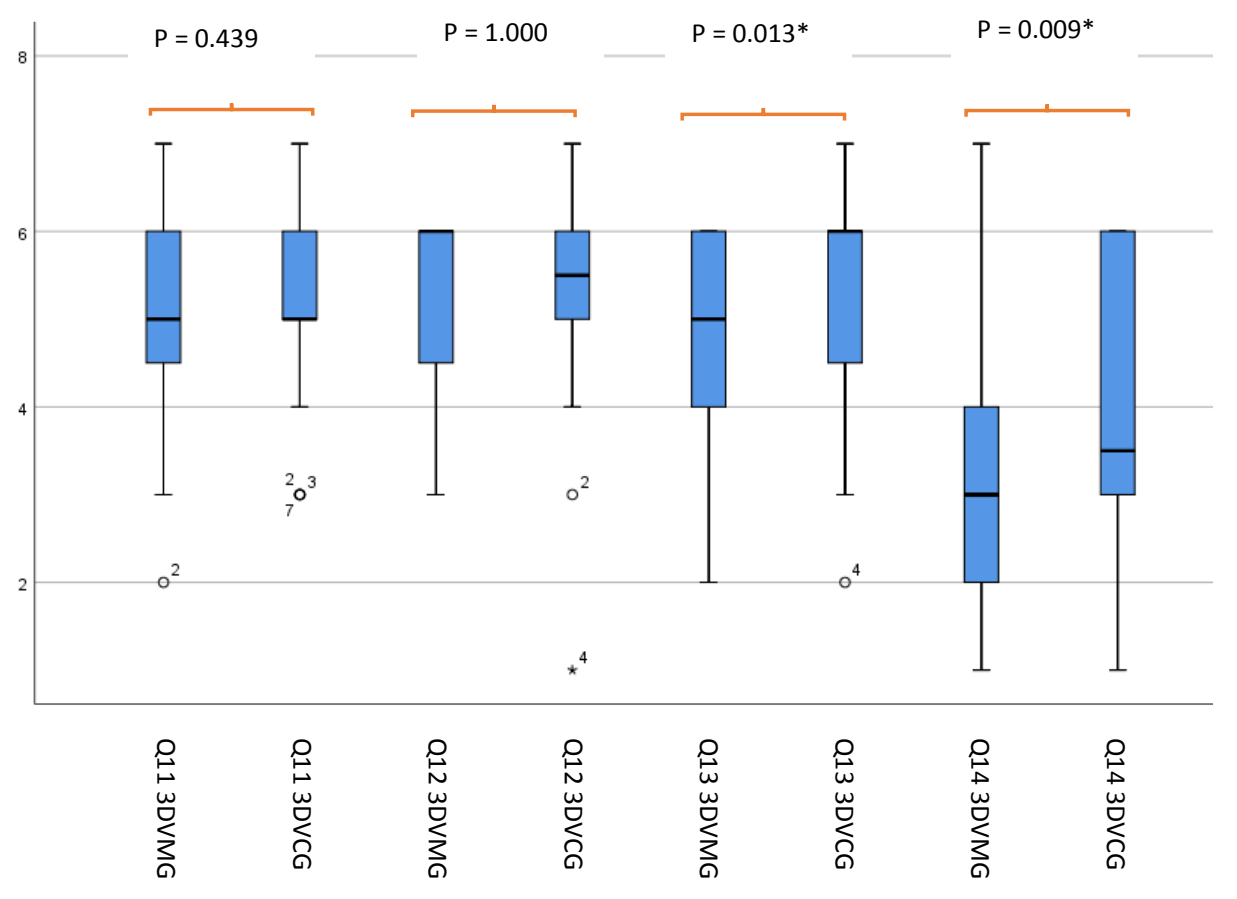

Figure 55 Results of Experienced Realism in Study 2

Table 13 Mean values of Experienced Realism in Study 2

\begin{tabular}{|c|c|c|c|}
\hline \multirow{3}{*}{ Q11 } & \multirow{3}{*}{ VE real (real/not real) } & & Mean (SD) \\
\hline & & 3DVMG & $5.00(1.351)$ \\
\hline & & 3DVCG & $5.12(1.191)$ \\
\hline \multirow{3}{*}{ Q12 } & \multirow{3}{*}{ Experience similar to real environment } & & Mean (SD) \\
\hline & & 3DVMG & $5.21(1.021)$ \\
\hline & & 3DVCG & $5.21(1.318)$ \\
\hline \multirow{2}{*}{ Q13 } & \multirow{2}{*}{ VE real (imagined/real) } & & Mean (SD) \\
\hline & & $3 D V M G$ & $4.83(1.239)$ \\
\hline
\end{tabular}




\begin{tabular}{|c|c|c|c|}
\hline & & 3DVCG & $5.21(1.351)$ \\
\hline \multirow{3}{*}{ Q14 } & \multirow{3}{*}{ VE more realistic than the real world } & & Mean (SD) \\
\hline & & 3DVMG & $3.21(1.503)$ \\
\hline & & 3DVCG & $3.88(1.702)$ \\
\hline
\end{tabular}

\subsubsection{Understanding of Physical Layout Information Questionnaire}

In Study 2, the used understanding of physical layout information questionnaire was the same one used in Study 1. We applied Wilcoxon Signed Ranks $(\alpha=0.05)$ Test to analyse the difference between two 3D VSIs, and we also used the Bonferroni Method to adjust the $p$-value (new $p$-value $=0.025$ ). Significant differences existed in terms of their ability to understand the distances to obstacles $(p=0.000, Q 2$, see Figure 56$)$, and their capacity for avoiding obstacles ( $p=0.0045, Q 8$, see Figure 57$)$.

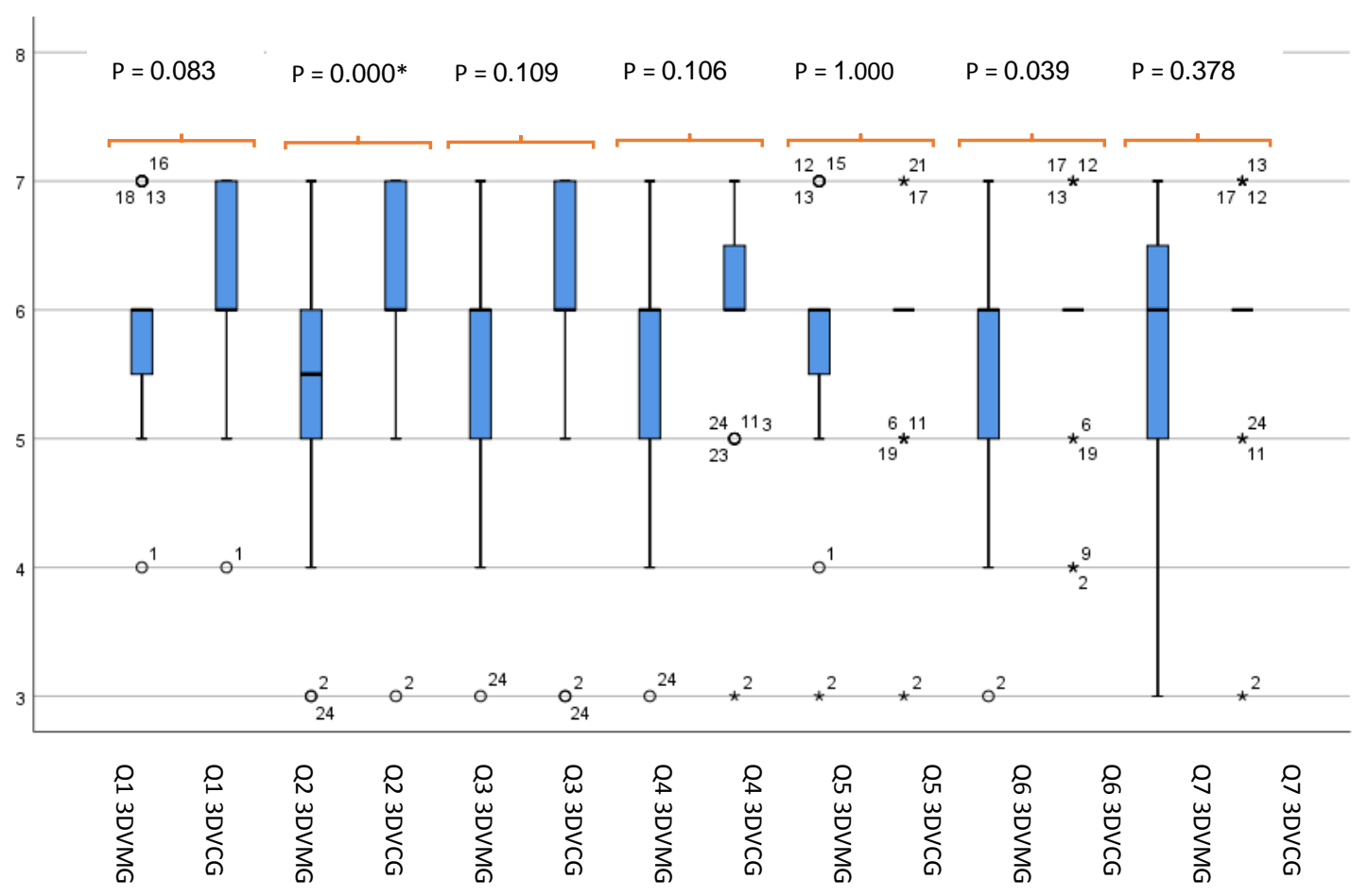

Figure 56 Results of Understanding of Real-space Information in Study 2 (Q1 -7) 


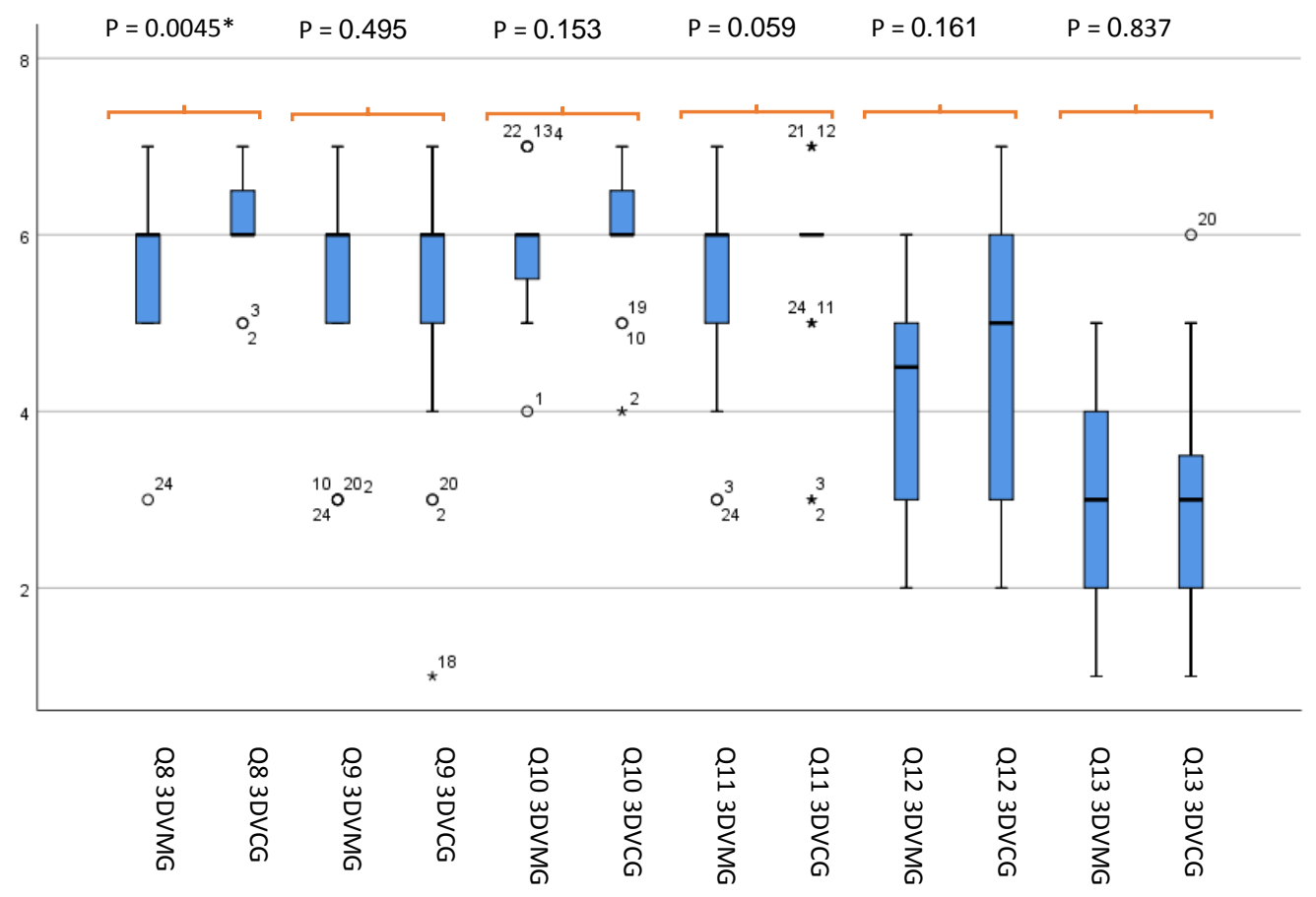

Figure 57 Results of Understanding of Real-space Information in Study 2 (Q8 -13)

Combined with the results of mean values(as shown in Table 14) and $p$ values as shown in the above pictures, a conclusion can be drawn that compared to the 3D single-colour interface, the multiple-colours one helped participants understanding distances to obstacles, and avoiding collisions significantly better (Q2:3DVMG: Mean= 5.33, SD =0.963; 3DVCG: Mean=6.04, SD = 0.955; Q8: 3DVMG: Mean = 5.67, SD =0.816; 3DVCG: Mean $=6.17, \mathrm{SD}=0.565)$.

Table 14 Results of Understanding of Real-space information in Study 2

\begin{tabular}{lll}
\hline & 3D Virtual Monochrome Grids & 3D Virtual Coloured Grids \\
\hline $\mathbf{1}$ & $5.92(0.776)$ & $6.17(0.761)$ \\
\hline $\mathbf{3}$ & $5.33(0.963)$ & $6.04(0.955)$ \\
\hline $\mathbf{4}$ & $5.67(0.963)$ & $5.92(1.100)$ \\
\hline $\mathbf{5}$ & $5.67(1.007)$ & $5.96(0.908)$ \\
\hline $\mathbf{6}$ & $5.79(0.932)$ & $5.79(0.779)$ \\
\hline $\mathbf{7}$ & $5.54(0.884)$ & $5.92(0.776)$ \\
\hline $\mathbf{8}$ & $5.83(0.963)$ & $6.00(0.834)$ \\
\hline $\mathbf{9}$ & $5.67(0.816)$ & $6.17(0.565)$ \\
\hline $\mathbf{1 0}$ & $5.42(1.248)$ & $5.54(1.474)$ \\
\hline $\mathbf{1 1}$ & $5.83(0.702)$ & $6.08(0.717)$ \\
\hline $\mathbf{1 2}$ & $5.42(1.060)$ & $5.75(0.989)$ \\
\hline & $4.25(1.359)$ & $4.50(1.719)$ \\
\hline $2.79(1.318)$ & $2.83(1.404)$ \\
\hline
\end{tabular}


About other aspects (Q1, Q3-Q7 and Q9-Q13), there was no significant difference found. Nevertheless, the mean values for item 1 to item 11 were all higher than five, stating that subjects' attitudes to these questions were positive for both interfaces. Subjects felt they were aware of the presence of obstacles, the shape and size of real objects, their viewing direction, could predict positions of themselves and obstacles and then could navigate the virtual space quickly without fear, and felt the real environment was presented well for both conditions. Subject had a neutral attitude to whether the two interfaces were disruptive (Q12: both means < 5) and they disagreed that the two interfaces had caused uncomfortable feelings (Q13: both means <3).

\subsubsection{Post-experiment Questionnaire}

The post-experiment questionnaire used in Study 2 was the same one used in Study 1. The following sections only explain the results of preference ranking, and the feedback is discussed in Section 5.3.2.

We collected the same order of users' preferences of four various items, which is 3D Virtual Coloured Grid - 3D Virtual Monochrome Grid. Firstly, for ranking the interfaces in terms of helping to predict the distances to physical objects, 92\% participants preferred Coloured 3D Grids mostly, and the rest of them considered 3D Virtual Monochrome Grid as the second one (Figure 58). Similarly, from the perspective of insisting subjects to avoid obstructions, most (96\%) of them ranked the coloured interface as the best one, before the monochrome one (Figure 59). Subjects also felt more confident while navigating the virtual space under the help of the coloured grid than the other (Figure 60). Overall, the coloured interface was unsurprisingly considered as a superior one by $92 \%$ subjects (Figure 61).

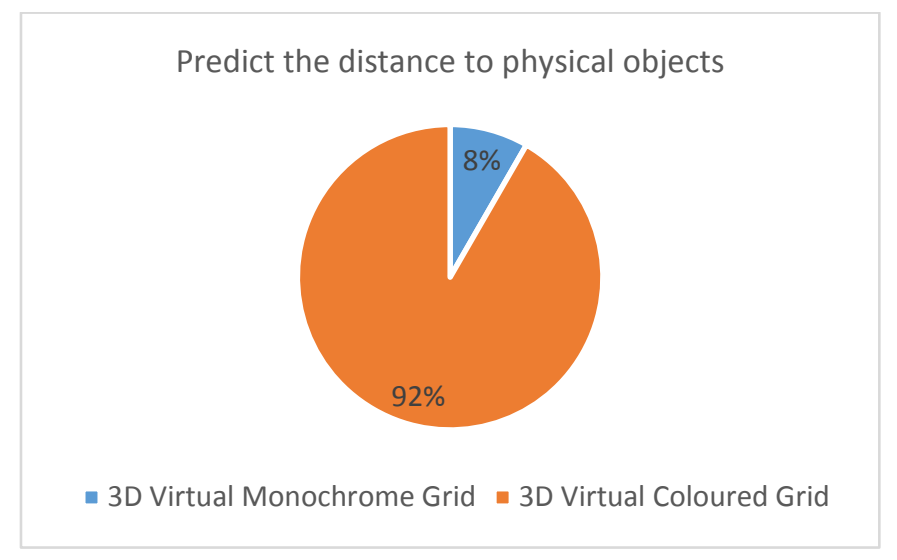

Figure 58 Ranking results of helping to predict the distance to physical objects

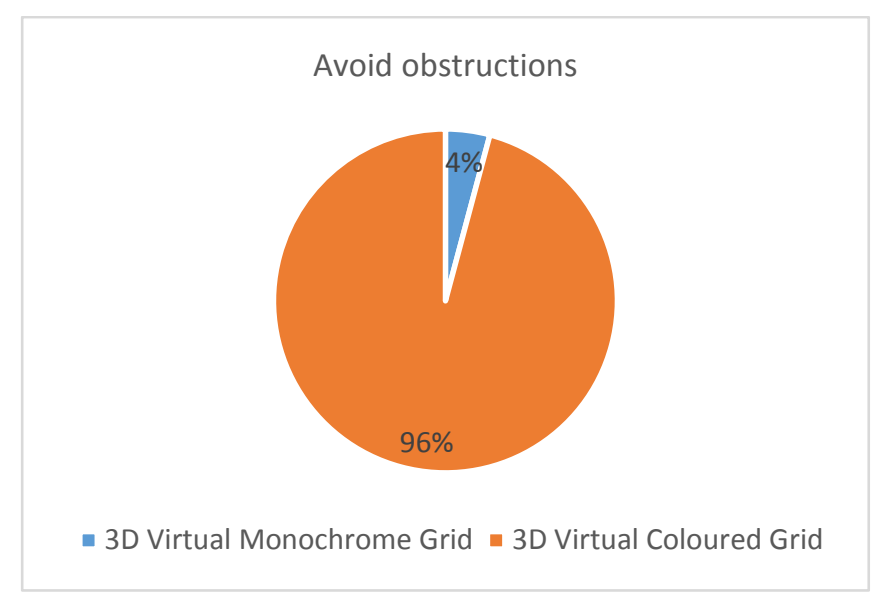

Figure 59 Ranking results of insisting subjects to avoid obstructions 


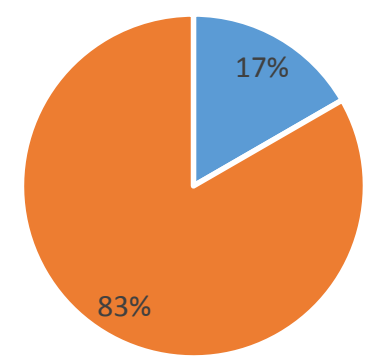

- 3D Virtual Monochrome Grid - 3D Virtual Coloured Grid

Figure 60 Ranking results of confidence of navigating the virtual space

Overall preference

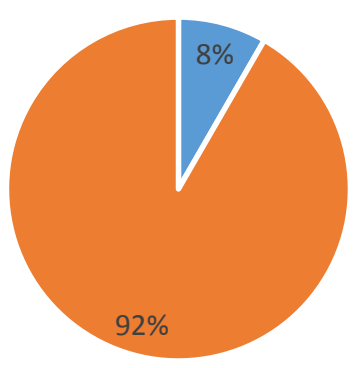

- 3D Virtual Monochrome Grid $=3 \mathrm{D}$ Virtual Coloured Grid

Figure 61 Ranking results of overall preference

\subsubsection{Closest Distance}

In Study 2, the closest distances were measured in the same way as which used in the Study 1. We found that the collected distances of CDs to chair violating the normal distribution, while CDs to beanbag having normality was confirmed via performing a Shapiro-Wilk Test. To investigate how distributions of closest distances of evaluation items were different, we correspondingly performed the Wilcoxon Signed Rank Test $(\alpha=0.05)$ and Paired-Samples T-tests $(\alpha=0.05)$ between each pair of conditions for each set of evaluation results. We also used the Bonferroni Method to adjust the $p$-value (new $p$-value $=0.025$ ). In the following figures, the $p$ values indicated that there was no significant difference between two 3D safety interfaces $(p=0.059, p=0.383$, respectively). Since in this test, participants were required to maintain very close distances to obstacles in both conditions, it is unsurprising to see this outcome. 


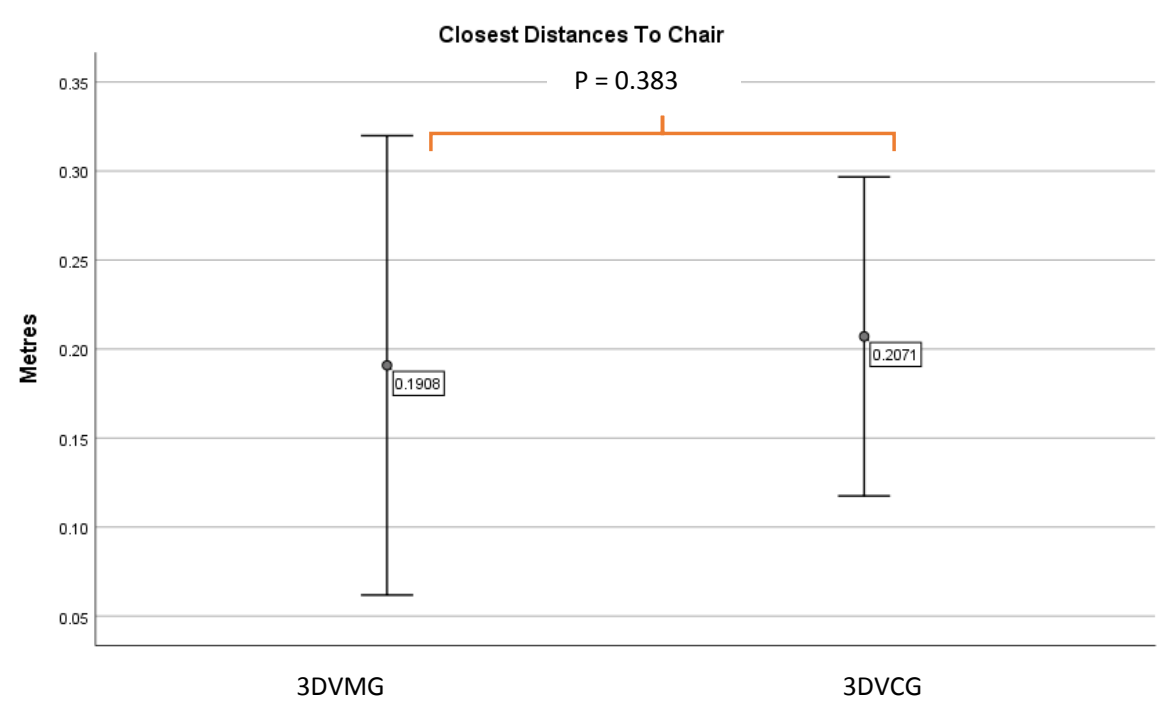

Figure 62 Results of Closest Distances to Chair in Study 2

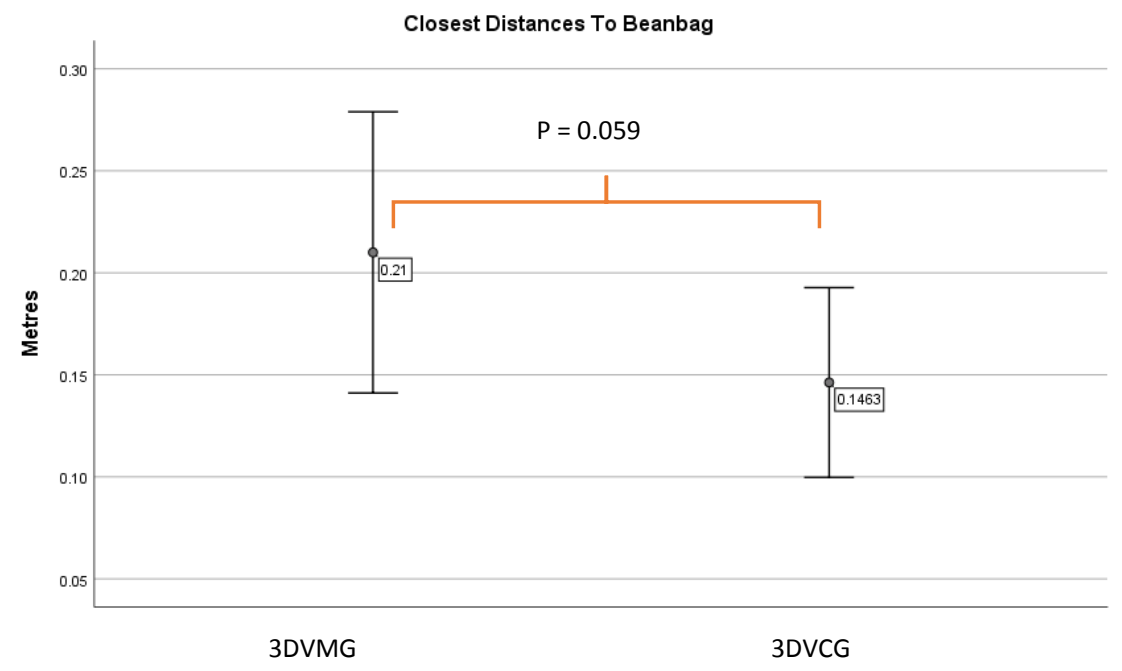

Figure 63 Results of Closest Distances to Beanbag in Study 2

\subsubsection{Collision Count}

In Study 2, we collected the collision count as well. There was a significant difference between the 3D Virtual Monochrome Grid and the 3D Coloured Grids ( $p=0.006$, see Figure 64$)$ by performing a Wilcoxon Signed Rank Test $(\alpha=0.05)$. The Bonferroni Method was applied to adjust the $p$-value (new $p$-value $=0.025$ ). The results shown in the following figure also demonstrated that the mean of the single colour interface is higher than that of the multiple-colour one (3DVMG: Mean $=1.2083, \mathrm{SD}=1.31807$; 3DVCG: Mean $=0.4167, \mathrm{SD}=0.65386$, respectively). Hence, compared with the monochrome interface, the usage of multiple colours to interpret different distances can offer more clues to users for collision avoidance purpose when they stand in a close place to objects in the real space. 


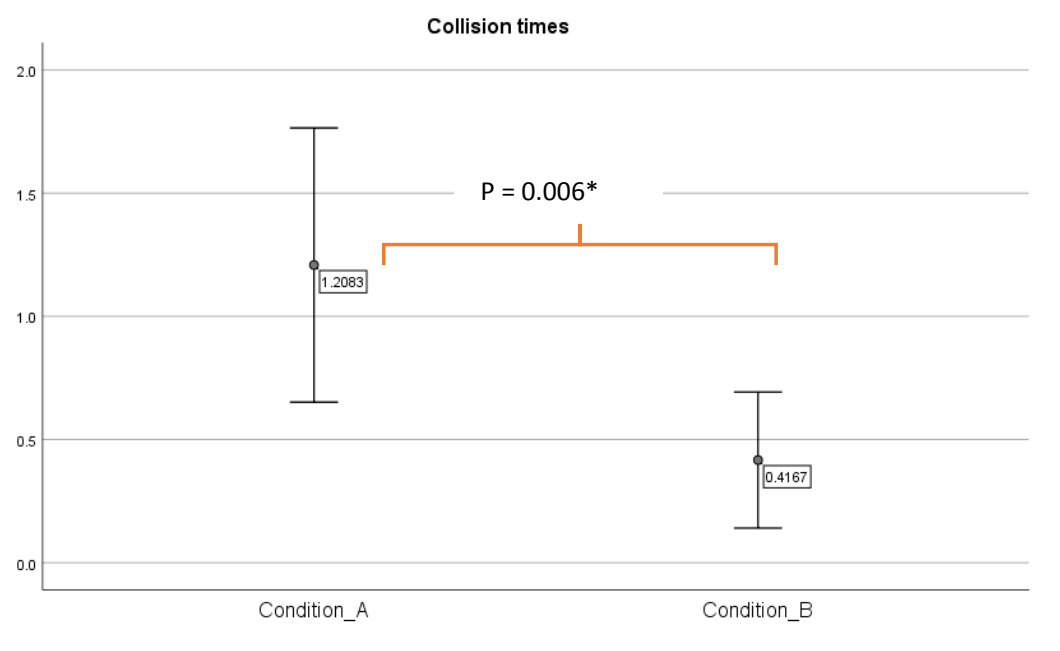

Figure 64 Results of Collision Times in Study 1

\subsubsection{Quantitative Measures Summary}

In Study 2, the quantitative data of IPQ showed that 3DVCG had significant influences on experienced realism ("VE is real" and "VE is more realistic than the real world"). Therefore, in this aspect, subjects had more immersive experiences in the 3DVCG condition than in the 3DVMG condition, under a circumstance in which users were asked to stay near to obstacles. We also compared the results of IPQ collected in Study 1 and Study 2 , and we found that there was no great difference because the two 3D interfaces were given similar mean values by subjects in both studies. It means that subjects had a similar experience of immersiveness, although they were in two different scenarios. The results of understanding of the physical layout information questionnaire show that the 3DVCG had significant impacts on two items, "Accurately understood the distance to obstacles", and "Could precisely avoid physical obstacles". In the post-experiment questionnaire, the preferred orders ranked by subjects are 3DVCG-3DVMG for all four preference questions. Apart from that, a significant difference was found in collision count between two 3D interfaces, and subjects avoided collisions more successfully in 3DVCG condition than the other one.

\subsubsection{Qualitative Measures}

In Study 2, participants were asked to answer the same open-ended questions as they did in the Study 1. Based on the qualitative data, the reason behind participants bumping into obstacles more frequently in 3D single colour mode was they could not estimate the distances to obstacles very precisely (in Centimetre) when they walked too close to the objects in 3DVMG condition. This concern was reported in the second condition as well, but the difference was that fewer people encountered with this issue. The essential factor for this can be compared with the 3DVMG the multicolour gave more precaution for users to remind them of the distances.

About the responses to the advantages and disadvantages of each method, overall subjects explained that it was still difficult for them to precisely predict the distances to obstacles under the help of two 3D interfaces, while they came too close to the objects. They expected that the detail of edges of real objects could be rendered more accurately. Generally, they complimented the excellent performance of two 3D interfaces in outlining the real obstacles, which led them to avoid the physical objects quickly. Last but not least, participants preferred 3DVCG over 3DVMG as the colour clue improved the safety precaution, and then they could judge which object was dangerous and which was not and distances to obstacles from colours with more clues, and 
then make better decisions. Similarly, they presented their expectation of a more straightforward rendering style of two 3D interfaces, and some small details of real objects can be drawn more precisely. 


\section{Chapter 6 Discussion}

This chapter discusses the results found in our user studies. It also explores possible explanations of the results and the limitations of the studies.

\subsection{Discussion of Study Results}

\subsubsection{Users' Ability to Perceive the Obstacles in Study 1}

In Study 1, we compared three interfaces in a typical VR room-scale playing scenario for investigating the usability of our proposed 3D interfaces in this situation. Subjects were asked to navigate the virtual garden by following some virtual paths and avoid obstacles in the real environment with the help the different interfaces. We place one chair and one beanbag outside the boundaries as required in a VR room-scale mode to provide a fair experimental environment for three conditions.

The results of understanding of the physical layout information questionnaire showed that a significant difference between the 2D method and two 3D interfaces respectively were confirmed in one aspect, which is "NOT afraid of suddenly colliding with physical obstacles". Subjects considered that they had more confidence to navigate in the VE without worry about suddenly bumping into real objects in two 3D conditions rather than in the $2 \mathrm{D}$ condition. Combined with the analysis of subjects' feedback, we think there are some contributing factors. Firstly, in 2DOoS condition, the blurry visual style and the poor presenting of some obstacles can reduce subjects' ability to perceive shapes and positions of real objects. Apart from that, the 2DOoS lacks depth information, so subjects cannot predict the distances between obstacles and themselves very precisely. However, they perceived a stronger sense of the existence of obstacles with the help of two 3D interfaces. Thus, subjects can avoid obstacles as quickly as they can whenever they see the warning interfaces.

The fact that 3D interfaces offer more warning information for users to alert them to maintain safer distances to obstacles has been proved by the results of the closest distances since significant differences existed between 2DOoS and 3DVMG, and between 2DOoS and 3DVCG respectively. From our observation and subjects' verbal feedback, compared with the 2D interface, subjects avoided real objects in further distances in advance in two $3 \mathrm{D}$ conditions. However, when they saw 2DOoS, they needed more time to deal with the spatial information offered by this interface, such as distances to real objects and positions of obstacles. This result clarified that 3D interfaces had help subjects in perceiving obstructions better, so they could walk away from real objects faster. However, when considering the available space, the result that the users keep further distances between themselves and obstacles can be a drawback on the other hand, since the usable space can be reduced in this situation the extent to which.

Besides, the outperformance of two 3D interfaces in guiding subjects to navigate the VE more confidently was found in the collision count as well, as the numbers of collisions in two 3D conditions were much less than that of the 2D interface. As mentioned above, subjects maintained further distances between themselves and obstacles in two 3D conditions, which can result in less possibility that they will bump into objects in the real environment. Therefore, smaller collision counts were obtained in these two conditions.

The orders ranked by subjects of their preference of these three interfaces are 3DVCG-3DVMG-2DOoS (from the best to the worst), which is as the same as our expectation. We think it is a reasonable outcome according to the results of used measurements including the physical layout information questionnaire, collision count and the closest distances. 
Therefore, the overall results of these measures show that compared with 2DOoS, users' ability to perceive the obstacles had been improved with the help of both 3D interfaces.

Another significant difference between the 2DOoS and the 3DVMG was found in the item "felt mentally stressful". According to the comments given by subjects, one determining reason can be attributed to the unclear visual style of the 2D interface which causes stressfulness of subjects' eyes when they stare at the interface for a long time. However, unlike our expectation, a significant difference between the 2DOoS and the 3DVCG in this aspect was not discovered as some subjects reported that the multiple colours of the 3DVCG distracted them the extent to which, even though it had superior warning function. Therefore, we think the possible reason for this is that multiple colours cause some disruptive feeling.

In other aspects, even though no significant difference had found, the mean values displayed that, they agreed that they could understand the real-space information such as the shapes, sizes and positions of real objects in all conditions.

\subsubsection{Users' Ability to Perceive the Obstacles in Study 2}

In Study 2, we compared our two 3D interfaces in a scenario when users may collide with obstacles placed inside the playing area. We intended to investigate the usability of our proposed interfaces in this situation and to find out whether adding colour clues based on depth values to the depth sensing interface can improve interaction safety more than the monochrome version. We did not use the 2D interface as a control, since it cannot show up to alert users in this situation. During the experiment, participants were asked to walk as closely as they can to real objects and avoid collisions with the assistance of the VSIs.

Results of understanding of the physical layout information questionnaire demonstrated that subjects' abilities to understand the distances to obstacles, and their capacity for avoiding obstacles were significantly different between two 3D interfaces. Since the mean values of the 3DVCG were all greater than those of the 3DVMG, it is reasonable to say that the 3DVCG had provided more distance clues than the 3DVMG and improved subjects' capacity for avoiding obstacles. Based on the qualitative data, we think the reason behind this can be subjects cannot estimate the distances to obstacles very precisely (in Centimetre) when they walk too close to objects in 3DVMG condition, since the warning information provided in this version is not as intense as that given by the multicolour one. On the contrary, the colour clues assist participants in judging which object is dangerous and which is not, and distances to obstacles from colours. As a result, subjects can make better decisions.

No significant difference was found in terms of the closest distances. We think since, in this test, participants were required to maintain very close distances to obstacles in both conditions, it is unsurprising to find similar behaviours.

Nevertheless, a significant difference was found from the results of the collision count, and the mean values indicated that with the guide of 3DVCG, subjects did bump into real objects less frequently than they did in the other condition. Since there is no significant difference discovered in the closest distance, this aspect does not influence subjects' different behaviours in collision avoidance. Additionally, two 3D interfaces are constructed in the same way, except their colours. Therefore, we think the affecting factor for this phenomenon can only be attributed to the colour effect. Even though subjects stand in similar positions in two conditions, the colour visualization offers them stronger warning information, which results in improving the participants' ability to perceive real objects.

In terms of subjects' preference of two tested interfaces, the most common ranking order was 3DVCG-3DVMG (from the best to the worst), which is as the same as our expectation. We think it is a reasonable outcome 
according to the results of the measurements including physical layout information questionnaire and collision count. Therefore, compared with the 3DVMG, users' ability to perceive the obstacles has been promoted under the help of the coloured grids.

\subsubsection{Comparison of The Usability of Two 3D Interfaces}

In our projects, we investigated the usability of our two proposed 3D interfaces in preventing the occurrence of collisions in two situations: 1) a typical VR room-scale mode, in which obstacles are places outside boundaries of playing areas. Users completed navigating tasks inside the boundaries; 2 ) a new scenario that the currently existing safety interfaces cannot appear to remind users when they are about to collide with obstacles positioned inside the playing area. Users were asked to navigate the virtual environment inside the boundaries as well. The tested 3D interfaces were implemented in the same way, except they presented different obstacles in two studies and they were used in different situations.

By comparing the mean values obtained in the understanding of the physical layout information questionnaire of two 3D interfaces in both studies, we found that the results were very similar for all questions. It means that in both studies, these two 3D interfaces provided a similar experience to subjects regardless of the positions of obstacles (inside or outside the boundaries) and the distances they keep between themselves and real objects during the navigation process (faraway or close). Apart from that, we obtained the same order (3DVCG-3DVMG) from subjects' answers of preference questionnaires in two studies, as expected. Many subjects reported a distinct advantage of multicolours in helping them to predict distances, so we think this is the reason for their answers. Average collision counts of our two 3D interfaces in Study 1 are lower than 0.09, which means that these two 3D interfaces can successfully prevent subjects from colliding with obstacles in a traditional VR roomscale situation. The average collision counts obtained in Study 2 are lower than 1.3, which is higher than the results of Study 1 . We think it is acceptable because distances between themselves and obstacles are extremely short in the second study. We do not expect the two 3D interfaces can have the same performance and maintain an extremely low mean values in Study 2 as they can in Study 1, since it is more difficult for users to avoid obstacles in this case. Therefore, in general, our two 3D interfaces can help users to avoid the collision in both conditions.

Another point we want to discuss is the available space for users to use while applying these two 3D interfaces. As mentioned in section 6.1.1, users keep further distances between themselves and obstacles in two 3D conditions in Study 1, which results in the reduction of the usable space the extent to which. However, the mean values of the item regarding users' confidence in navigating the VE of two 3D interfaces in both studies are similar, which means that even though they stayed in closed positions to obstacles in Study 2, they could still navigate the virtual world confidently. In addition, from the observation of users' behaviours in Study 2, we found a benefit that users can obtain from the 3D interfaces is they can use the space above real objects according to the real-space information provided by the 3D interfaces. For example, some subjects tried to interact with space above a table, while some of them tried to step over the chair to complete their tasks.

Overall, we can draw a conclusion that the usability of our proposed 3D interfaces in helping users to perceive obstacles are generally similar in both scenarios regardless of the positions of obstacles (inside or outside the boundaries) and the distances they keep between themselves and real objects during the navigation process (faraway or close). 


\subsection{Answers to The Proposed Hypotheses}

Based on the data mentioned in the Chapter 5, answers of these two null hypotheses of this study can be drawn as the followings:

- Hypothesis $1(\mathrm{H} 1)$ : There is no significant difference for users to perceive obstacles in the real world between with and without depth information interfaces.

The answer to this hypothesis can be extracted from the results of Study 1 . Significant differences between 2D method and two 3D interfaces respectively were confirmed in three aspects, NOT afraid of suddenly colliding with physical obstacles, collision count and the closest distances. Hence $\mathrm{H} 1$ can be rejected from these aspects.

- Hypothesis $2(\mathrm{H} 2)$ : There is no significant difference in users' ability to perceive obstacles in the real world between with or without colours visualization interfaces.

The answer to this hypothesis can be extracted from the results of Study 2. Significant differences between two 3D interfaces were confirmed in three aspects, subjects' abilities to understand the distances to obstacles, subjects' capacity for avoiding obstacles, and the collision count. Hence $\mathrm{H} 2$ can be rejected from these aspects.

\subsection{Answers to The Proposed Research Questions}

From the results described above, answers of two research questions of our study can be drawn as the followings:

1. Can depth-based virtual safety interfaces improve the interaction safety in VR environments under for real walking navigation?

The answer is with superimposing depth information, two 3D interfaces can improve the interaction safety, because they can assist users to avoid collisions more successfully and reduce their fear of involving with obstacles in a walking-compatible circumstance.

2. Can colour visualization based on depth value improve the interaction safety more?

The answer is compared with appending depth sensing only, the colour visualization can improve the interaction safety better. As participants' abilities to predict distances to obstacles are enhanced, and they can avoid obstacles more precisely in 3DVCG condition.

\subsection{Limitations}

The limitations of our study based on the feedback and the results of the experiment are described as the followings:

- Needing more accuracy of detail of rendered shapes

In study 2, when subjects were asked to walk closely to real objects, the accuracy of drawn grids concerned them. It is understandable as grids are generated based on pictures of the real environment captured by the front camera and the shapes of objects was built with triangle shape. Thus, some pieces of the edge of real objects may not be illustrated entirely accurately because of the depth accuracy. For example, a rightangle shape may be drawn like a round shape in our 3D interfaces, as shown in Figure 24. As a consequence, 
when the distances between users and obstacles are extremely short, such as less than $10 \mathrm{~cm}$, which may lead to misjudging the distance. To address this issue, we can adjust the current geometry drawing method to increase the accuracy of each piece of grids. Nevertheless, considering that in general VR playing circumstance, VR players will not stay in such dangerous positions for a long time. This may not be a large requirement.

- Reduction of the available real space

The data of closes distances in Study 1 indicated that subjects maintained further distances to the obstacles while experiencing two 3D safety interfaces. In terms of addressing safety issues, it is the desired result, but which results in less available real space on the other hand. Limiting users in a small playing area is not what we try to achieve, and actually, we want to make full use of the real space to the contrary. From the results of observation of subjects' behaviour in Study 2, it was found that users could still avoid obstacles successfully even though they were asked to walk as close as they can to the real objects. We can assume that if users can be provided more opportunities to experience the interfaces, their abilities to make use of real space will be improved as well.

- $\quad$ Lack of Real-time Effects

In our study, the developed prototypes are only providing static meshes of the surroundings. If the furniture in the room is moved, our proposed interfaces cannot detect that and update the changes immediately. Likewise, if some people or animals burst in the VR playing area, grids will not remind users as well. For this case, the possible approach can be installing another HTC Vive Pro headset in the playing area to scan the real-world information constantly and reflecting the information in the VE in real time for users.

- Reducing the number of displaying real objects in VR

From the feedback received from users in Study 1, we found that completely illustrating the real space, including further away objects which did not endanger users, made them feel the interface was overloaded and kind of messy. This comment was proved by other researchers' studies as well. Their results have shown that in regard of performance and users' sense of presence, presenting selectively presenting reality which users engaged with was the optimal method, no matter in a standing mode [34] or in a walkingcompatible situation [29]. Apart from that, in Study 2 which only displayed the chair and beanbag, participants did not report they unpleasant feelings. Therefore, the future improvement of our VSIs can be minimised the number of superimposed obstacles in VR or make the visualization of the obstacles more adaptive. 


\section{Chapter 7 Conclusion and Future Work}

\subsection{Conclusion}

In this research, we have implemented two novel VR safety interfaces, 3DVMG and 3DVCG. For the former, we integrated the depth information of the real environment into the interface; we added both depth information of the real space and colour visualization based on depth values for the latter interface. Consequently, these interfaces can provide users with more detailed geometrical information of the surroundings and give subjects alerts as long as they step into potentially dangerous zones of physical obstacles, without restriction of locations of those obstacles. To the contrary, the reference group 2DOoS can only be activated when users reach the boundaries of a playing area. Hence, with our design, even though users do not clean up the playing areas, they can still avoid collisions and freely enjoy VR games at their places with the assistance of our proposed interfaces.

We conducted two user studies to evaluate whether VR navigation safety could be improved with our two proposed interfaces in two different situations.

In Study 1, we compared three interfaces in a typical VR room-scale playing scenario, and the 2DOoS was used as a control. The results of Study 1 indicate that by integrating depth information of the real space, participants can navigate the VE with less worry about safety issues as they can perceive obstacle better. This fact is confirmed by the results of the closest distances and collision count measurements. Additionally, participants preferred 3DVCG and 3DVMG over 2DOoS, and 2DOoS had the worst performance in safely guiding people navigating the VE. Overall, compared with the 2D interface, users' ability to perceive the obstacles has been improved with the help of both 3D interfaces.

In Study 2, we compared our two 3D interfaces in a scenario when users may collide with obstacles placed inside the playing area, and subjects were required to walk close to obstacles. The results demonstrated that the colour visualization provided more hints to users for determining distances to real objects, and it also had a positive effect on assisting in avoiding obstructions. In terms of the collision count, with the help of colour clues, subjects collided with obstacles less than they did in the 3DVMG condition. In this study, subjects also preferred 3DVCG over 3DVMG, as more distance and position clues were provided in 3DVCG. Besides, subjects could make avoiding collisions easier in the 3DVCG condition. Therefore, compared with the 3DVMG, users' ability to perceive the obstacles has been promoted under the help of the coloured grids.

Regarding the immersive experience in both studies, the results of IPQ pointed out that all three tested interfaces allowed subjects to have a similar immersive VR experience. Some limitations relating to the disruptive appearance of our designed interfaces have found from the qualitative data, thus we suggested that executing an appropriate reduction of displaying unnecessary real objects in VR and simplifying the rendering style of interfaces, can be a suitable way to improve immersion.

Overall, our designed 3D interfaces can benefit users in navigating the virtual environment more carelessly, and the 3D interface with colour warning outperforms the single colour one. In addition, an extra advantage of our interfaces has been shown in Study 2. The obstacles used to conduct test 2 were all placed inside the playing area, which means our interfaces can display real objects without limitation of their locations, which the currently existing safety interfaces cannot achieve. It is well known that in a typical room scale mode, an unobstructed space exclusively for VR is required. However, it is difficult to provide such an empty space for home users. Thus, our interfaces can be a suitable approach to address the potential safety issues caused by 
obstacles misplaced within the playing areas. As a result, the requirement of a mostly empty space will not be a burden to many VR players, and VR can be more widely used.

\subsection{Future Work}

For future work of our study, there are several possibilities.

As reported, many VR players sit on their cats ${ }^{17}$ or accidentally punch people around them ${ }^{18}$ when they are playing VR games. Therefore, our first goal it to combine the current pre-scan method with a real-time scanning technique to detect the moving objects around such as people or animals, and update and highlight the prescanned wireframe at particular intervals to represent the change of the environment.

We are also interested in incorporating spatial audio elements to alert users, which has shown its advantage of collision avoidance in VE from a previous study [31]. It is common that the heights of some pieces of furniture at people's rooms are very short. Since we suppose that with our VSIs, people do not need to clean an empty playing area for VR, when the objects are too small or too short to notice, some potential hazards may exist. Therefore, a warning sound may make our interfaces more complete and work more effectively in many situations.

Lastly, many previous studies have focused on the real-world interactions while playing VR in the case where users only stand [35], such as grabbing their drink [33], or under the situation where they can naturally walk in the real space [29]. We are thinking about the possibility in a contrary direction where the player can use the virtual objects to interact with the real objects. It is well acknowledged that a virtual representation of a user in VE could enhance the users' subjective sense of presence in the virtual world [36]. With our developed meshes, we can superimpose the real objects in VE, and then virtual components can be used to interact with the generated meshes, such as placing a virtual object on the meshes. In this case, users can have a sense of touch from real objects represented by the meshes. It can be interesting to investigate the difference of sense of presence between with and without the tactile feedback from the real world.

17 https://venturebeat.com/2017/03/15/this-hack-enables-you-to-see-your-cat-when-youre-in-vr/

${ }^{18}$ https://www.youtube.com/watch?v=rCZPZnwYydo 


\section{Reference List}

[1] Elliot, A. J., Maier, M. A., Moller, A. C., Friedman, R., \& Meinhardt, J. (2007). Colour and psychological functioning: The effect of red on performance attainment. Journal of experimental psychology: General, 136(1), 154.

[2] D.R. Pratt, M. Zyda and K. Kelleher, Virtual reality: In the mind of the beholder, IEEE Computer 28(7) (1995), 17-19.

[3] Suma, E. A., Lipps, Z., Finkelstein, S., Krum, D. M., \& Bolas, M. (2012). Impossible spaces: Maximizing natural walking in virtual environments with self-overlapping architecture. IEEE Transactions on Visualization and Computer Graphics, 18(4), 555-564.

[4] M. Slater. Place illusion and plausibility can lead to realistic behavior in immersive virtual environments. Philosophical Transactions of the Royal Society of London. Series B, Biological Sciences, 364(1535):3549-3457, Dec. 2009.

[5] M. Usoh, K. Arthur, M. C. Whitton, R. Bastos, A. Steed, M. Slater, and F. P. Brooks. Walking $>$ walking-in-place $>$ flying, in virtual environments. In ACM Conference on Computer Graphics and Interactive Tech- niques (SIGGRAPH), pages 359-364, New York, New York, USA, 1999. ACM Press.

[6] S. S. Chance, F. Gaunet, A. C. Beall, and J. M. Loomis. Locomotion Mode Affects the Updating of Objects Encountered During Travel: The Contribution of Vestibular and Proprioceptive Inputs to Path Integration. Presence: Teleoperators and Virtual Environments, 7(2):168-178, Apr. 1998.

[7] R.A. Ruddle and S.Lessels. The benefits of using a walking interface to navigate virtual environments. ACM Transactions on Computer-Human Interaction, 16(1):1-18, Apr. 2009.

[8] Suma, E., Finkelstein, S., Reid, M., Babu, S., Ulinski, A., \& Hodges, L. F. (2010). Evaluation of the cognitive effects of travel technique in complex real and virtual environments. IEEE Transactions on Visualization and Computer Graphics, 16(4), 690-702.

[9] F. Steinicke, G. Bruder, J. Jerald, H. Frenz, and M. Lappe. Estimation of detection thresholds for redirected walking techniques. IEEE Transac- tions on Visualization and Computer Graphics, 16(1):17-27, 2010.

[10] L. Bouguila and M. Sato, “Virtual Locomotion System for Large- Scale Virtual Environment,” Proc. IEEE Virtual Reality Conf., pp. $291-292,2002$.

[11] L. Bouguila, M. Sato, S. Hasegawa, H. Naoki, N. Matsumoto, A. Toyama, J. Ezzine, and D. Maghrebi, "A New Step-in-Place Locomotion Interface for Virtual Environment with Large Display System," Proc. ACM SIGGRAPH, p. 63, 2002

[12] H. Iwata, Y. Hiroaki, and H. Tomioka, “Powered Shoes," Proc. ACM SIGGRAPH, vol. 28, 2006.

[13] H. Iwata, H. Yano, H. Fukushima, and H. Noma, “CirculaFloor,” IEEE Computer Graphics and Applications, vol. 25, no. 1, pp. 64-67, Jan./Feb. 2005.

[14] M. Schwaiger, T. Thu “mmel, and H. Ulbrich, "Cyberwalk: Implementation of a Ball Bearing Platform for Humans," Proc. Int'l Conf. HumanComputer Interaction ( $\mathrm{HCl})$, pp. 926-935, 2007.

[15] J. Jerald, The VR Book: Human-Centered Design for Virtual Reality, Morgan \& Claypool publishersand ACM Books, 2015.

[16] Cirio, G., Marchal, M., Regia-Corte, T., \& Lécuyer, A. (2009, November). The magic barrier tape: a novel metaphor for infinite navigation in virtual worlds with a restricted walking workspace. In Proceedings of the 16th ACM Symposium on Virtual Reality Software and Technology (pp. 155-162). ACM.

[17] Dede, C. (1995). The evolution of constructivist learning environments: Immersion in distributed, virtual worlds. Educational technology, 35(5), 4652.

[18] Dickey, M. D. (2007). Game design and learning: A conjectural analysis of how massively multiple online role-playing games (MMORPGs) foster intrinsic motivation. Educational Technology Research and Development, 55(3), 253-273.

[19] Billinghurst, M., \& Kato, H. (1999, March). Collaborative mixed reality. In Proceedings of the First International Symposium on Mixed Reality (pp. 261-284).

[20] Cohen, J., \& Kupferschmidt, K. (2014). A dose of reality.

[21] Cirio, G., Vangorp, P., Chapoulie, E., Marchal, M., Lécuyer, A., \& Drettakis, G. (2012). Walking in a cube: Novel metaphors for safely navigating large virtual environments in restricted real workspaces. IEEE Transactions on visualization and computer graphics, 18(4), 546-554.

[22] Thomas, B. H. A survey of visual, mixed, and augmented reality gaming. Computers in Entertainment 10, 3 (2012), 3:1-3:33.

[23] RAZZAQUE, S., KOHN, Z., AND WHITTON, M. C. 2001. Redirected walking. In Proceedings of Eurographics.

[24] RUDDLE, R. A., AND LESSELS, S. 2009. The benefits of using a walking interface to navigate virtual environments. ACM Trans. Comput.-Hum. Interact. $16,1,1-18$.

[25] Mehta, R., \& Zhu, R. J. (2009). Blue or red? Exploring the effect of colour on cognitive task performances. Science, 323(5918), 1226-1229.

[26] A. J. Elliot, M. A. Maier, A. C. Moller, R. Friedman, J. Meinhardt, J. Exp. Psychol. Gen. 136, 154 (2007).

[27] Anthony Scavarelli and Robert J. Teather. 2017. VR Collide! Comparing Collision-Avoidance Methods Between Co-located Virtual Reality Users. In Proceedings of the 2017 CHI Conference Extended Abstracts on Human Factors in Computing Systems (CHI EA '17). ACM, New York, NY, USA, 29152921. https://doi.org/10.1145/3027063.3053180

[28] T. Schubert, F. Friedmann, and H. Regenbrecht, "The experience of presence: Factor analytic insights," Presence: Teleoperators and virtual environments, vol. 10, no. 3, pp. 266-281, 2001. 
[29] Kohei Kanamori, Nobuchika Sakata, Tomu Tominaga, Yoshinori Hijikata, Kensuke Harada, and Kiyoshi Kiyokawa. Obstacle avoidance method in real space for virtual reality immersion. In Proceedings of the IEEE International Symposium for Mixed and Augmented Reality 2018 (To appear). 2018.

[30] Afonso, C., \& Beckhaus, S. (2011, May). Collision avoidance in virtual environments through aural spacial awareness. In CHI'11 Extended Abstracts on Human Factors in Computing Systems (pp. 1369-1374). ACM.

[31] Afonso, C., \& Beckhaus, S. (2011, May). Collision avoidance in virtual environments through aural spacial awareness. In CHI'11 Extended Abstracts on Human Factors in Computing Systems (pp. 1369-1374). ACM.

[32] Langbehn, E., Harting, E., \& Steinicke, F. Shadow-Avatars: A Visualization Method to Avoid Collisions of Physically Co-Located Users in Room-Scale VR.

[33] Budhiraja, Pulkit, et al. "Where's My Drink? Enabling Peripheral Real World Interactions While Using HMDs." arXiv preprint arXiv:1502.04744, 2015.

[34] McGill, Mark, et al. "A dose of reality: overcoming usability challenges in vr head-mounted displays." Proceedings of the 33rd Annual ACM Conference on Human Factors in Computing Systems. ACM,pp2143-2152, 2015.

[35] Steinicke, Frank, et al. "Poster: A virtual body for augmented virtuality by chroma-keying of egocentric videos." 3D User Interfaces, 2009. 3DUI 2009. IEEE Symposium on. IEEE,pp125-126, 2009.

[36] G. Bruder, F. Steinicke, K. Rothaus, and K. Hinrichs, "Enhancing presence in head-mounted display environments by visual body feedback using head-mounted cameras," in CyberWorlds, 2009. CW'09. International Conference on. IEEE, 2009, pp. 43-50. 


\section{Appendix A: Information Sheet and Consent Form}

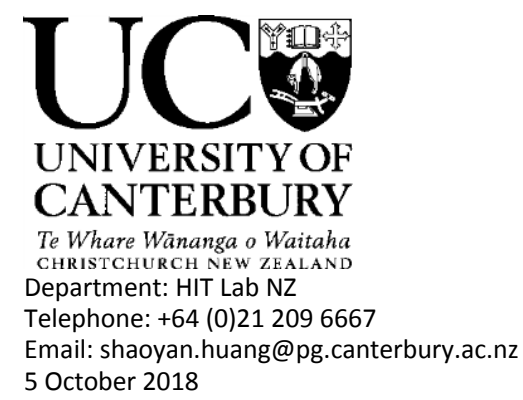

Novel Virtual Reality Safety Precaution Interface Research

Information Sheet for Participants

The goal of this study is to investigate whether VR navigation safety can be improved with depth sensing and colour warning information when walking navigation is used. There are three virtual safety interfaces in this study: 1) 2D outlines of surroundings, 2) 3D Monochrome Grids and 3) 3D Coloured Grids. They are used to warn you about physical obstacles in the real world when you approach them during the process of navigating the virtual environment.

If you choose to take part in this study, your involvement in this project will be navigating a virtual garden by following fixed routes using real walking. There are two studies in this experiment: one is to examine the usability of the three virtual safety interfaces when navigating along the boundary of playing areas; the other one is to test the usability of the latter two interfaces when navigating inside playing areas. There are five tests in total. In each test, you will be exposed to one of those virtual safety interfaces randomly. Once you complete one condition, you can take off the headset and have a break. Each study will last around 2 minutes, and the whole experiment will last about 40 minutes.

Before the experiment, some basic demographic information will be collected (e.g., age group, past experience with VR), but any published results will be anonymous. After each condition is completed, you will be asked to complete a per-condition questionnaire. A post questionnaire will need to be filled out at the end of each study. The procedure will also be video recorded to ensure accuracy. A $\$ 10$ voucher will be given to you at the end. In the performance of the tasks and application of the procedures, there is a risk of dizziness (also known as cybersickness) due to the use of the VR headset. You can stop the experiment at any time or extend the period between sessions to as long as you need. We will also offer a couch where you can relax until the symptoms have faded.

During the experiment, there is also a risk of colliding with obstacles in the real environment, for example when you get close to the edge of the play area. To prevent and mitigate this risk, the furniture used in the experiment room will be soft chairs or sofas, or be covered with soft cloth, which will not harm you. Furthermore, during the experiment the researcher will always be at a distance of intervention to prevent any incident. If at any time during the experiment the experimenter detects any problem that could endanger you, you will be notified, and the experiment will be paused until the danger is removed.

Participation is voluntary and you have the right to withdraw at any stage without penalty. You may ask for your raw data to be returned to you or destroyed at any point. If you withdraw, any information relating to you will be removed, however, once analysis of raw data starts on 20 December 2018, it will become increasingly difficult to remove the influence of your data on the results.

The results of the project may be published for future use beyond the master thesis, but you may be assured of the complete confidentiality of data gathered in this investigation: your identity will not be made public without your prior consent. To ensure anonymity and confidentiality all the data will be stored securely and only the researchers mentioned on the consent form will have access to it. However, we might also share parts of the raw anonymized data with other researchers if there is a need to do so. The data will be kept securely stored 
for a minimum period of five years on storage systems within the University of Canterbury, and securely destroyed after that.

Please indicate to the researcher on the consent form if you would like to receive a copy of the summary of results of the project.

The project is being carried out by Shaoyan Huang (shaoyan.huang@pg.canterbury.ac.nz) under the supervision of Rob Lindeman and Huidong Bai, who can be contacted at gogo@hitlabnz.org and huidong.bai@auckland.ac.nz respectively. They will be pleased to discuss any concerns you may have about participation in the project.

This project has been reviewed and approved by the University of Canterbury Human Ethics Committee, and participants should address any complaints to The Chair, Human Ethics Committee, University of Canterbury, Private Bag 4800, Christchurch (human-ethics@canterbury.ac.nz).

If you agree to participate in the study, you are asked to complete the consent form and give it to the test moderator. 
Email: shaoyan.huang@pg.canterbury.ac.nz

5 October 2018

\section{Virtual Reality Safety Interface Research}

\section{Consent Form for Participants}

I have been given a full explanation of this project and have had the opportunity to ask questions.

I understand what is required of me if I agree to take part in the research.

I understand that participation is voluntary, and I may withdraw at any time without penalty. Withdrawal of participation will also include the withdrawal of any information I have provided should this remain practically achievable.

I understand that any information or opinions I provide will be kept confidential to the researcher Shaoyan Huang and the supervisors Rob Lindeman and Huidong Bai, and that any published or reported results will not identify me. I understand that a thesis is a public document and will be available through the UC Library.

I understand that all data collected for the study will be kept in locked and secure facilities and/orin password protected electronic form and will be destroyed after five years.

I understand that parts of the anonymized data could be shared with other researchers beyond this research if there is a need to do so in the future (e.g., related development, teaching or research).

$\square$ I understand the risks associated with taking part and how they will be managed.

$\square$ I understand that I can contact the researcher [Shaoyan Huang, shaoyan.huang@pg.canterbury.ac.nz] or supervisors [Professor Rob Lindeman, gogo@ hitlabnz.org, +64 3369 2436], [Huidong.Bai, huidong.bai@ auckland.ac.nz ] for further information. If I have any complaints, I can contact the Chair of the University of Canterbury Human Ethics Committee, Private Bag 4800, Christchurch (human-ethics@canterbury.ac.nz)

I would like a summary of the results of the project.

By signing below, I agree to participate in this research project.

Name:__Signed:__Date:

Email address (for report of findings, if applicable): 


\section{Appendix B: Questionnaires}

\section{Demographic questions}

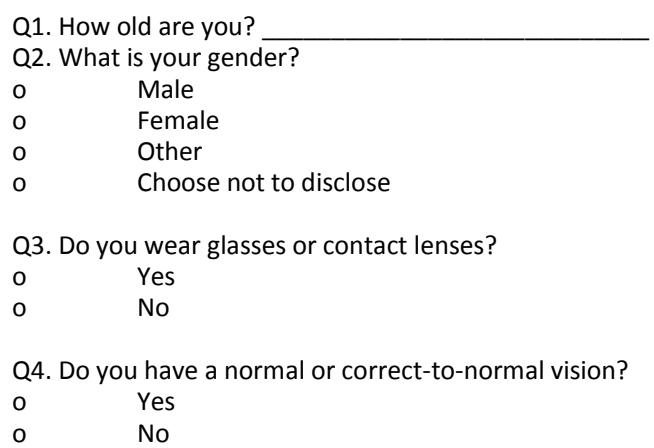

Q5. Have you used VR headsets before? (For example, Oculus Rift, HTC Vive or Samsung Gear VR)

$\begin{array}{ll}\text { o } & \text { Never } \\ \text { O } & \text { Once } \\ \circ & \text { Once a month } \\ \circ & \text { Once a week }\end{array}$

Daily

Q6. Which VR headsets have you used before? Tick all that apply

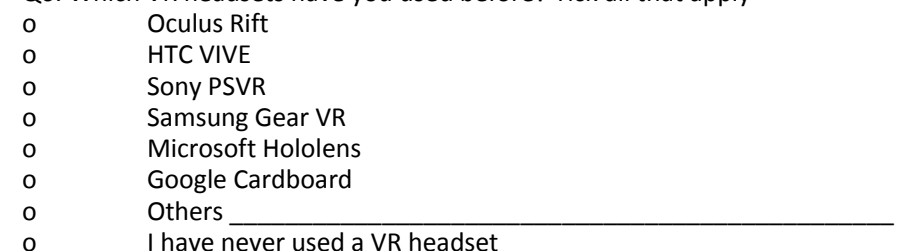

Q7. Have you ever bumped into obstacles, such as tables or chairs, in the real world while you are wearing a VR headset and exploring the virtual environment?

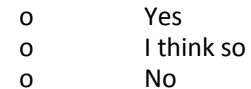

Q8. Have you ever seen the virtual grid wall similar to the picture below in your virtual reality experience?

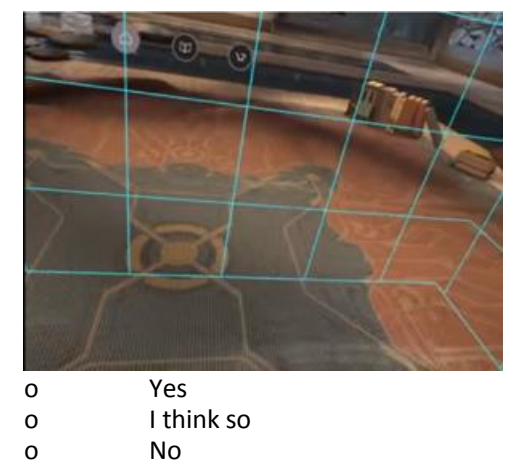




\section{Per-Condition Questionnaire -Igroup Presence Questionnaire}

Q1. In the computer-generated garden, I had a sense of "being there".

Strongly disagree

Disagree

Somewhat disagree

Neither agree nor disagree

Somewhat agree

Agree

Strongly agree

Q2. Somehow I felt that the virtual world surrounded me.

o Strongly disagree

Disagree

Somewhat disagree

Neither agree nor disagree

Somewhat agree

Agree

Strongly agree

Q3. I felt like I was just perceiving pictures.

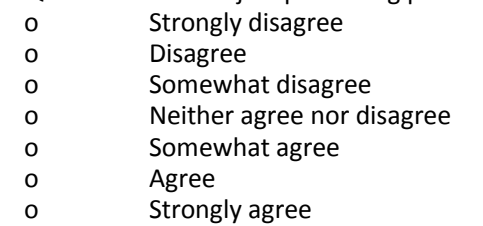

Q4. I did not feel present in the virtual space.

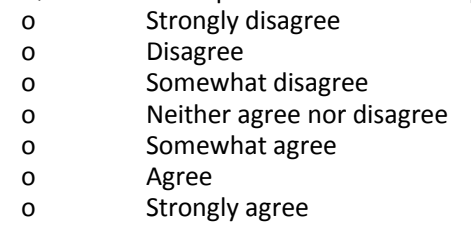

Q5. I had a sense of acting in the virtual space, rather than operating something from outside.

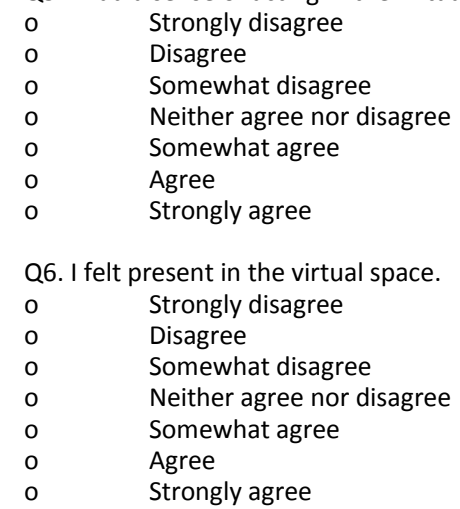

Q7. I was aware of the real world surrounding while navigating in the virtual world. (i,e, sounds, room temperature, other people, etc.)

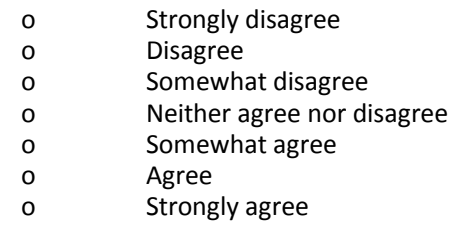

Q8. I was NOT aware of my real environment.

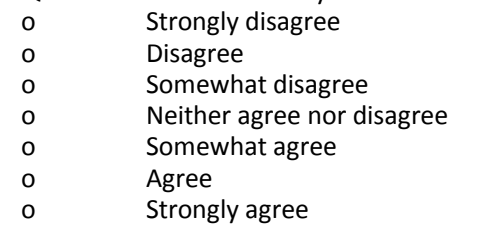

Q9. I still paid attention to the real environment.

o Strongly disagree

o Disagree 
Somewhat disagree

Neither agree nor disagree

Somewhat agree

Agree

Strongly agree

Q10. I was completely captivated by the virtual world.

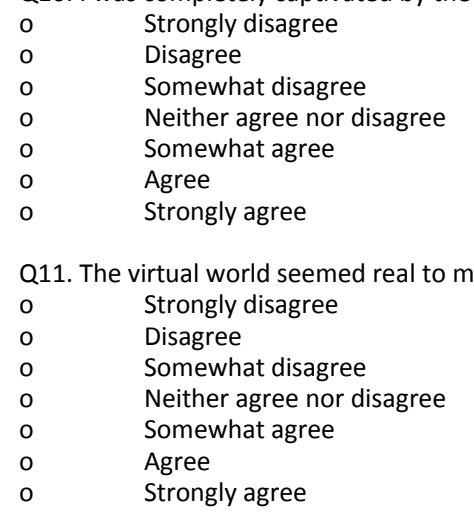

Q12. My experience in the virtual environment seemed consistent with my real-world experience.

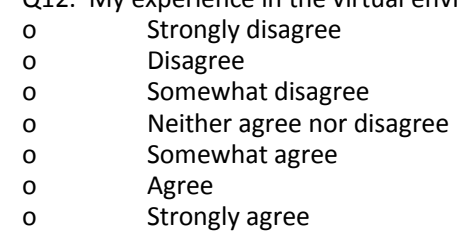

Q13. The virtual world seemed real to me.

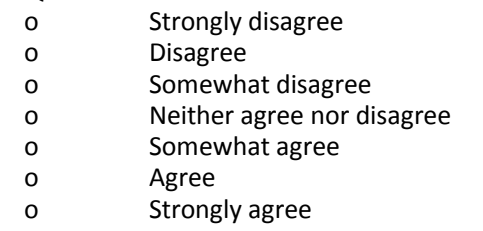

Q14. The virtual world seemed more realistic than the real world.

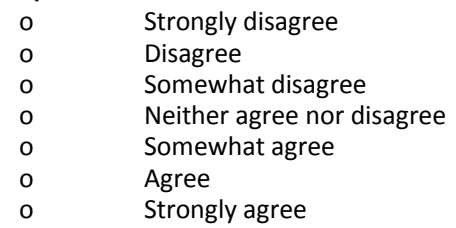

\section{Per-condition: Understanding of physical layout information Questionnaire}

Q15. I was aware of the presence of obstacles in the real world with the help of the virtual safety interface (for example, it seemed that there was a table in front of me).

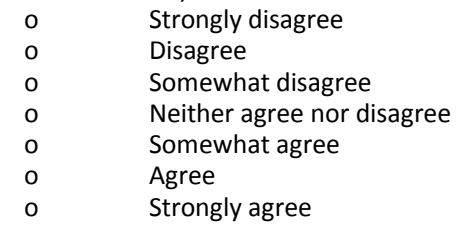

Q16. I could accurately understand the distance to obstacles in the real world with the help of the virtual safety interface.

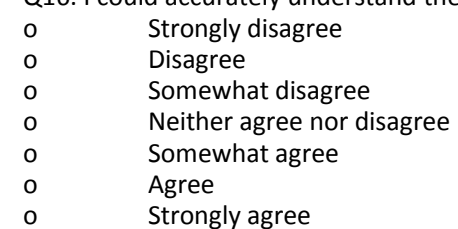

Q17. I could accurately understand the shape of obstacles in the real world with the help of the virtual safety interface.

o Strongly disagree

o Disagree

o Somewhat disagree

o Neither agree nor disagree 
Strongly agree

Q18. I could accurately understand the size of obstacles in the real world with the help of the virtual safety interface.

Strongly disagree

Disagree

Somewhat disagree

Neither agree nor disagree

Somewhat agree

Agree

Strongly agree

Q19. I could understand the viewing direction in the real world with the help of the virtual safety interface. Strongly disagree

Disagree

Somewhat disagree

Neither agree nor disagree

Somewhat agree

Agree

Strongly agree

Q20. I could guess my own position in the real world with the help of the virtual safety interface.

o Strongly disagree

Disagree

Somewhat disagree

Neither agree nor disagree

Somewhat agree

Agree

Strongly agree

Q21. I could precisely predict the location of physical obstacles with the help of the virtual safety interface.

Strongly disagree

Disagree

Somewhat disagree

Neither agree nor disagree

Somewhat agree

Agree

Strongly agree

Q22. I could precisely avoid physical obstacles with the help of the virtual safety interface. Strongly disagree

Disagree

Somewhat disagree

Neither agree nor disagree

Somewhat agree

Agree

Strongly agree

Q23. I was NOT afraid of suddenly colliding with physical obstacles with the help of the virtual safety interface.

o Strongly disagree

Disagree

Somewhat disagree

Neither agree nor disagree

Somewhat agree

Agree

Strongly agree

Q24. I could navigate the virtual garden quickly with the help of the virtual safety interface.

Strongly disagree

Disagree

Somewhat disagree

Neither agree nor disagree

Somewhat agree

Agree

Strongly agree

Q25. I felt the real surroundings were represented very well with the safety interface.

Strongly disagree

Disagree

Somewhat disagree

Neither agree nor disagree

Somewhat agree

Agree

Strongly agree

Q26. I felt the appearance of the virtual safety interface was disruptive to the virtual experience.

o Strongly disagree

o Disagree 
Somewhat disagree

Neither agree nor disagree

Somewhat agree

Agree

Strongly agree

Q27. I felt mentally stressful when I was navigating the virtual garden.

o Strongly disagree

Disagree

Somewhat disagree

Neither agree nor disagree

Somewhat agree

Agree

Strongly agree

\section{Post-experiment questionnaire (Study 1)}

Q1. Rank (1-3) the following interfaces in order of helping to predict the distance to physical obstacles from yourself when navigating the virtual garden. (The most preferred item $=1$ )

2D Outlines of Surroundings

3D Virtual Monochrome Grid

3D Virtual Coloured Grid

Q2. Rank the following interfaces in order of helping to avoid physical obstacles when navigating the virtual garden.

2D Outlines of Surroundings

3D Virtual Monochrome Grid

3D Virtual Coloured Grid

Q3. Rank the following interfaces in order of helping to confidently navigate the virtual garden.

2D Outlines of Surroundings

3D Virtual Monochrome Grid

3D Virtual Coloured Grid

Q4. Overall, rank the following in order of preference for navigating the virtual garden with better safety precaution. 2D Outlines of Surroundings

3D Virtual Monochrome Grid

3D Virtual Coloured Grid

Q5. What are the reasons that you collided with physical obstacles during the experiment? (Only explain if you had. If not, just write None.)

2D Outlines of Surroundings
3D Virtual Monochrome Grid

3D Virtual Coloured Grid

Q6. What are the advantages of using one interface compared with the others in terms of assisting avoiding collisions, presence, and cybersickeness?

2D Outlines of Surroundings

3D Virtual Monochrome Grid

3D Virtual Coloured Grid

Q7. What problems are there for each interface in terms of assisting avoiding collisions, presence, and cybersickeness?

- 2D Outlines of Surroundings

3D Virtual Monochrome Grid

o 3D Virtual Coloured Grid

Q8. Any other comments on the experiment?

\section{Post-experiment Questionnaire (Study 2)}

Q1. Rank the following interfaces in order of helping to predict the distance to physical obstacles from yourself when navigating the virtual garden. (The most preferred item at the top)

3D Virtual Monochrome Grid

3D Virtual Coloured Grid

Q2. Rank the following interfaces in order of helping to avoid physical obstacles when navigating the virtual garden.

3D Virtual Monochrome Grid

3D Virtual Coloured Grid

Q3. Rank the following interfaces in order of helping to confidently navigate the virtual garden.

3D Virtual Monochrome Grid

3D Virtual Coloured Grid

Q4. Overall, rank the following in order of preference for navigating the virtual garden with better safety precaution.

3D Virtual Monochrome Grid 
Q5. What are the reasons that you collided with physical obstacles during the experiment? (Only explain if you had. If not, just write None.) o 3D Virtual Monochrome Grid

o 3D Virtual Coloured Grid

Q6. What are the advantages of using one interface compared with the others in terms of assisting avoiding collisions, presence, and cybersickeness? o 3D Virtual Monochrome Grid

o 3D Virtual Coloured Grid

Q7. What problems are there for each interface in terms of assisting avoiding collisions, presence, and cybersickeness?

o 3D Virtual Monochrome Grid

o 3D Virtual Coloured Grid

Q8. Any other comments on the experiment? 